\title{
Continuous Pd-Catalyzed Carbonylative Cyclization using Iron Pentacarbonyl as a CO Source
}

Pavol Lopatka, ${ }^{\dagger}$ Martin Markovič, ${ }^{\dagger}$ Peter Koóšs ${ }^{\dagger}{ }^{\dagger *}$ Steven V. Ley ${ }^{\ddagger}$ and Tibor Gracza ${ }^{\dagger}$

'Department of Organic Chemistry, Institute of Organic Chemistry, Catalysis and Petrochemistry, Slovak University of Technology, Radlinského 9, SK-812 37 Bratislava, Slovakia

Department of Chemistry, University of Cambridge, Lensfield Road, Cambridge CB2 1EW, U.K.

${ }^{\S}$ Georganics Ltd., Koreničova 1, SK-811 03 Bratislava, Slovakia

\section{Supporting information}

\section{Table of contents}

$\begin{array}{ll}\text { Synthesis of starting materials } & \text { S2 }\end{array}$

Table S1. Optimization of reaction conditions $\quad$ S7

Figures of flow system setup for carbonylation reactions $\quad$ S8

$\begin{array}{ll}\text { References } & \text { S10 }\end{array}$

Copies of ${ }^{1} \mathrm{H}$ and ${ }^{13} \mathrm{C}$ NMR spectra $\quad \mathrm{S} 11$ 


\section{Synthesis of starting materials}

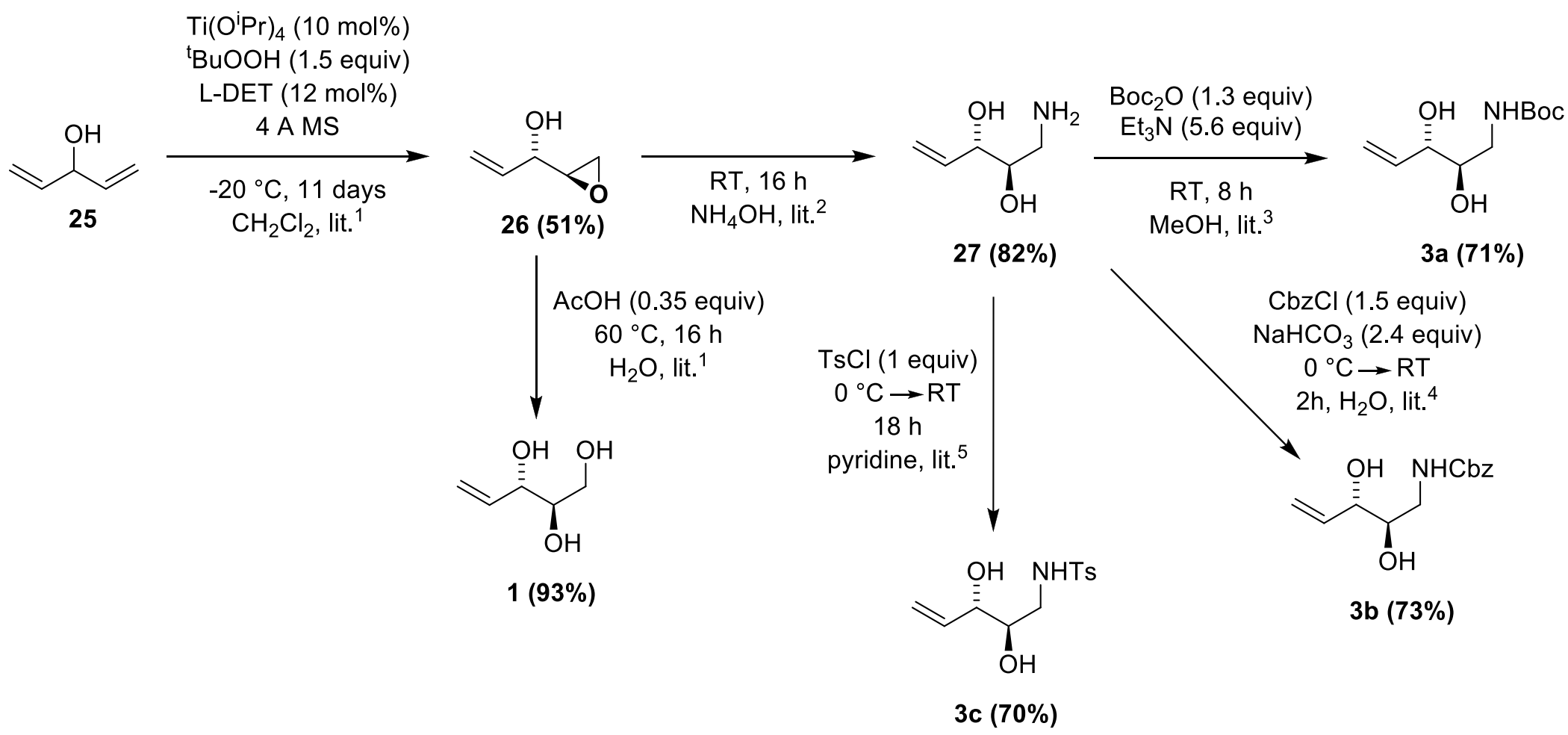

Scheme S1: Synthesis of optically pure unsaturated polyols/aminoalcohols. 


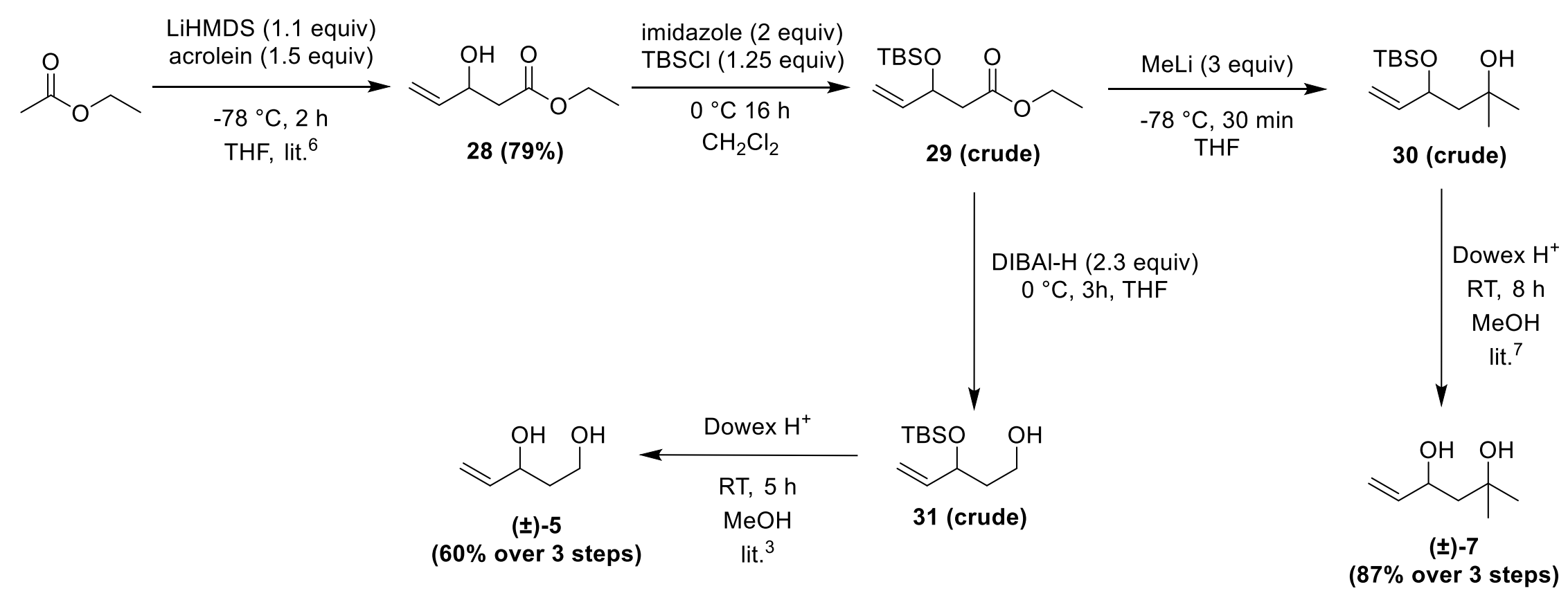

Scheme S2: Synthesis of $\mathrm{C}_{5}$ unsaturated diols. 


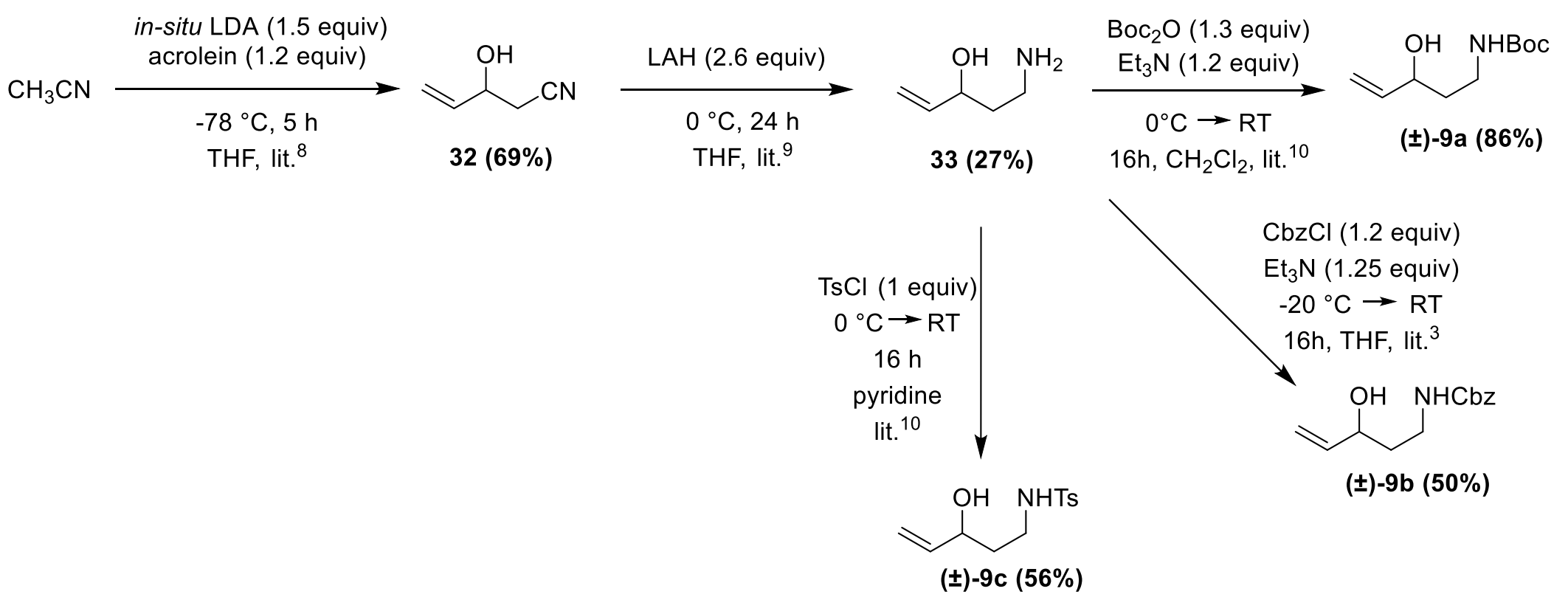

Scheme S3: Synthesis of various $C_{5}$ aminoalcohols. 


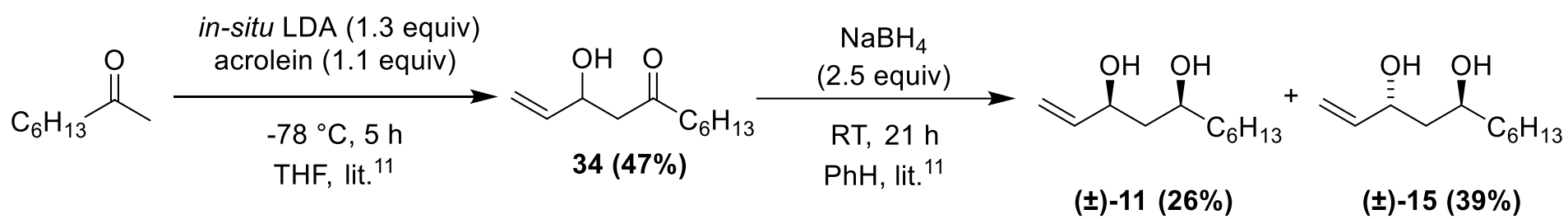

$\mathrm{NaBH}(\mathrm{OAc})_{3}$ (5 equiv)

$\mathrm{RT}, 18 \mathrm{~h}, \mathrm{PhH}$

lit. 11

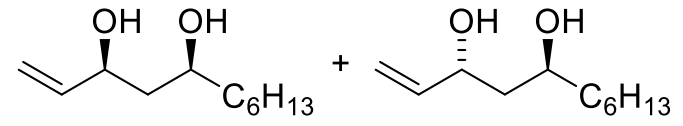

$( \pm)-11(17 \%) \quad( \pm)-15(52 \%)$

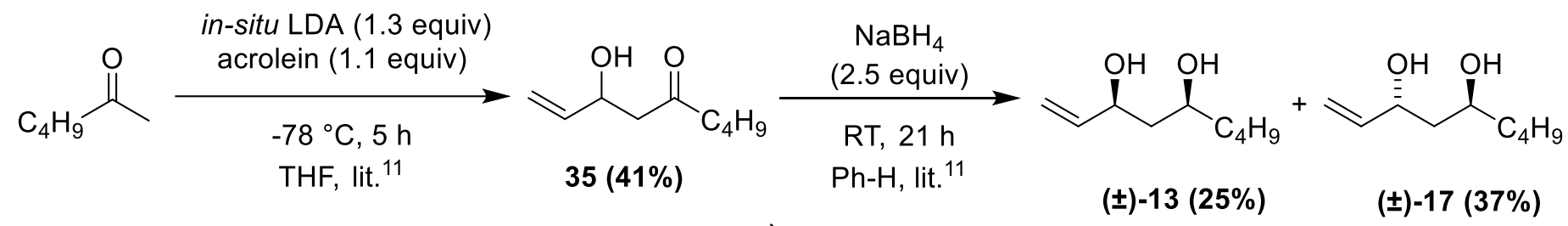

$\mathrm{NaBH}(\mathrm{OAc})_{3}$ (5 equiv)

$\mathrm{RT}, 18 \mathrm{~h}, \mathrm{Ph}-\mathrm{H}$

lit. $^{11}$

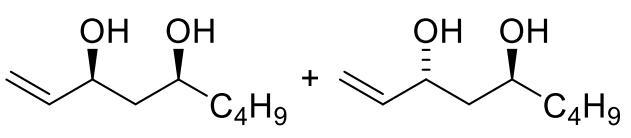

$( \pm)-13(18 \%) \quad( \pm)-17(54 \%)$

Scheme S4: Synthesis of various $C_{9}$ and $C_{11}$ unsaturated diols. 

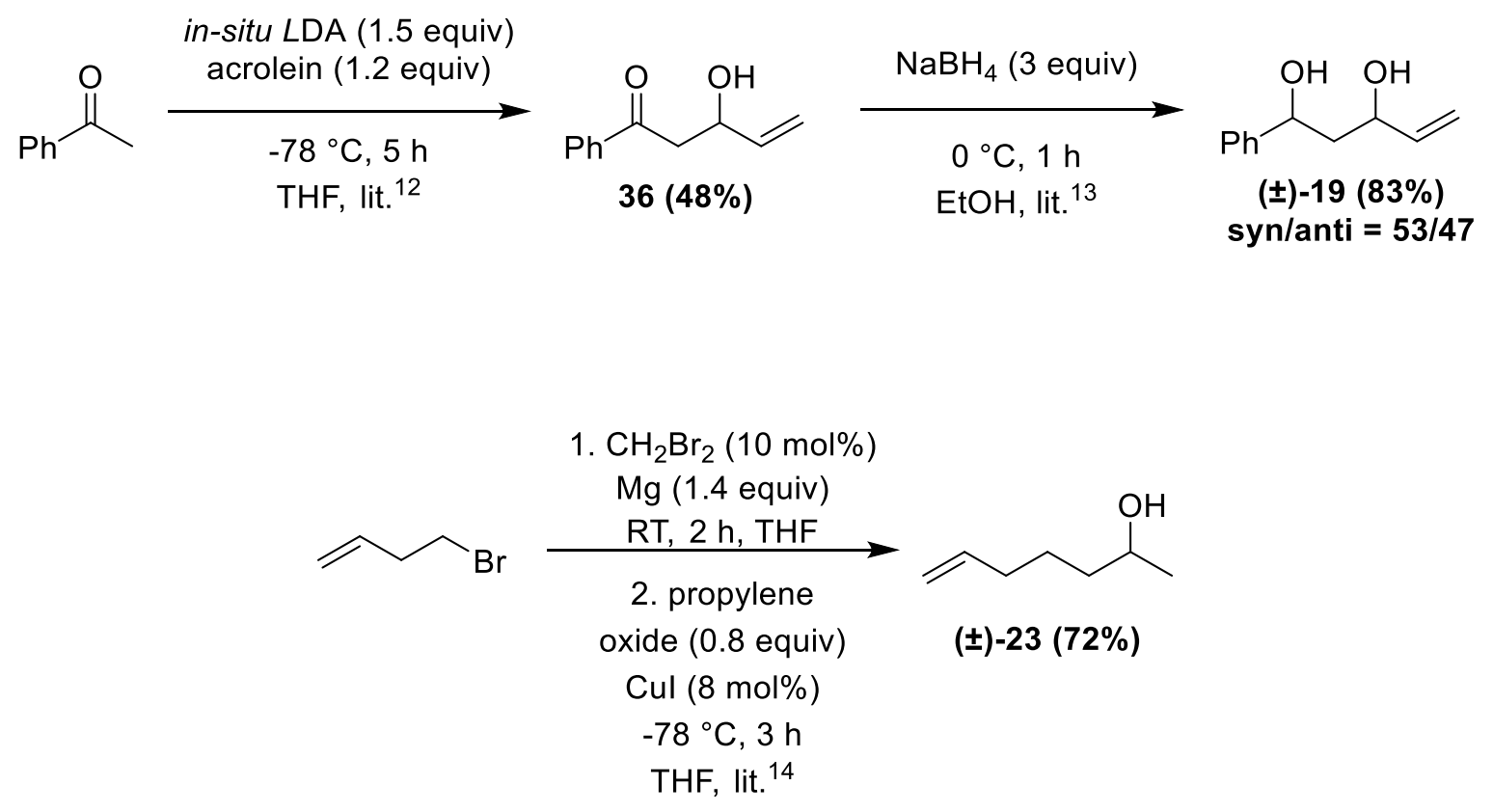

Scheme S5: Synthesis of substrates ( \pm )-19 and ( \pm )-23. 


\section{Table S1. Optimization of reaction conditions}

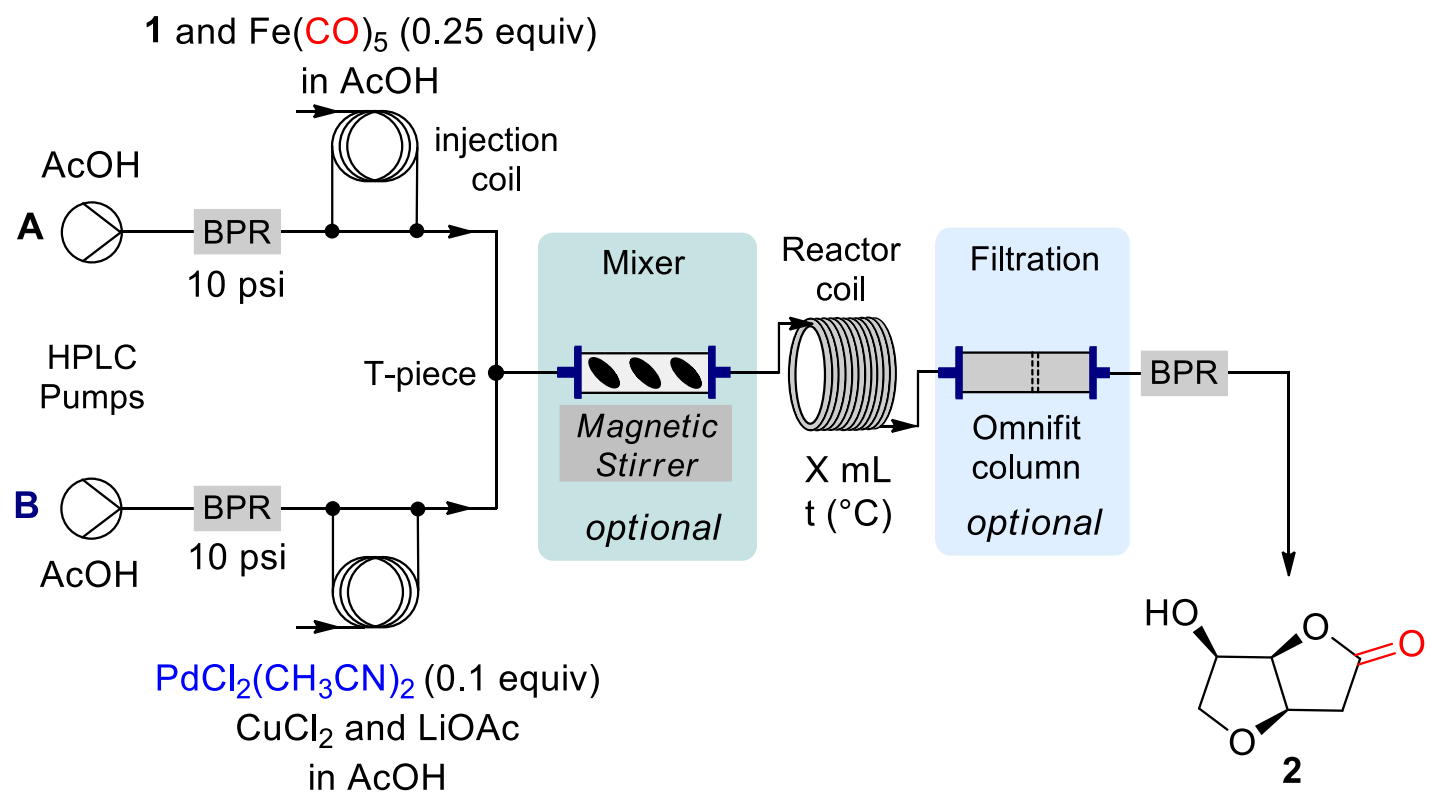

Flow setup: 1/8 inch PTFE tubing, Knauer HPLC pumps, injection coil A was loaded with substrate = pent-1-en3,4,5-triol (scale) and $\mathrm{Fe}(\mathrm{CO})_{5}$ in $\mathrm{AcOH}$, injection coil B was loaded with $\mathrm{PdCl}_{2}\left(\mathrm{CH}_{3} \mathrm{CN}\right)_{2}(0.1$ eq.) and reoxidation system $\left(\mathrm{CuCl}_{2} / \mathrm{LiOAc}=1 / 2\right)$,

\begin{tabular}{|c|c|c|c|c|c|c|c|c|c|c|c|}
\hline Entry & $\begin{array}{c}\text { Reox. } \\
\text { system }^{\mathrm{a}}\end{array}$ & $\begin{array}{c}\mathrm{CO} \\
(\mathbf{e q} .)^{\mathrm{b}}\end{array}$ & $\begin{array}{c}\text { Scale } \\
\left(\mathrm{mmol} / \mathbf{M}^{\mathrm{c}}\right)\end{array}$ & $\begin{array}{c}\text { Flow } \\
\text { Rate A/B } \\
\text { (mL/min) }\end{array}$ & $\begin{array}{c}\text { Rxn } \\
\text { coil } \\
(\mathbf{m L})\end{array}$ & $\begin{array}{c}\text { Rxn } \\
\text { time } \\
\text { (min) } \\
\end{array}$ & $\begin{array}{c}\mathbf{t} \\
\left({ }^{\circ} \mathbf{C}\right)\end{array}$ & Mixer & $\underset{d}{\text { Filtr. }}$ & $\begin{array}{l}\text { BPR } \\
\text { (psi) }\end{array}$ & $\begin{array}{c}\text { Yield } \\
(\%)\end{array}$ \\
\hline 1 & 4 & 1.25 & $0.3 / 0.4$ & $0.08 / 0.55$ & 6 & 10 & 60 & - & - & 100 & - \\
\hline 2 & 3 & 1.25 & $0.4 / 0.6$ & $0.08 / 0.55$ & 6 & 10 & 60 & - & - & 100 & 33 \\
\hline 3 & 2.5 & 1.25 & $0.5 / 0.7$ & $0.08 / 0.55$ & 6 & 10 & 60 & - & - & 100 & 55 \\
\hline 4 & 3 & 1.25 & $0.4 / 0.6$ & $0.08 / 0.55$ & 6 & 10 & 40 & - & - & 100 & 32 \\
\hline 5 & 3 & 1.25 & $0.4 / 0.6$ & $0.05 / 0.35$ & 9 & 20 & r.t. & - & - & - & 20 \\
\hline 6 & 3 & 1.25 & $1.8 / 0.6$ & $0.1 / 0.7$ & 8 & 10 & 60 & + & - & - & 47 \\
\hline 7 & 3 & 1.25 & $1.8 / 0.6$ & $0.07 / 0.47$ & 8 & 15 & 60 & + & - & - & 56 \\
\hline 8 & 3 & 1.25 & $2.2 / 0.6$ & $0.11 / 0.75$ & 26 & 30 & 60 & + & - & - & 58 \\
\hline 9 & 3 & 1.25 & $2.2 / 0.6$ & $0.22 / 1.5$ & 26 & 15 & 60 & + & + & 15 & 56 \\
\hline 10 & 3.5 & 1.25 & $1.9 / 0.5$ & $0.22 / 1.5$ & 26 & 15 & 60 & + & + & 15 & 59 \\
\hline 11 & 3.5 & 1.5 & $1.9 / 0.5$ & $0.22 / 1.5$ & 26 & 15 & 60 & + & + & 15 & 63 \\
\hline
\end{tabular}

${ }^{a} \mathrm{X}$ eq. of reox. system corresponds to $\mathrm{X}$ eq. of $\mathrm{CuCl}_{2}$ and $2 \mathrm{X}$ eq.of $\mathrm{LiOAc}\left(0.25 \mathrm{M}\right.$ solution of $\mathrm{CuCl}_{2}$ in $\mathrm{AcOH}$, coil B)

b 1.25 eq. of $\mathrm{CO}$ corresponds to 0.25 eq. of $\mathrm{Fe}(\mathrm{CO})_{5}$

${ }^{\mathrm{c}}$ concentration of substrate in $\mathrm{AcOH}$ (coil A)

${ }^{\mathrm{d}}$ HPLC column with frit was used as a filtration device 
Flow system setup for carbonylation reactions

Figure S1. Flow system for method 1

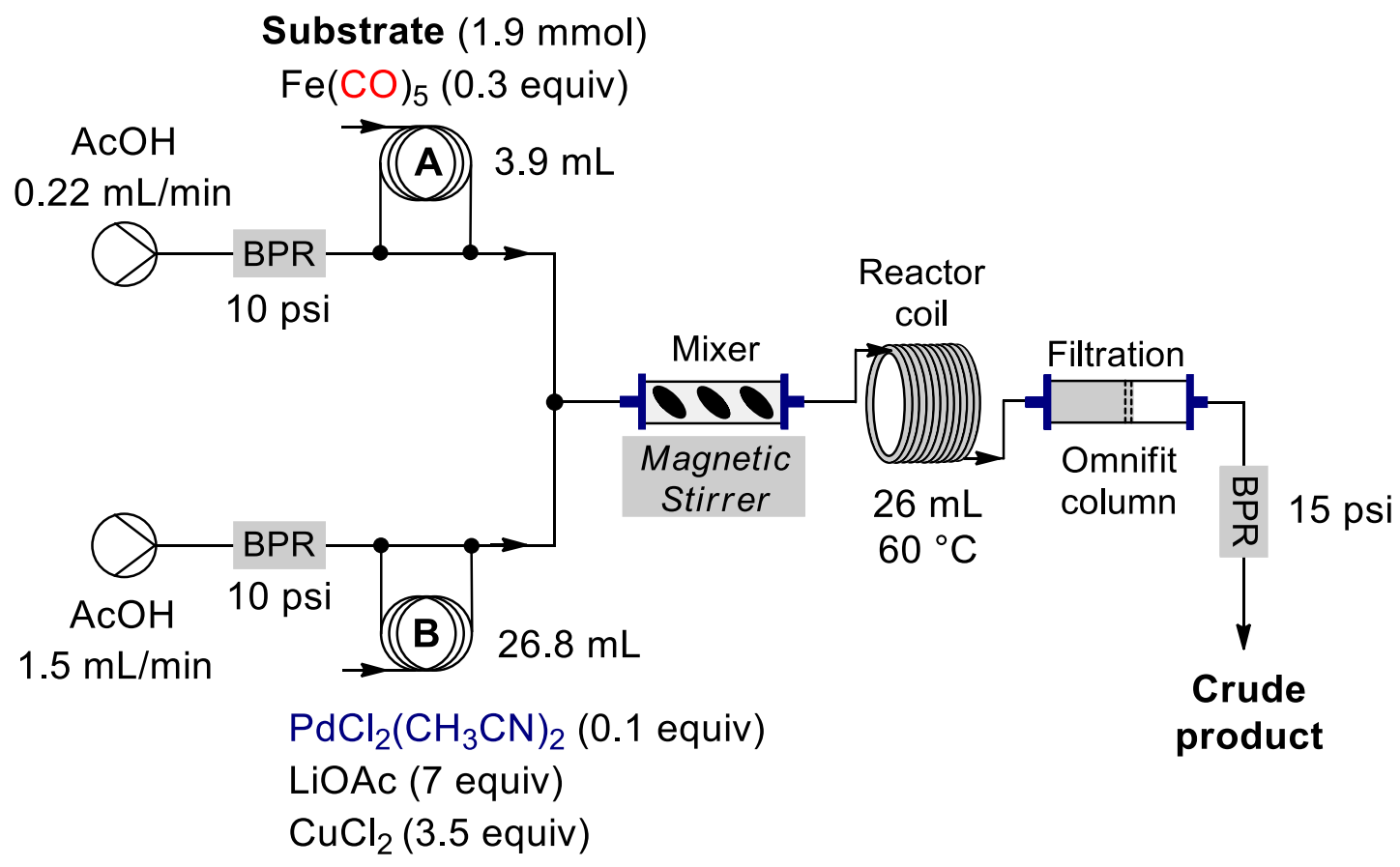

Figure S2. Flow system for method 2

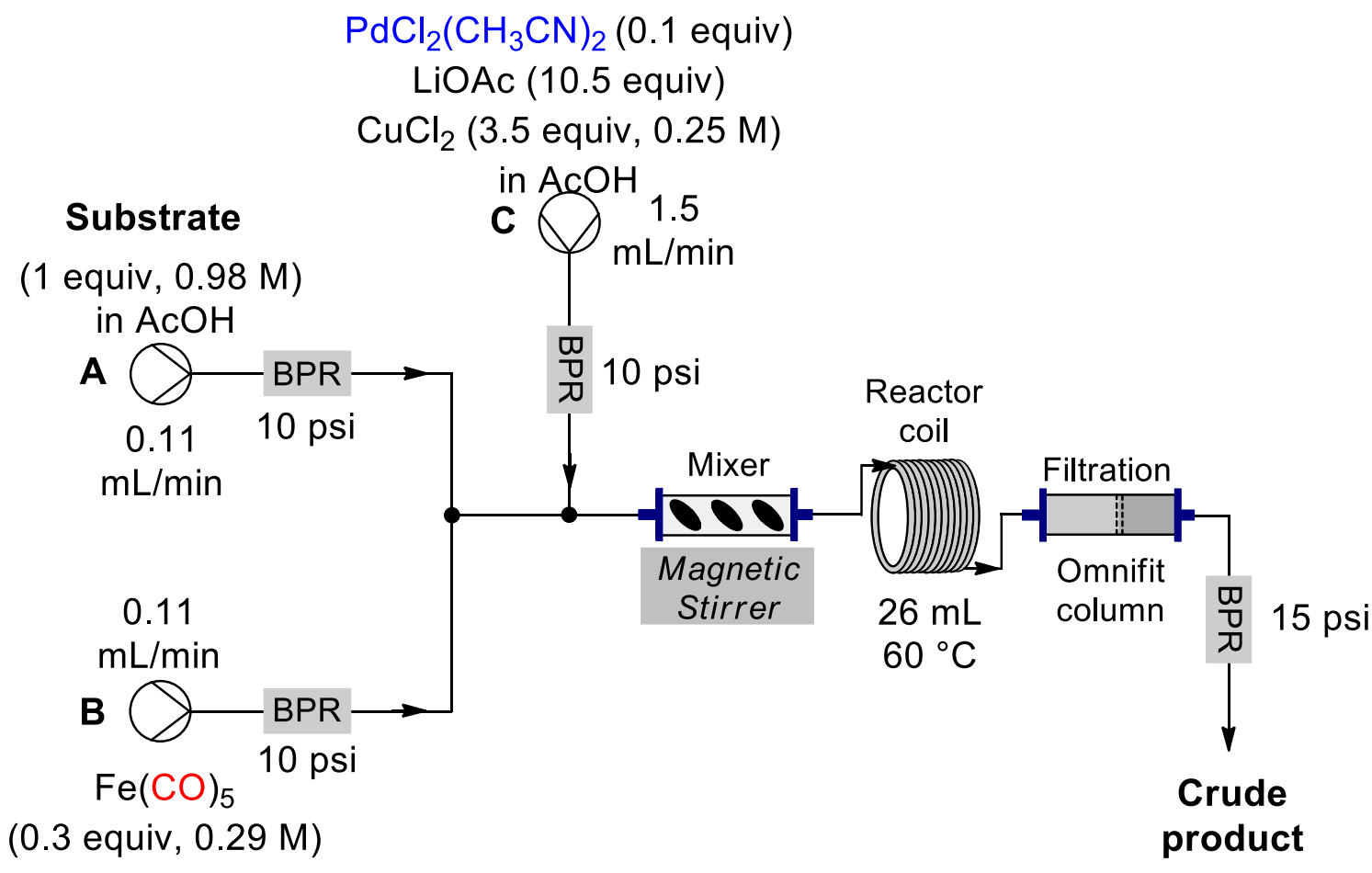


Figure S3. Flow system for method 3

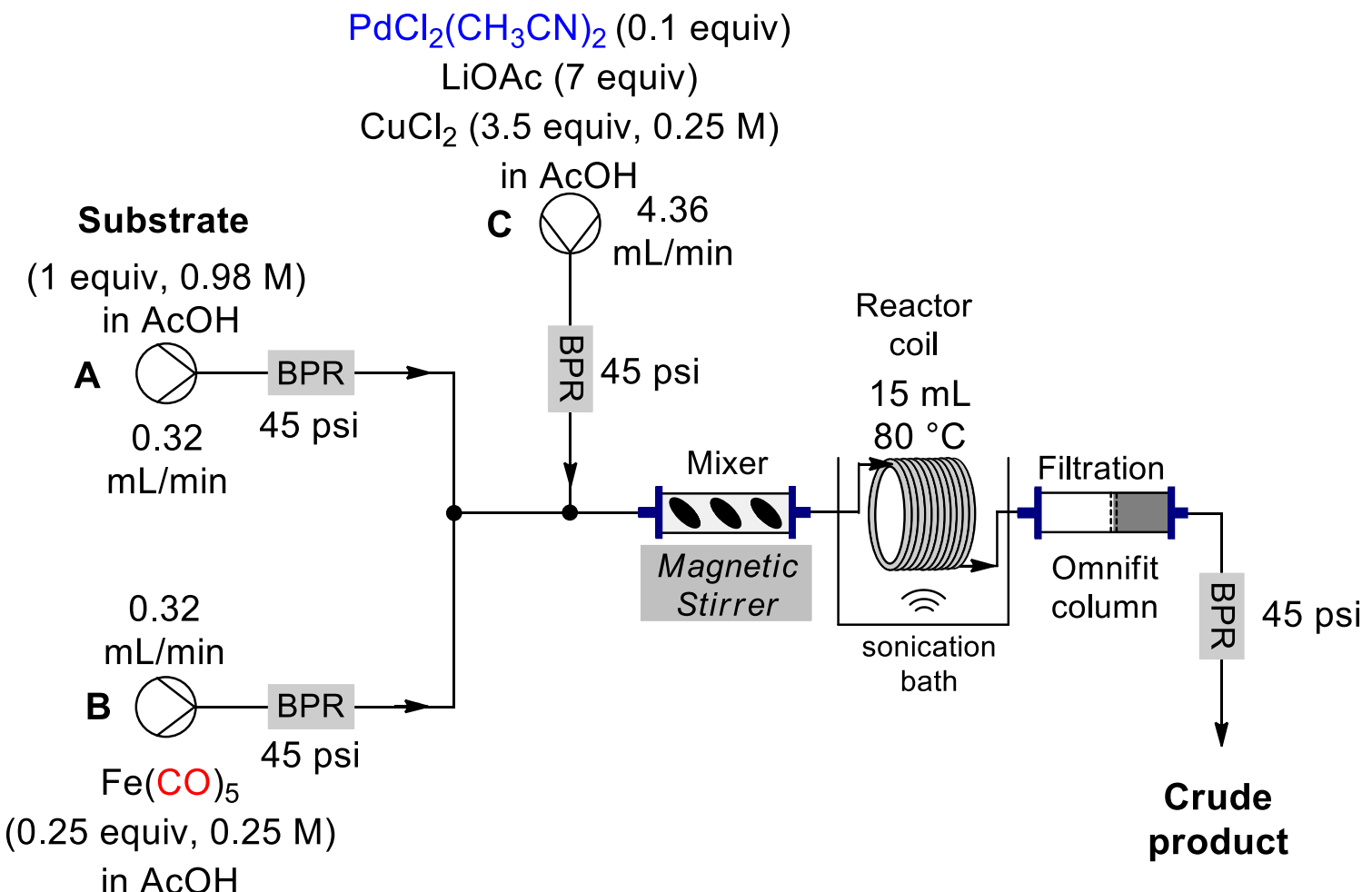




\section{References}

1. Jäger, V., Schröter, D., Koppenhoefer, B. Asymmetric sharpless epoxidation of divinylcarbinol. Erythro-D- and -L-4-pentenitols by hydrolysis of regioisomeric epoxy-4pentenols. Tetrahedron 1991, 47, 2195-2210.

2. Hümmer, W., Gracza, T., Jäger, V. Regiocontrol in the synthesis of optically active amino4-pentenediols via epoxy-4-pentenols. Novel acyclic adenosine analogues. Tetrahedron Lett. 1989, 30, 1517-1520.

3. Babjak, M., Markovič, M., Kandríková, B., Gracza, T. Homogeneous Cyclocarbonylation of Alkenols with Iron Pentacarbonyl. Synthesis 2014, 46, 809-816.

4. Jäger, V., Hümmer, W., Stahl, U., Gracza, T. Controlled Synthesis of Enantio-, Regio-, and Diastereomers of Amino-4-pentenediols from 1,4-Pentadien-3-ol via Epoxy-4-pentenols I. erythro-1-Amino-4-pentene-2,3-diols. Synthesis 1991, 769-776.

5. Hümmer, W., Dubois, E., Gracza, T., Jäger, V. Halocyclization and Palladium(II)-Catalyzed Amidocarbonylation of Unsaturated Aminopolypols. Synthesis of 1,4-Iminoglycitols as Potential Glycosidase Inhibitors. Synthesis 1997, 634-642.

6. Crimmins, M. T., King, B. W., Watson, P. S., Guise, L. E. Synthesis and intramolecular photocycloadditions of 2-acyloxy-3-hexenoyl cyclohexenones: Diastereoselectivity in the intramolecular $[2+2]$ photocycloadditions of alkenes and cyclohexenones tethered by four atoms. Tetrahedron 1997, 53, 8963-8974.

7. Lásiková, A., Doháňošová, J., Hlavínová, L., Toffano, M., Vo-Thanh, G., Kožíšek, J., Gracza, T. Domino reaction: Pd(II)-catalyzed cyclization of unsaturated polyols and crosscoupling. Tetrahedron: Asymmetry 2012, 23, 818-827.

8. Elenkov, M. M., Hauer, B., Janssen, D. B. Enantioselective Ring Opening of Epoxides with Cyanide Catalysed by Halohydrin Dehalogenases: A New Approach to Non-Racemic $\beta$ Hydroxy Nitriles. Adv. Synth. Catal. 2006, 348, 579-585.

9. Moss, T. A.; Alonso, B.; Fenwick, D. R.; Dixon, D. J. Catalytic Enantio- and Diastereoselective Alkylations with Cyclic Sulfamidates. Angew. Chem. Int. Ed. 2010, 49, 568571.

10. Cooper, M. A., Ward, A. D. Cyclizations using Selenium Chemistry for Substituted 3Hydroxypiperidines and 3-Hydroxypyrrolidines. Aust. J. Chem. 2011, 64, 1327-1338.

11. Paddon-Jones, G. C., McErlean, C. S. P., Hayes, P., Moore, C. J., Konig, W. A., Kitching, W. Synthesis and Stereochemistry of Some Bicyclic $\gamma$-Lactones from Parasitic Wasps (Hymenoptera: Braconidae). Utility of Hydrolytic Kinetic Resolution of Epoxides and Palladium(II)-Catalyzed Hydroxycyclization-Carbonylation-Lactonization of Ene-diols. $J$. Org. Chem. 2001, 66, 7487-7495.

12. Tamaru, Y.; Kobayashi, T.; Kawamura, S.-I.; Ochiai, H.; Hojo, M.; Yoshida, Z.-I. Palladium catalyzed oxycarbonylation of 4-penten-1,3-diols: efficient stereoselective synthesis of cis 3-hydroxytetrahydrofuran 2-acetic acid lactones. Tetrahedron Lett. 1985, 26, 3207-3210 13. Tamaru, Y.; Hojo, M.; Kawamura, S.; Sawada, S.; Yoshida, Z. Stereoselective intramolecular iodoetherification of 4-pentene-1,3-diols: Synthesis of cis-2-(iodomethyl)-3hydroxytetrahydrofurans J. Org. Chem. 1987, 52, 4062-4072.

14. Leijondahl K., Borén L., Braun R., Bäckvall J.-E. Enantiopure 1,5-Diols from Dynamic Kinetic Asymmetric Transformation. Useful Synthetic Intermediates for the Preparation of Chiral Heterocycles. Org. Lett. 2008, 10, 2027-2030. 


\section{Copies of ${ }^{1} \mathrm{H}$ and ${ }^{13} \mathrm{C}$ NMR spectra}

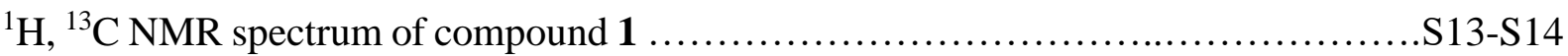

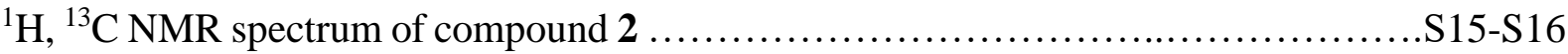

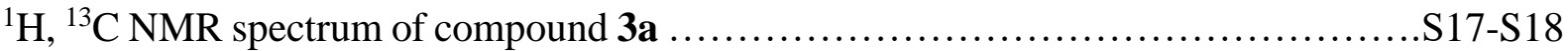

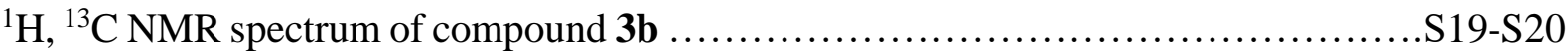

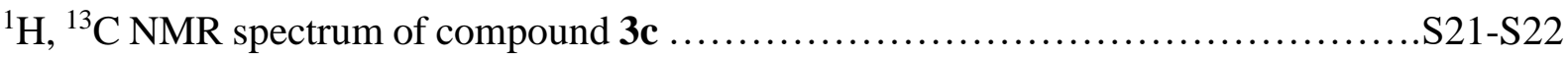

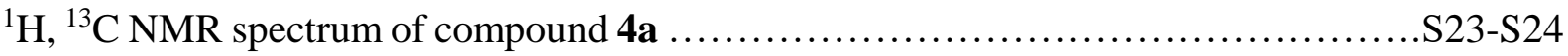

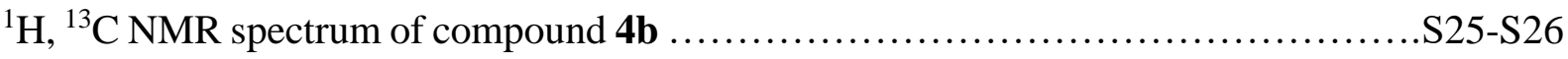

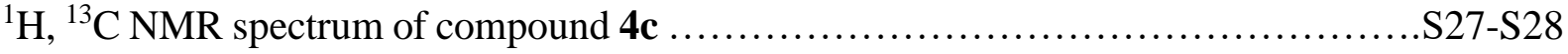

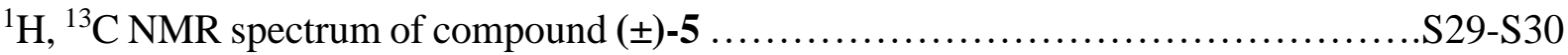

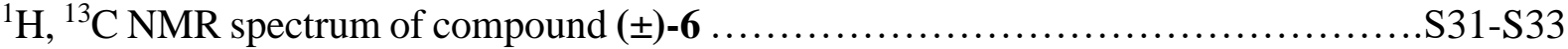

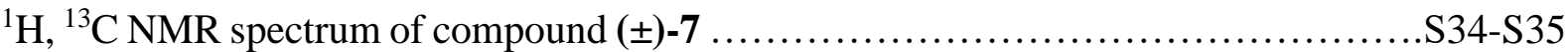

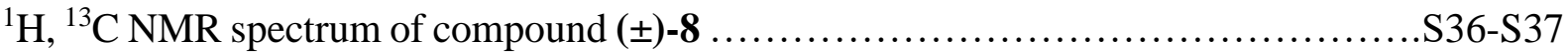

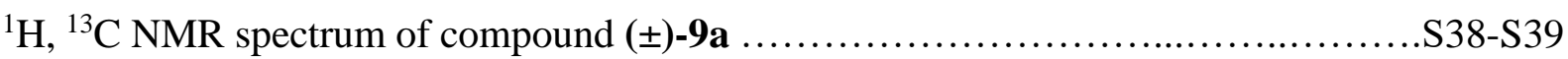

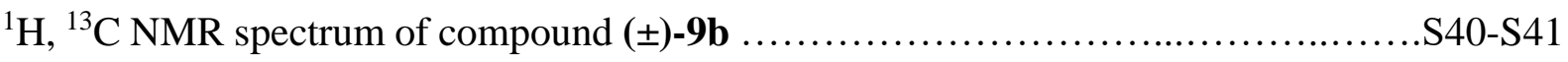

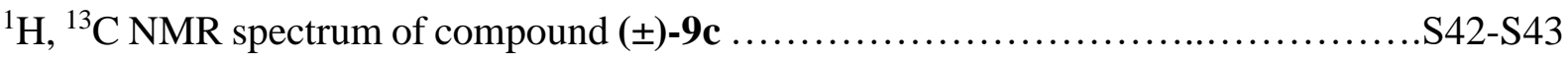

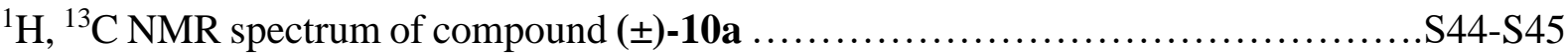

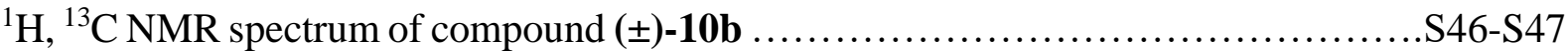

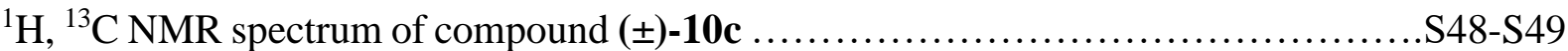

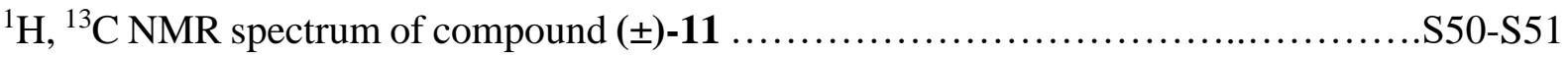

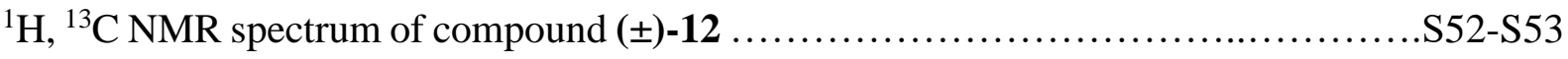

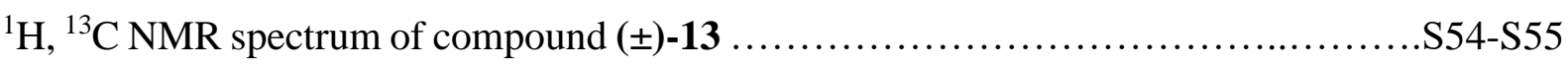

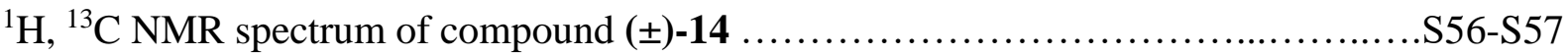

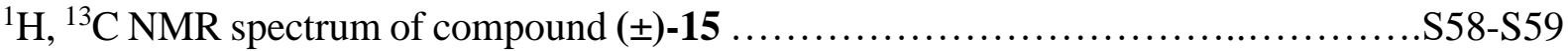

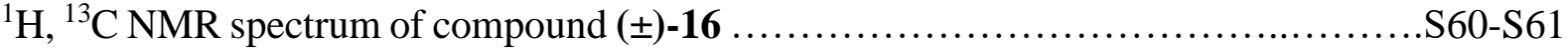

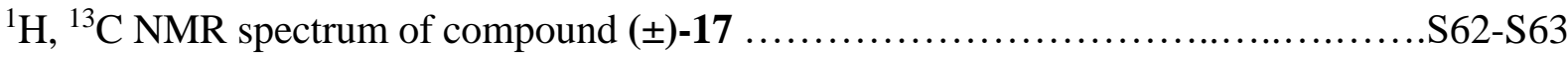

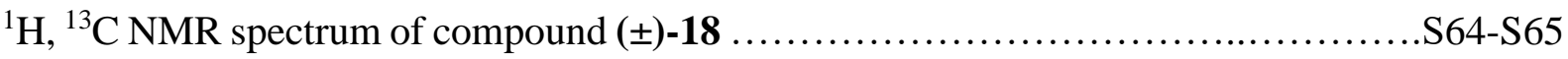

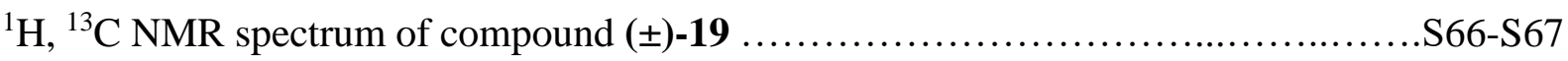

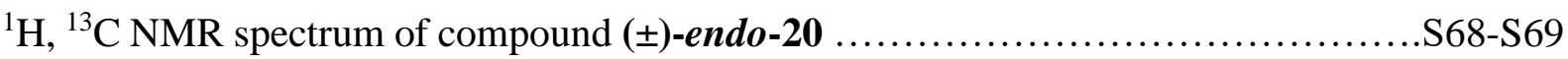

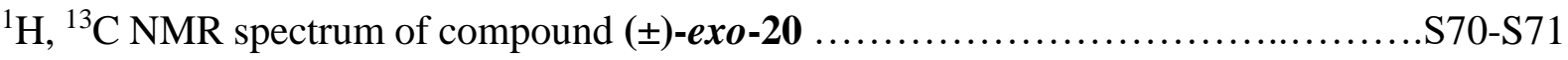

${ }^{1} \mathrm{H},{ }^{13} \mathrm{C}$ NMR spectrum of compound D-ido/D-galacto-22 ........................... 72 .S73

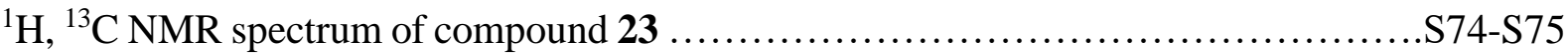




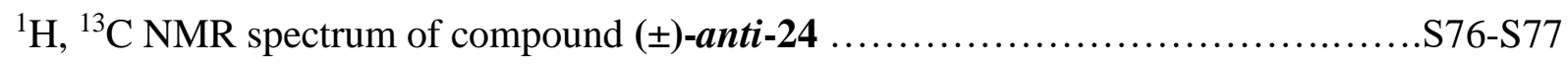

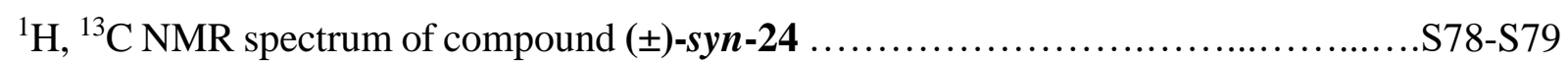

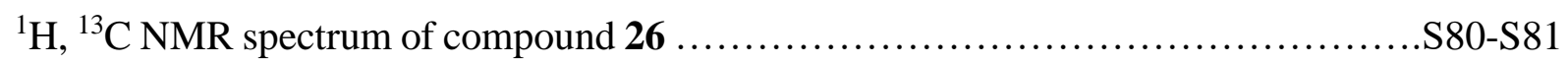

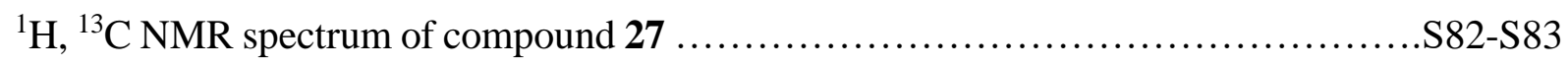

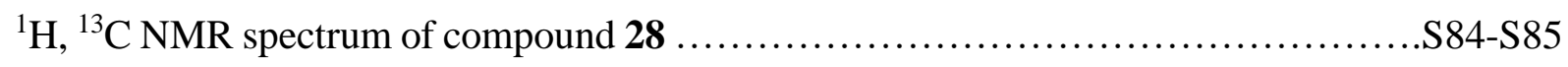

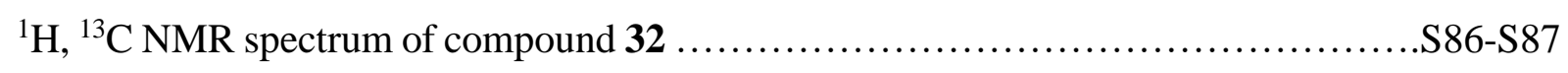

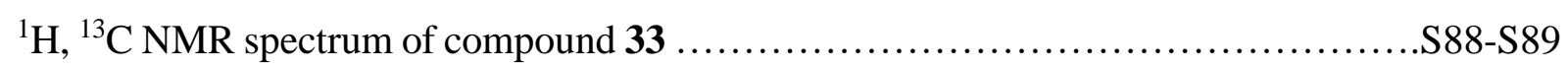

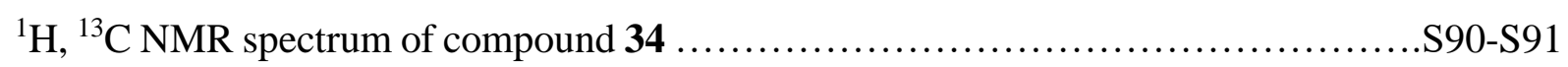

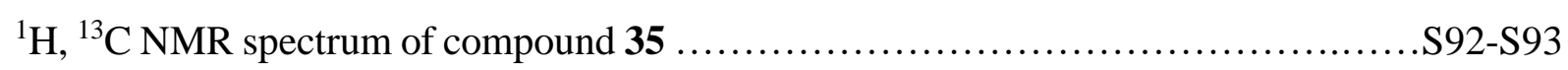

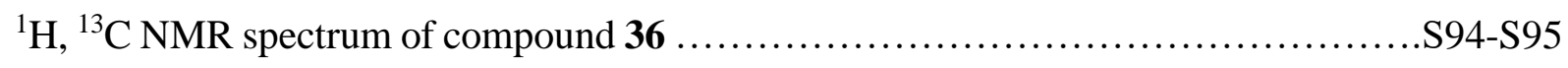



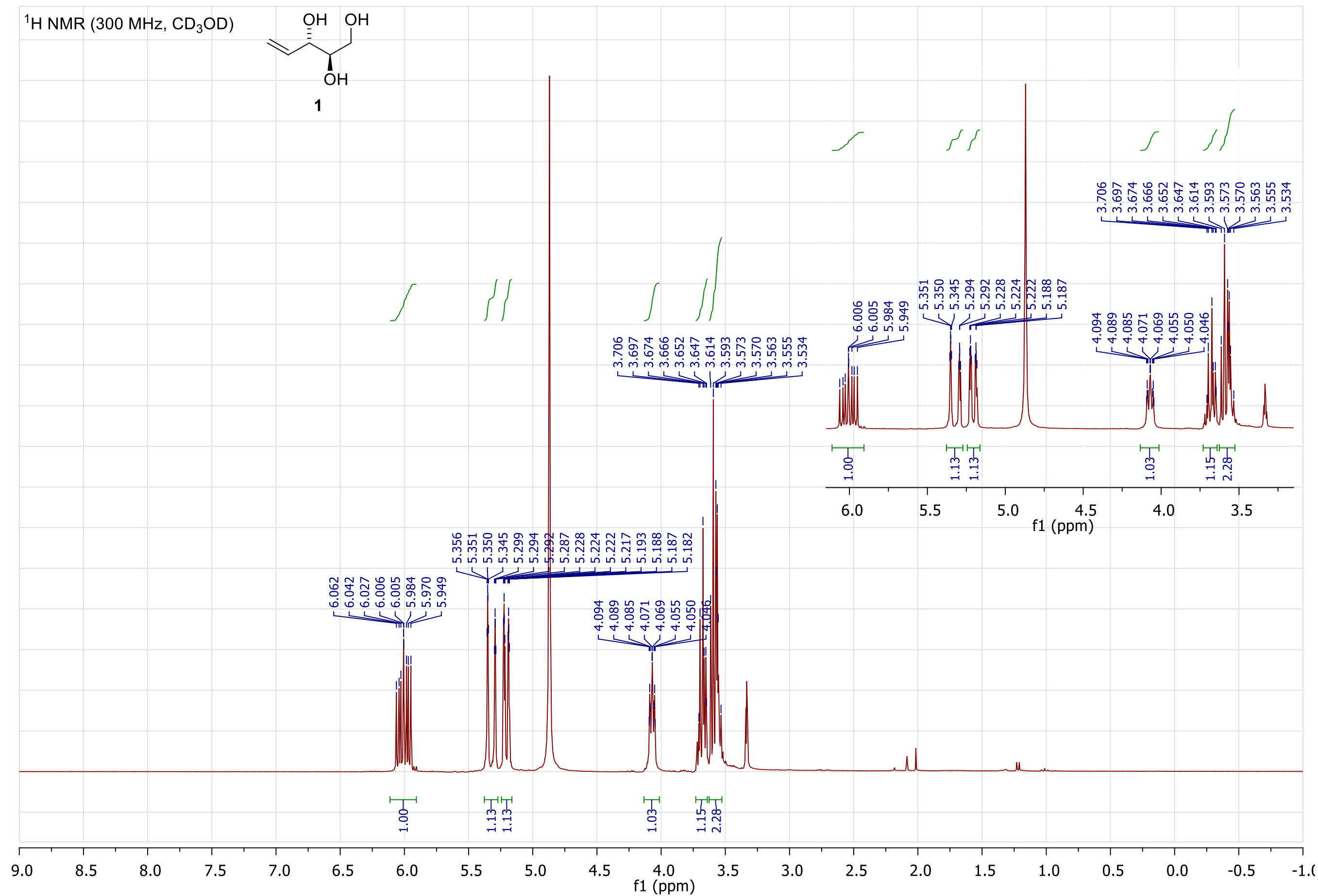


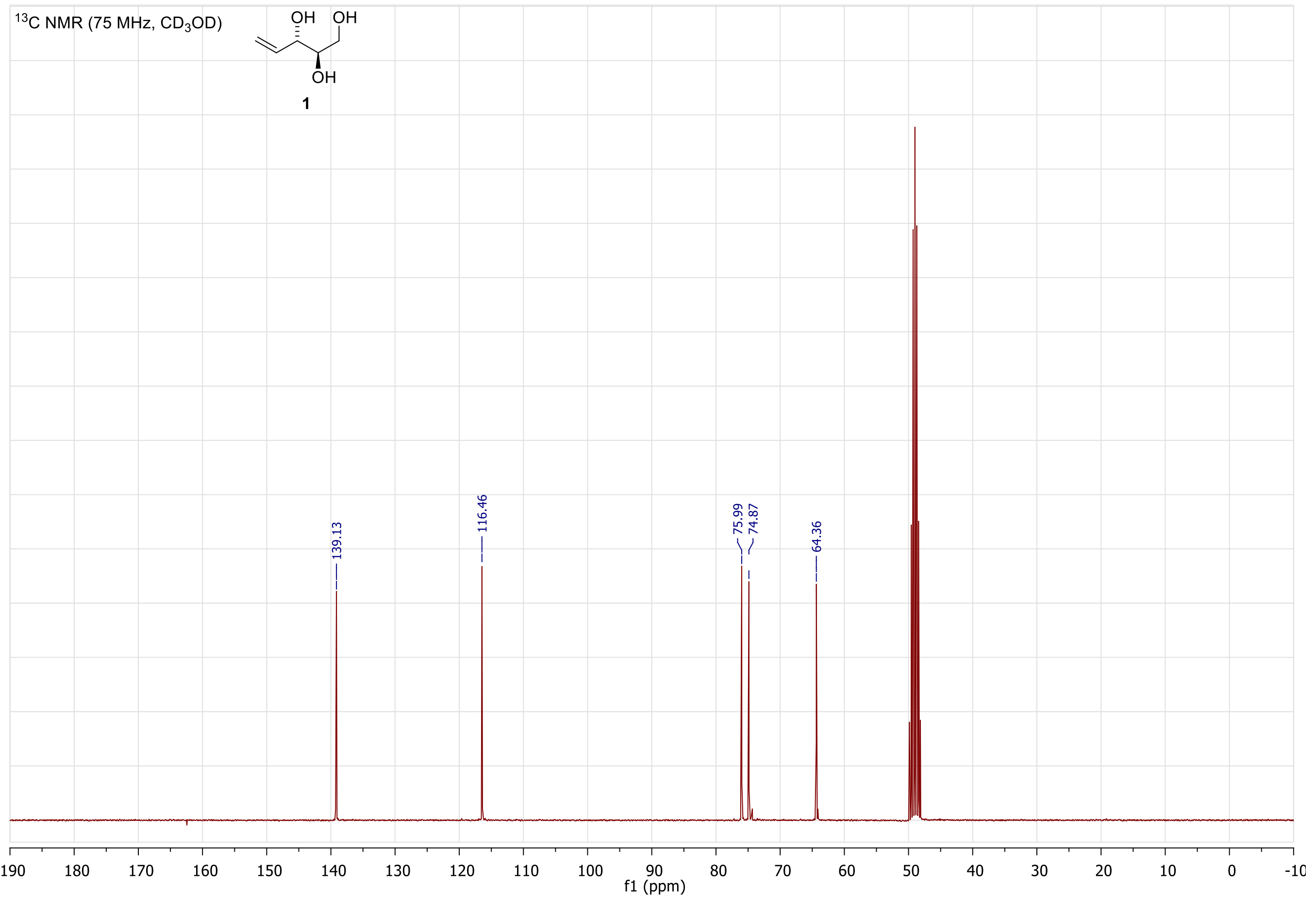

S14 


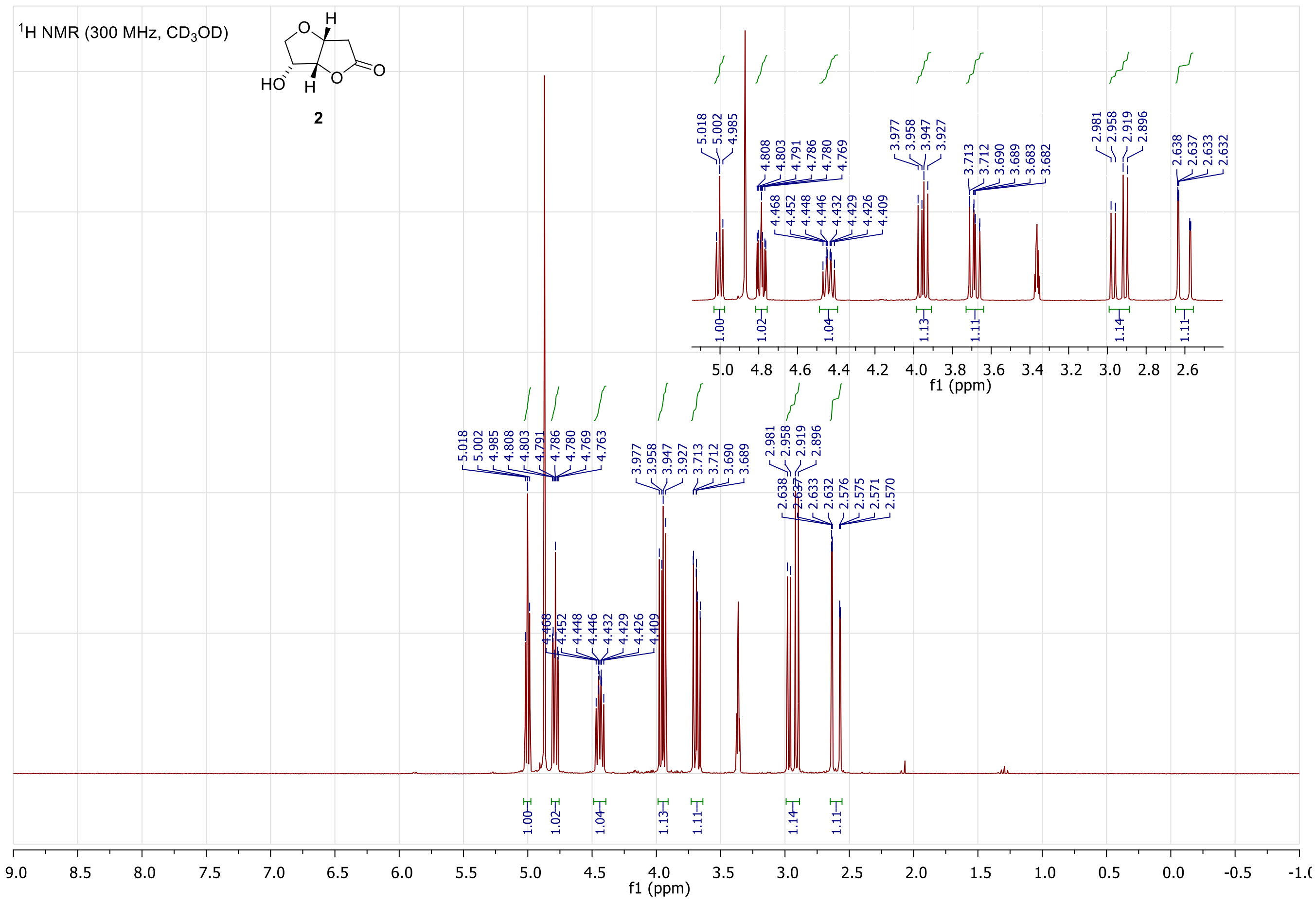




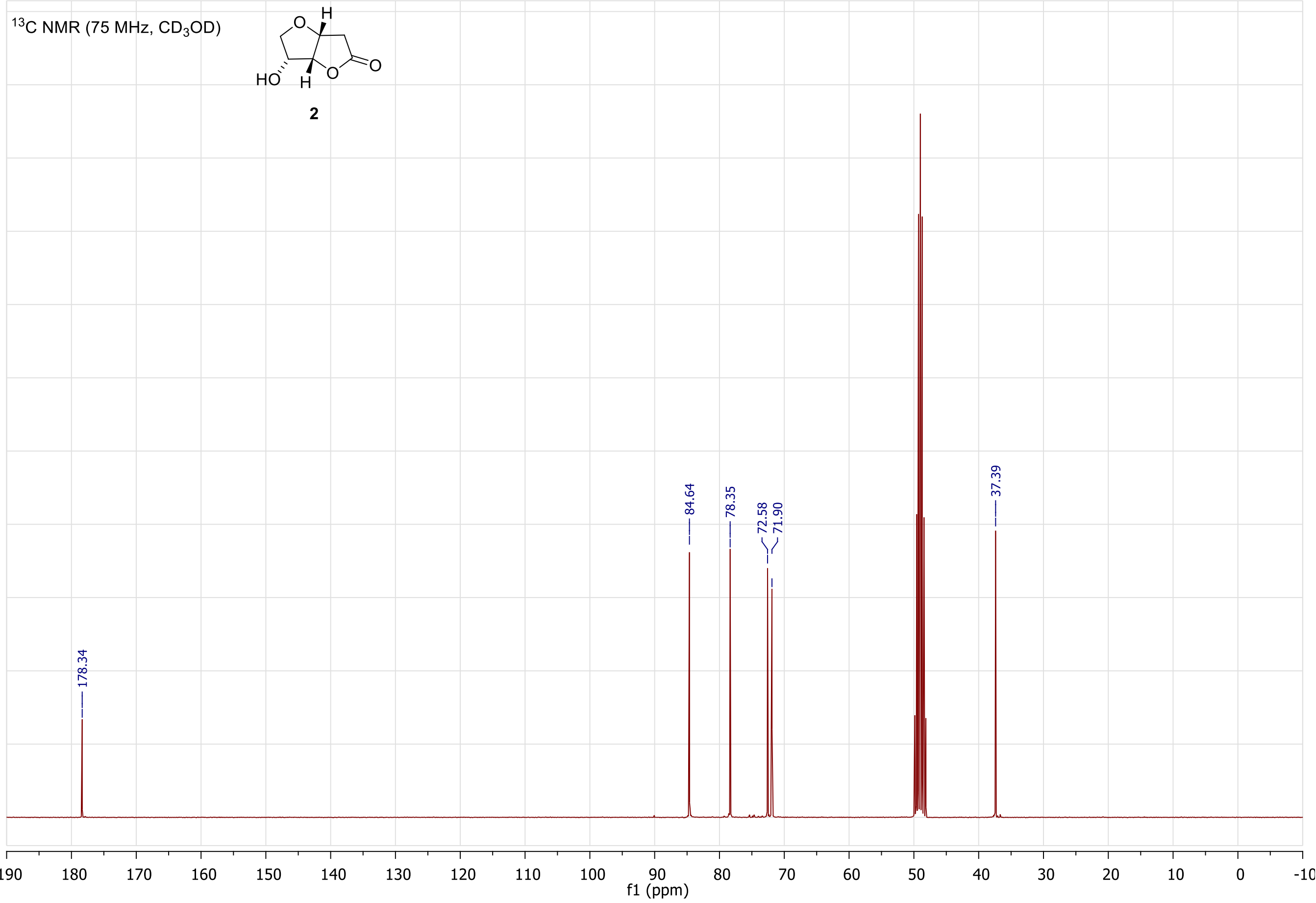




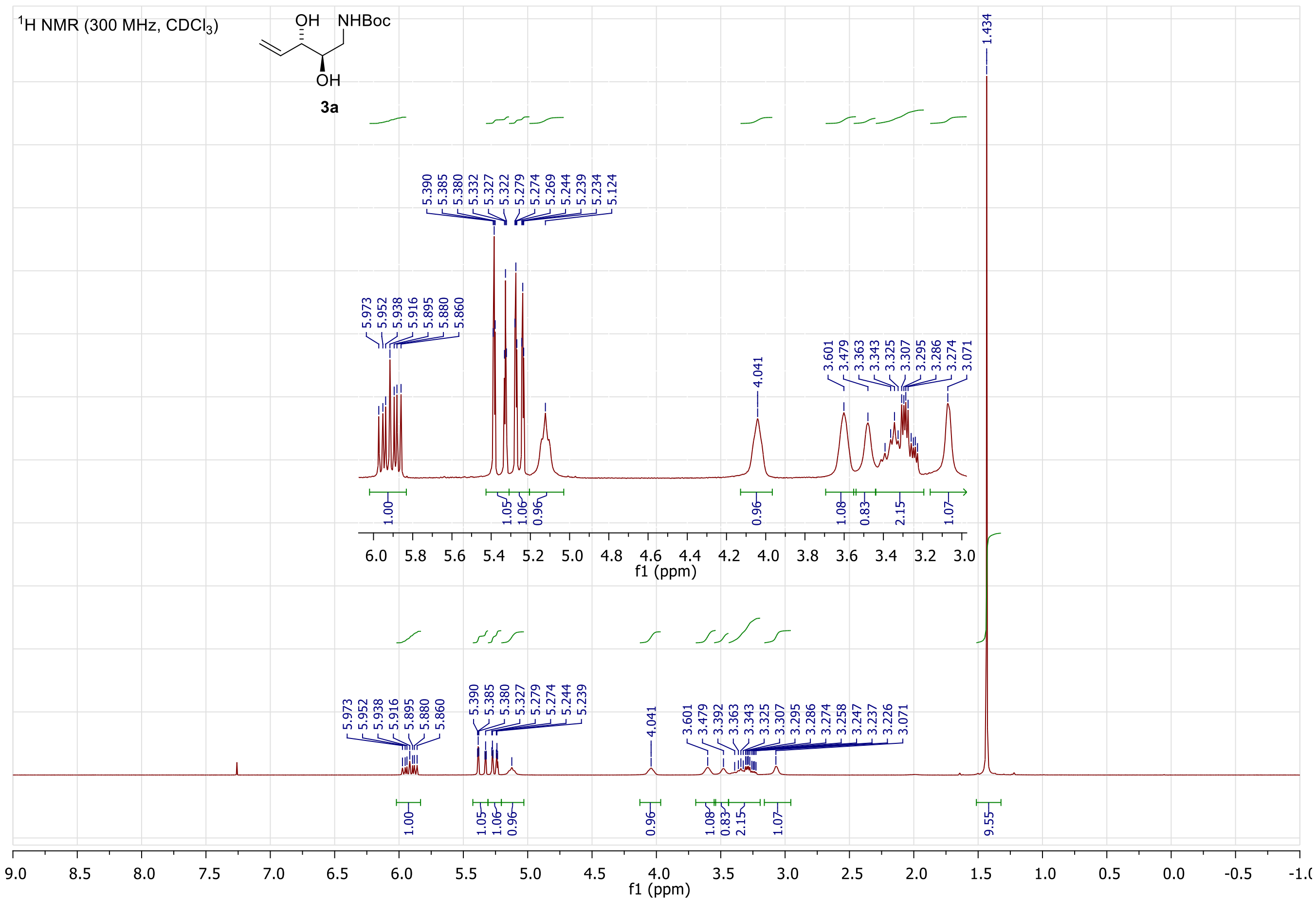




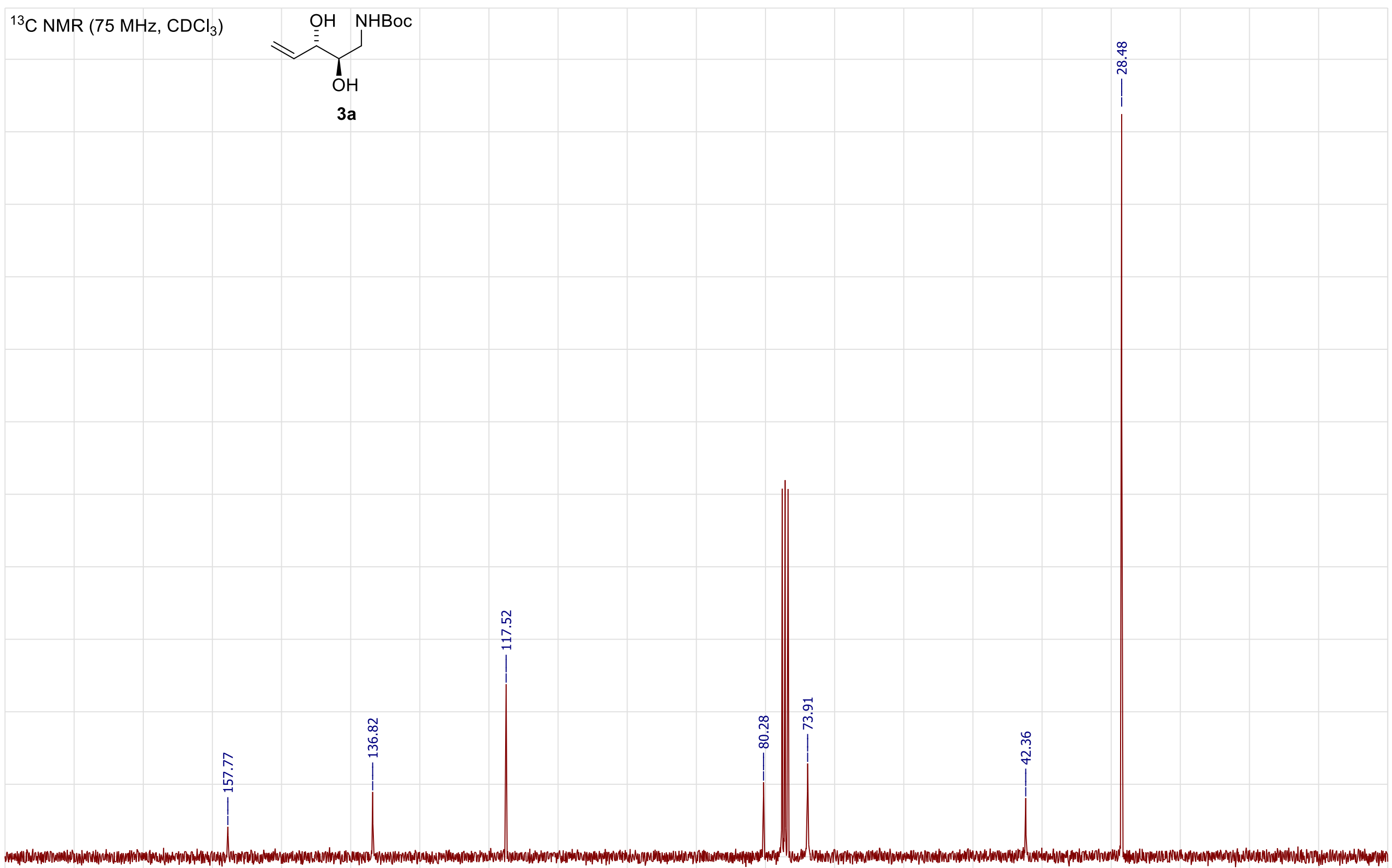




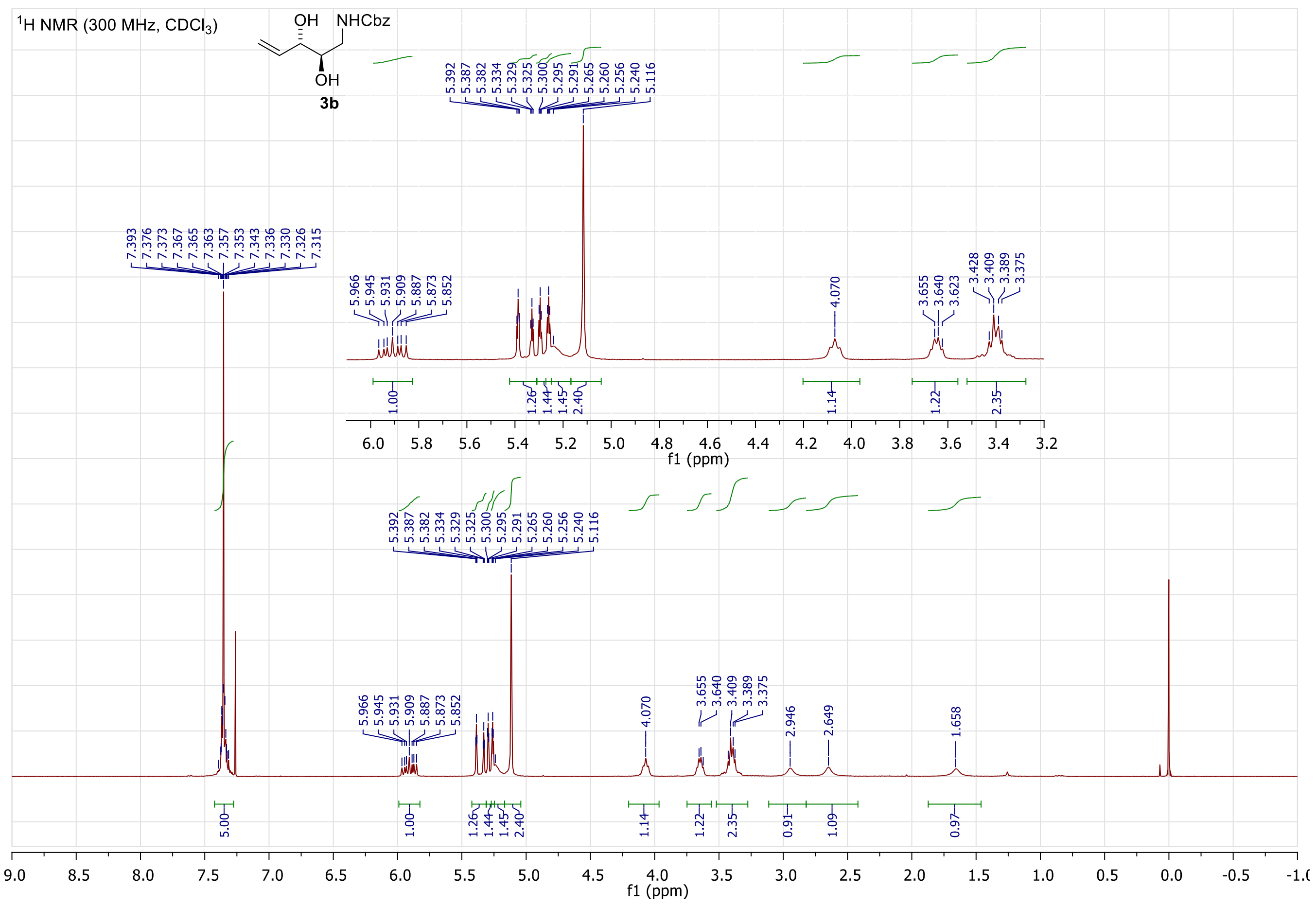




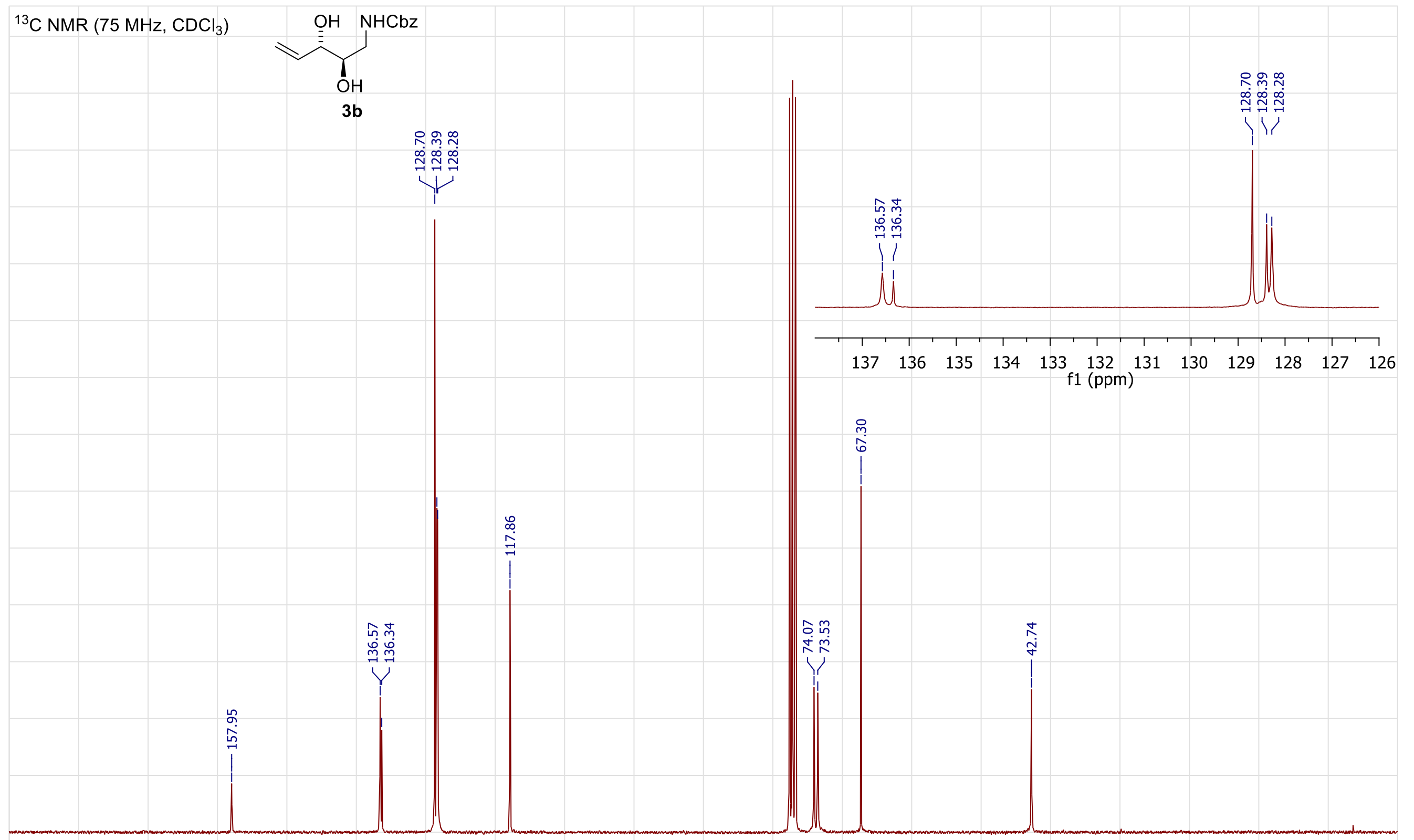

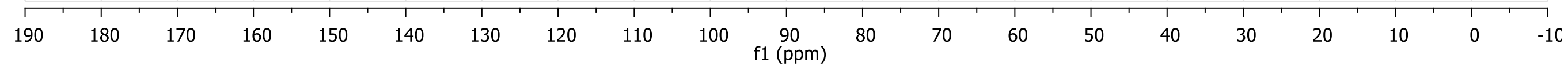




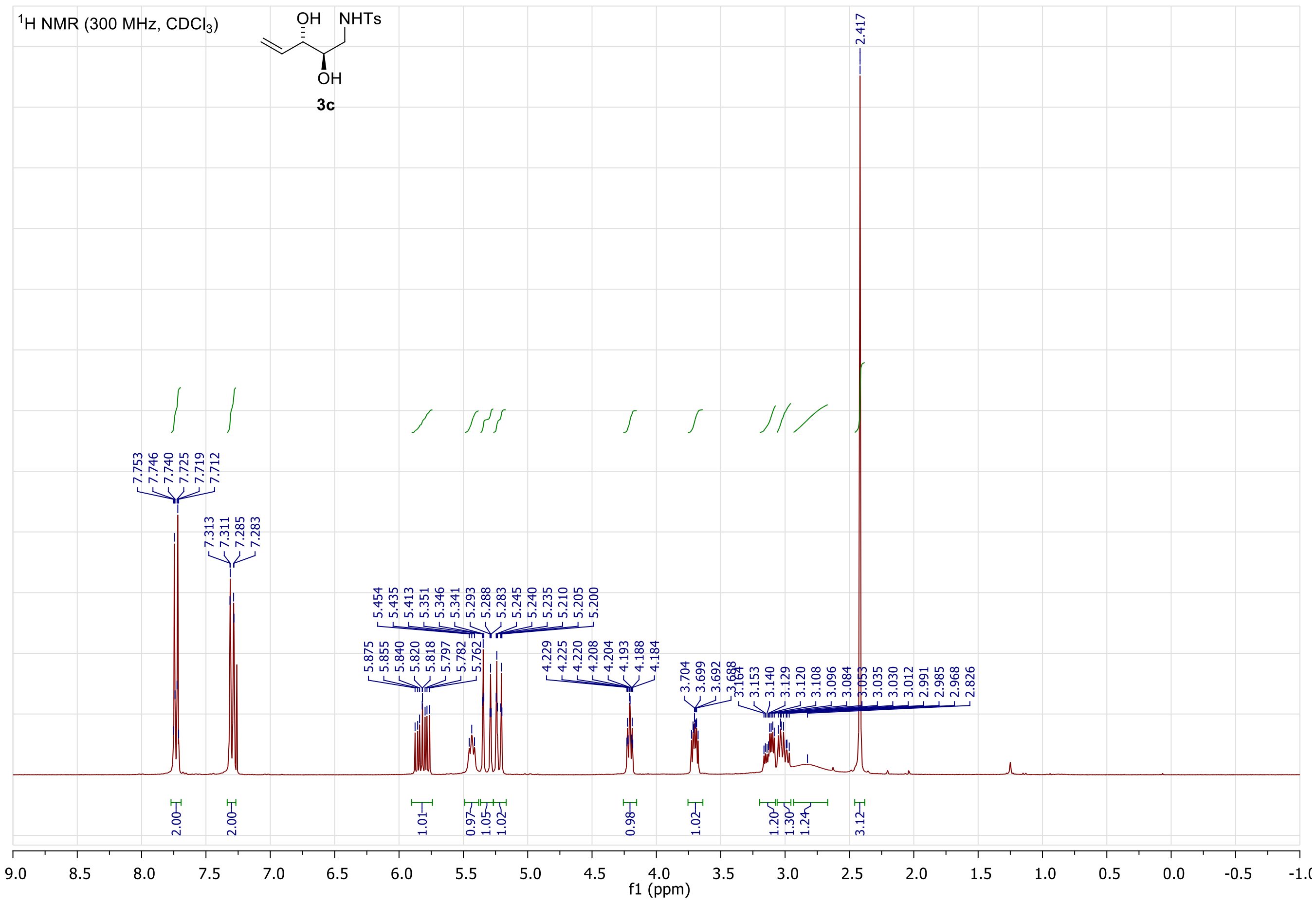




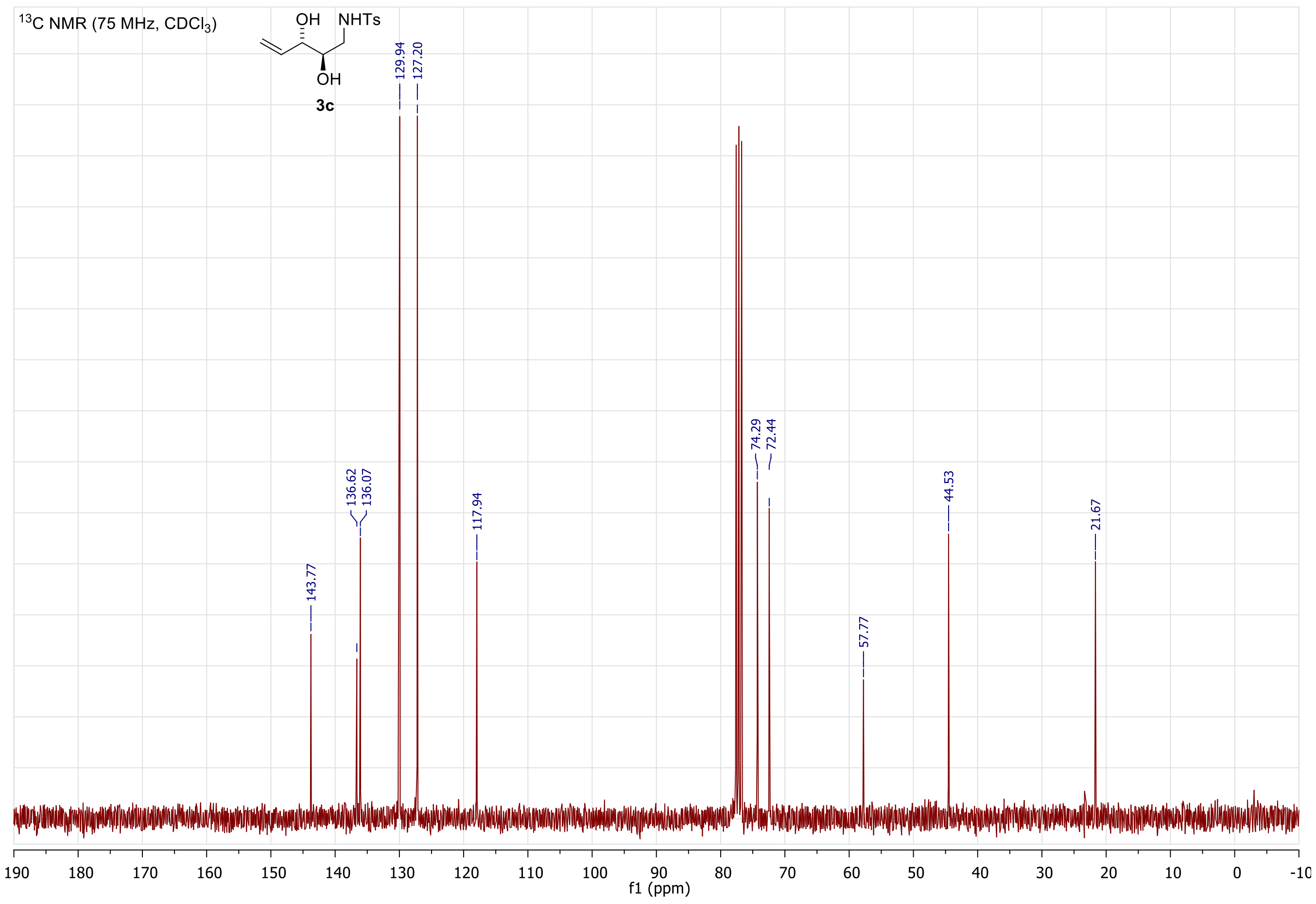




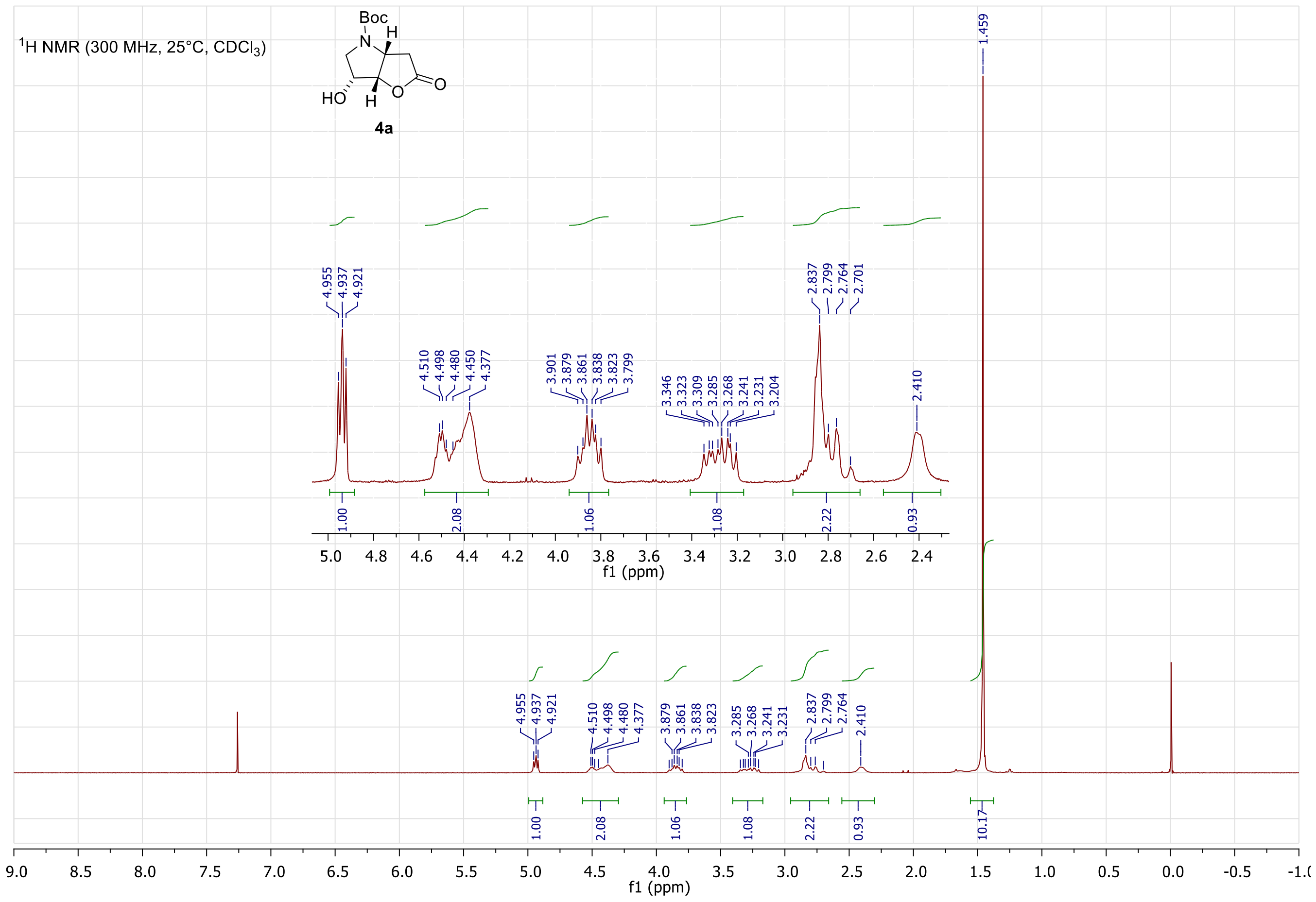




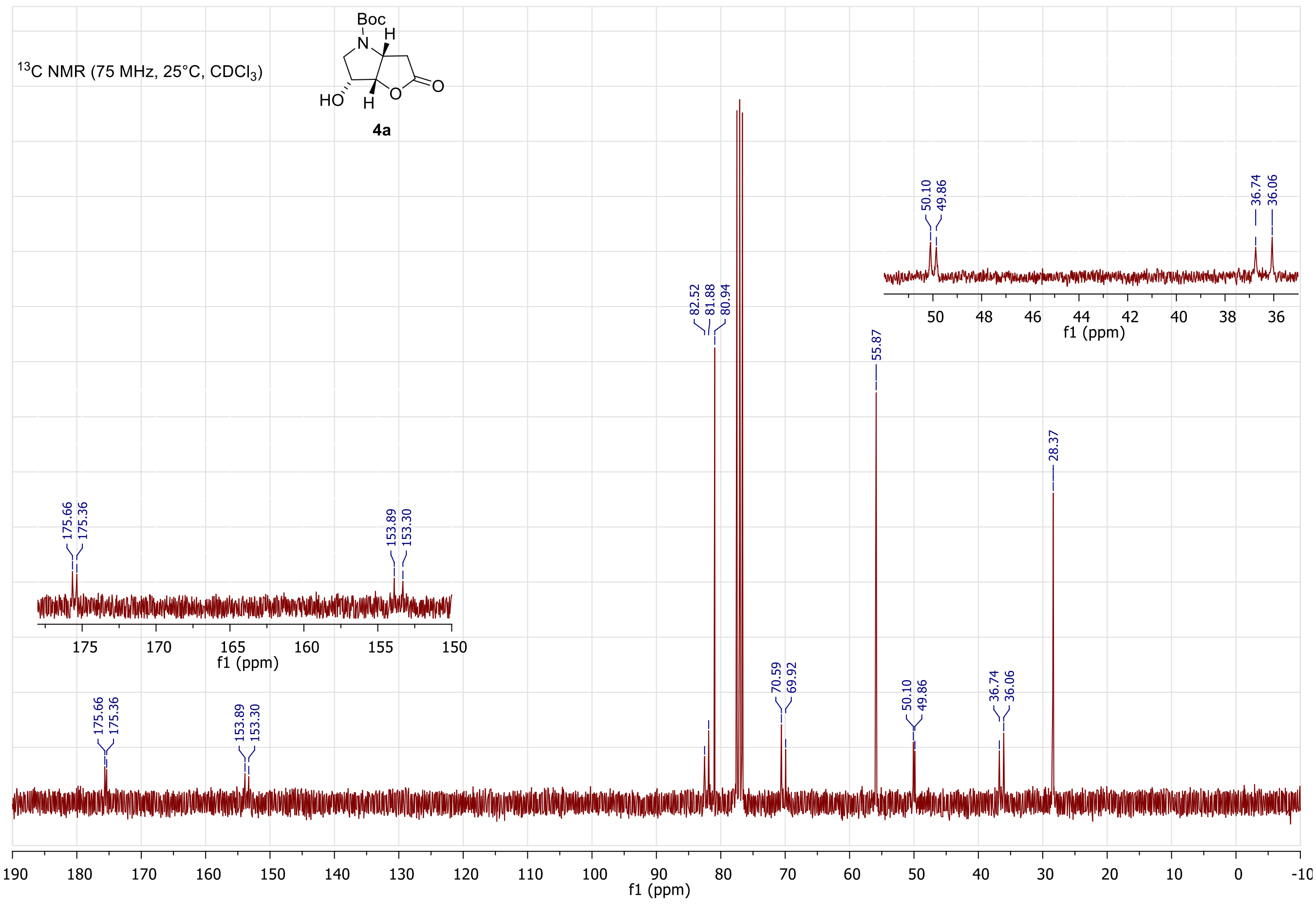




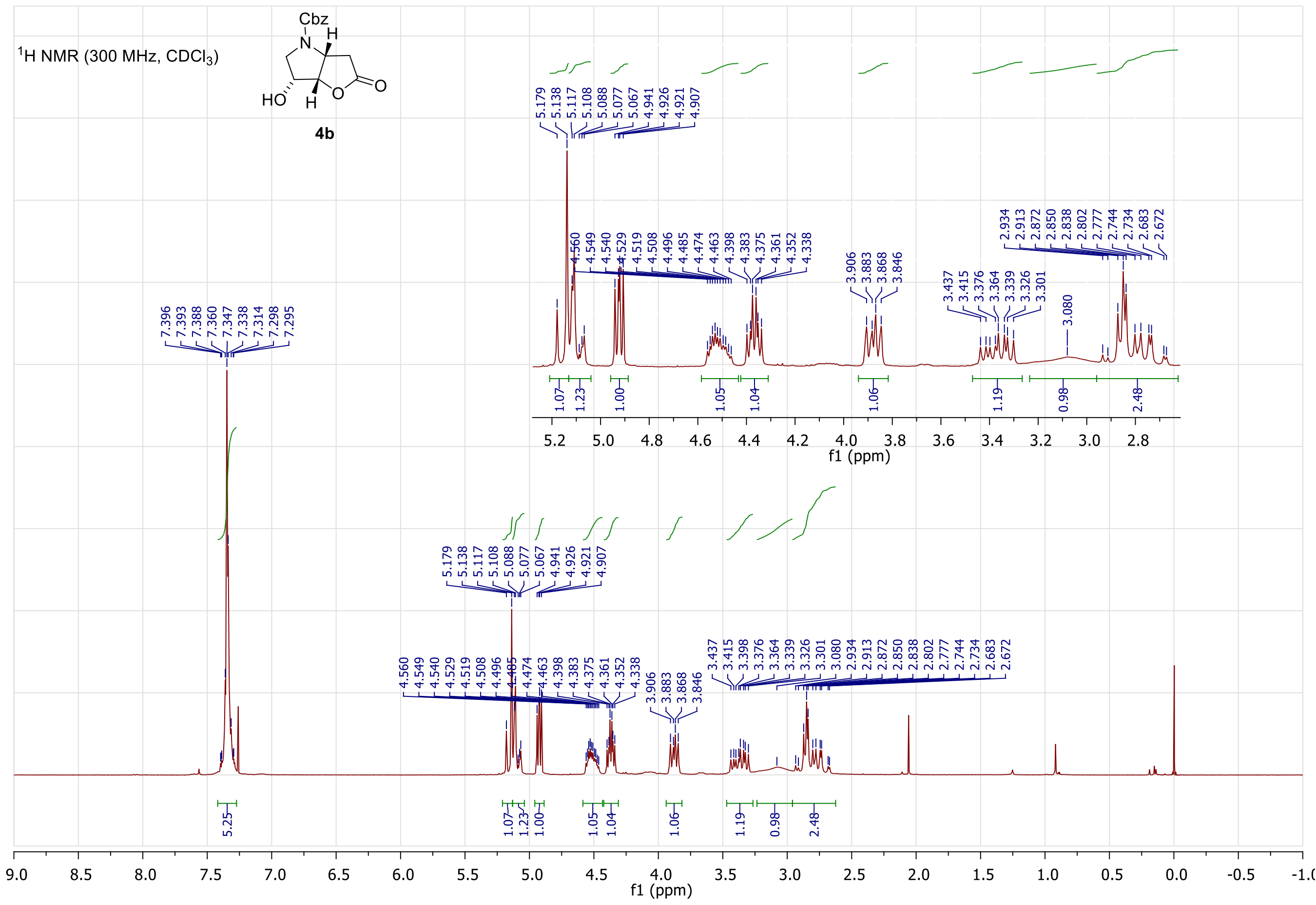




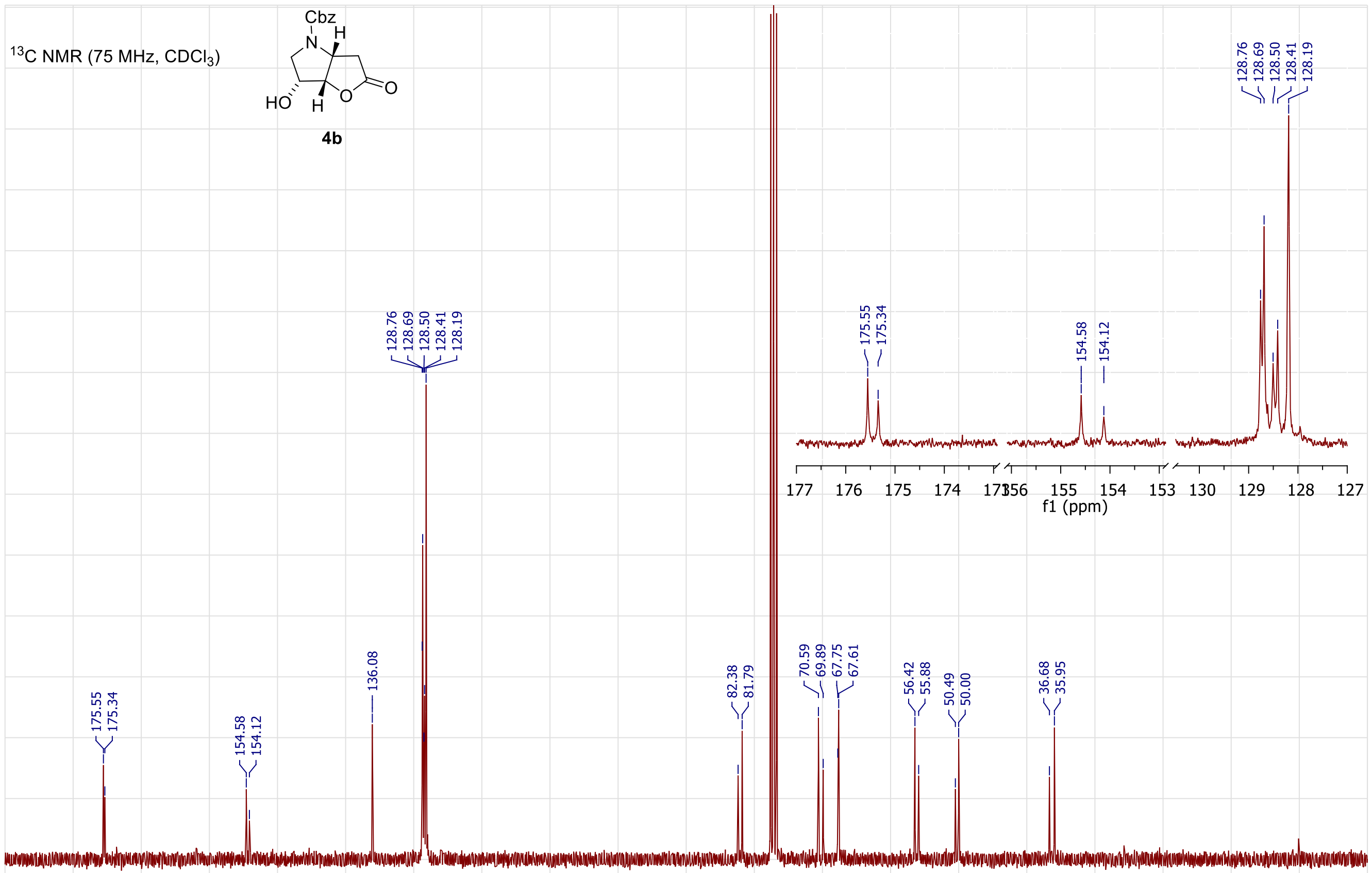




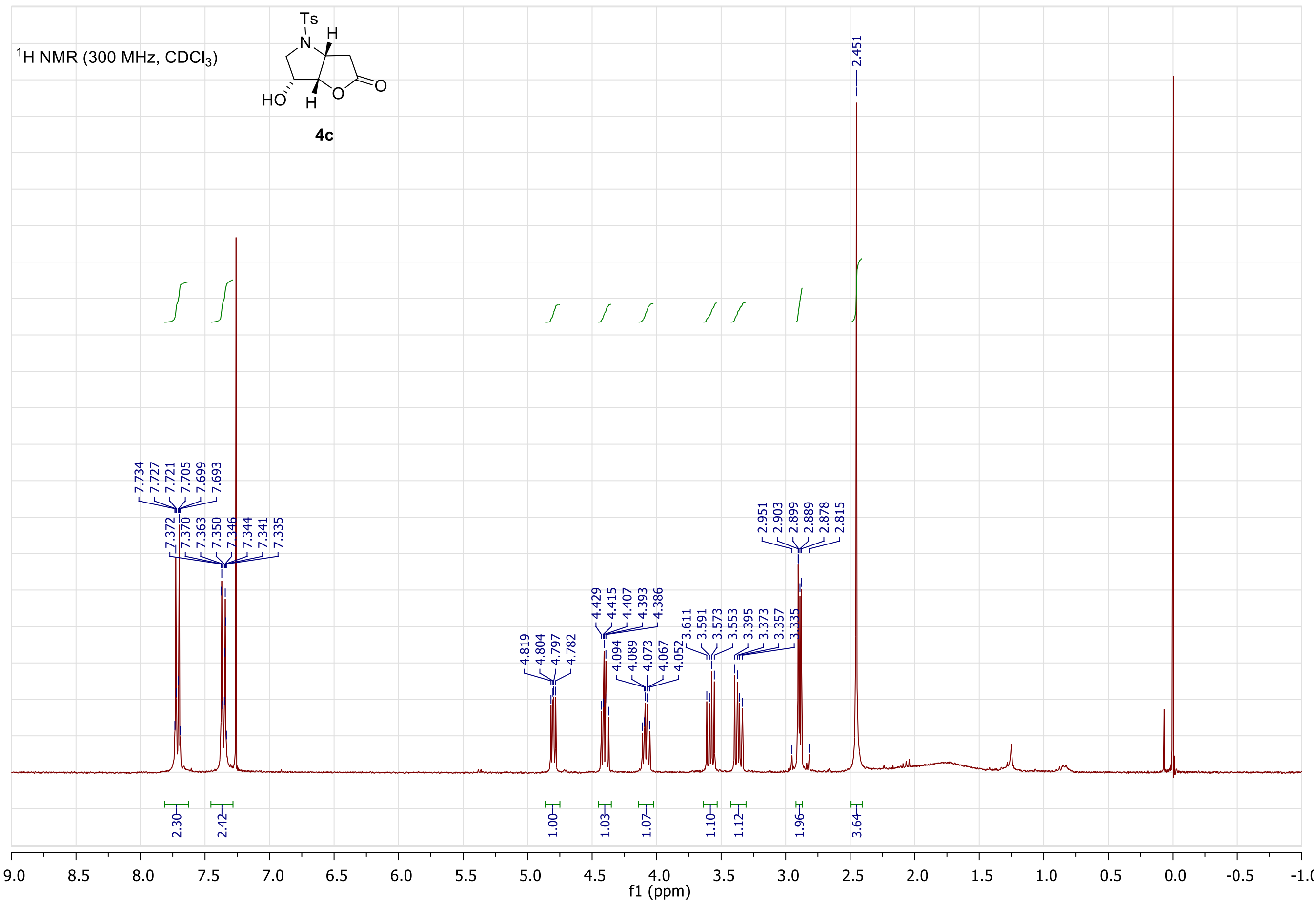




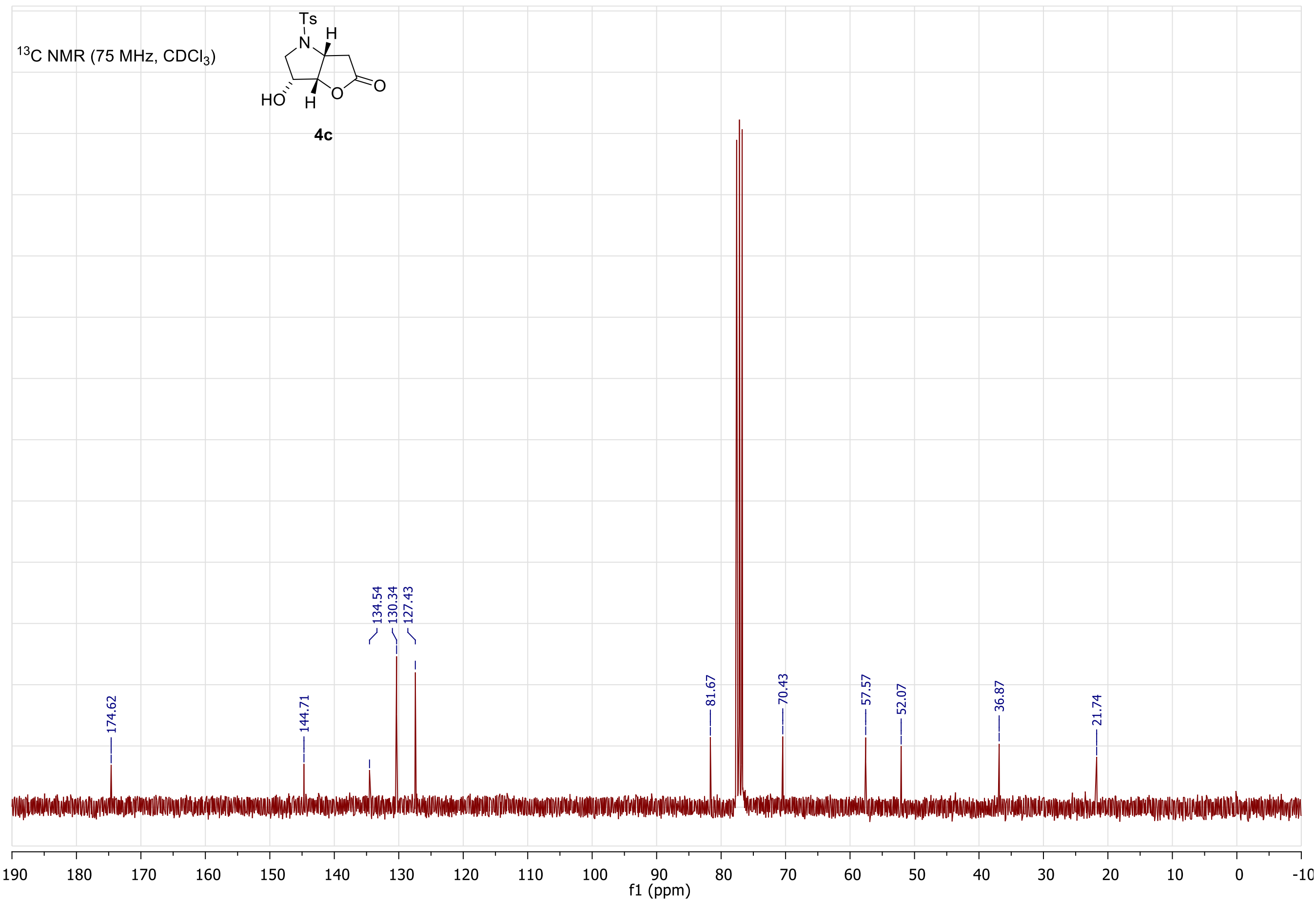




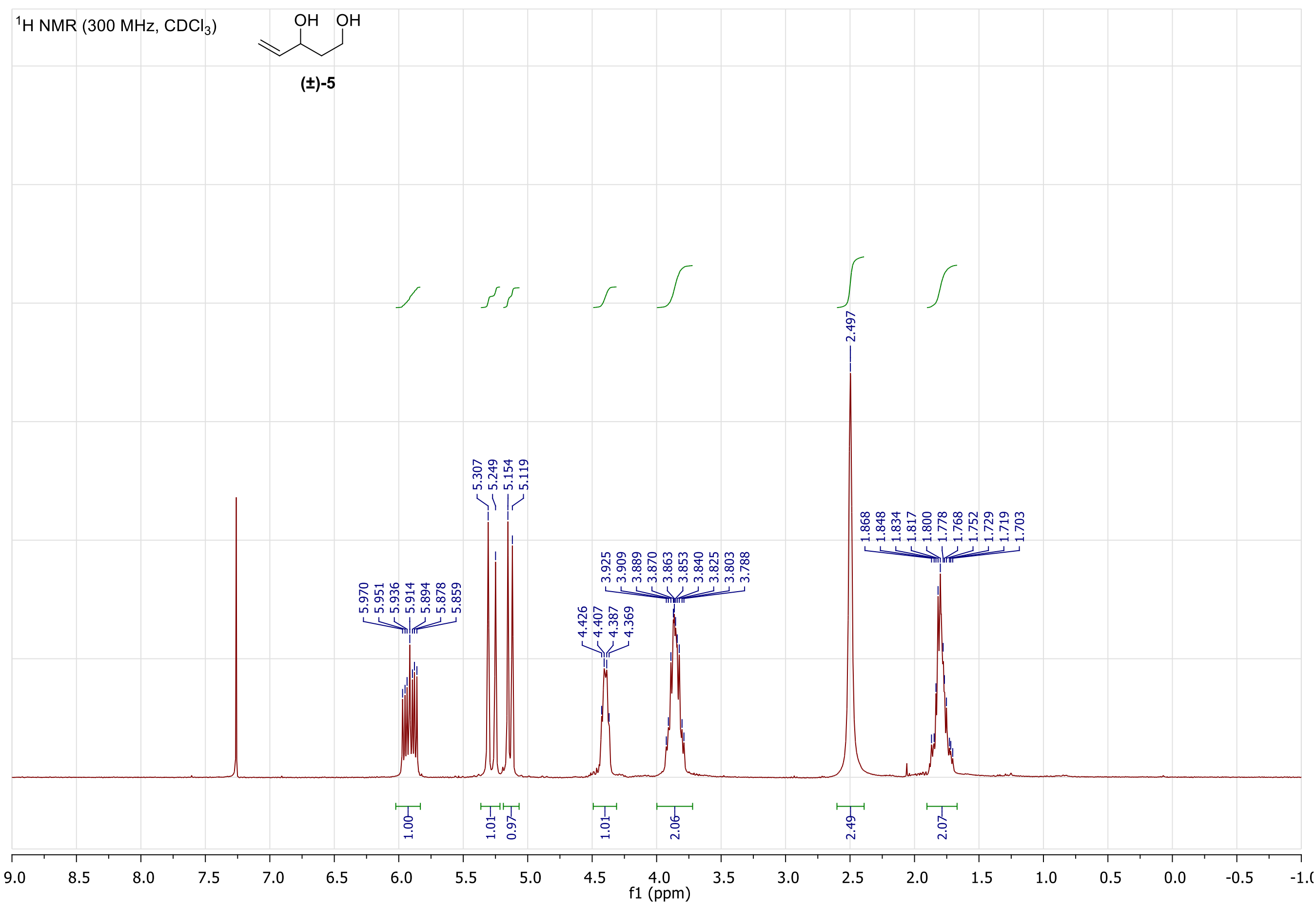




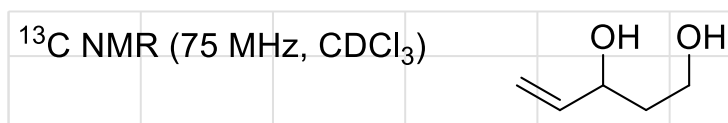

$( \pm)-5$

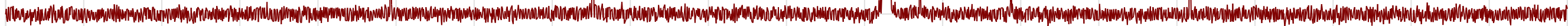

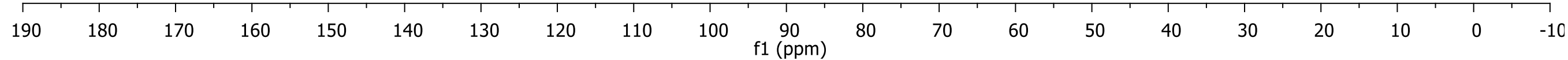




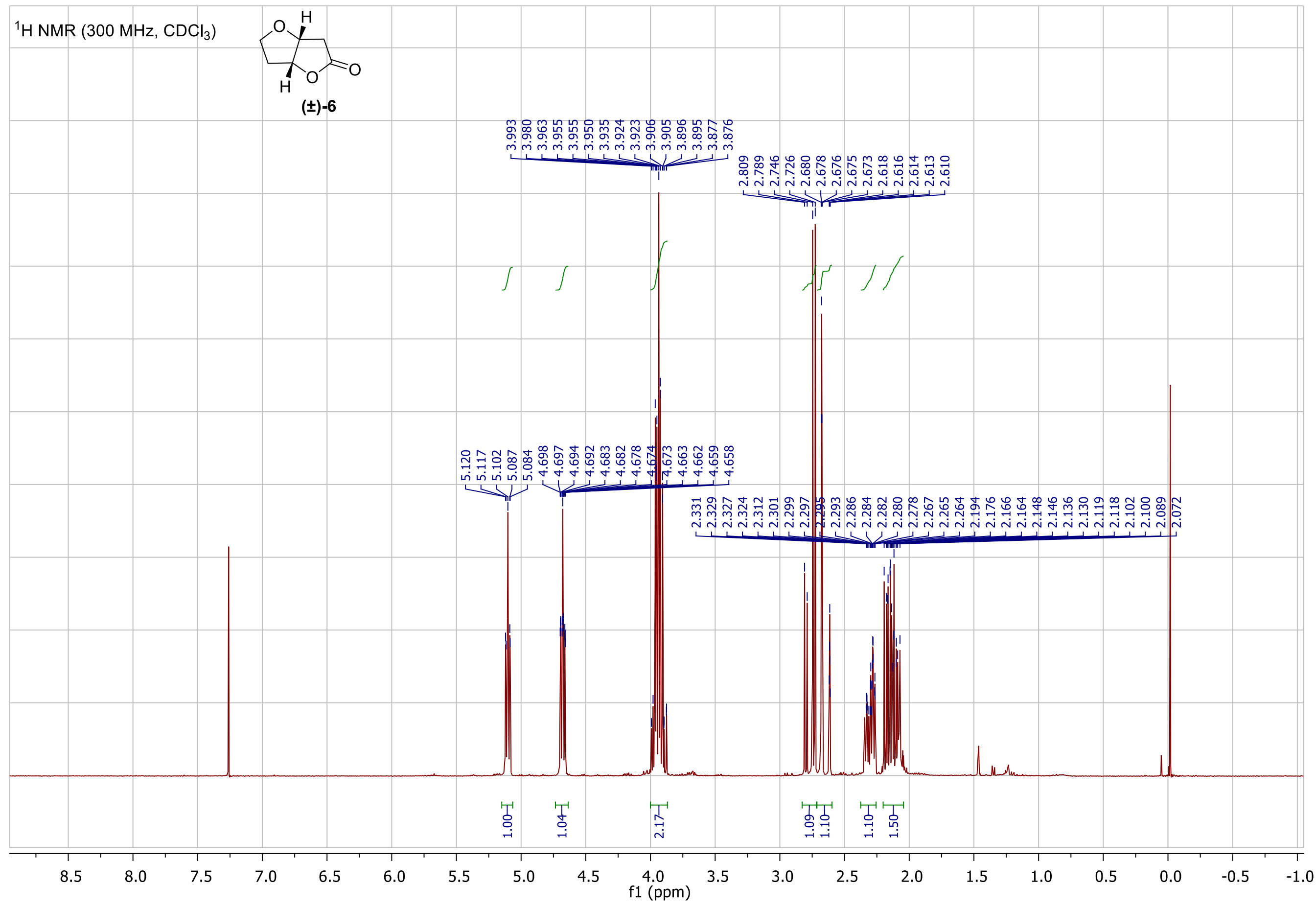




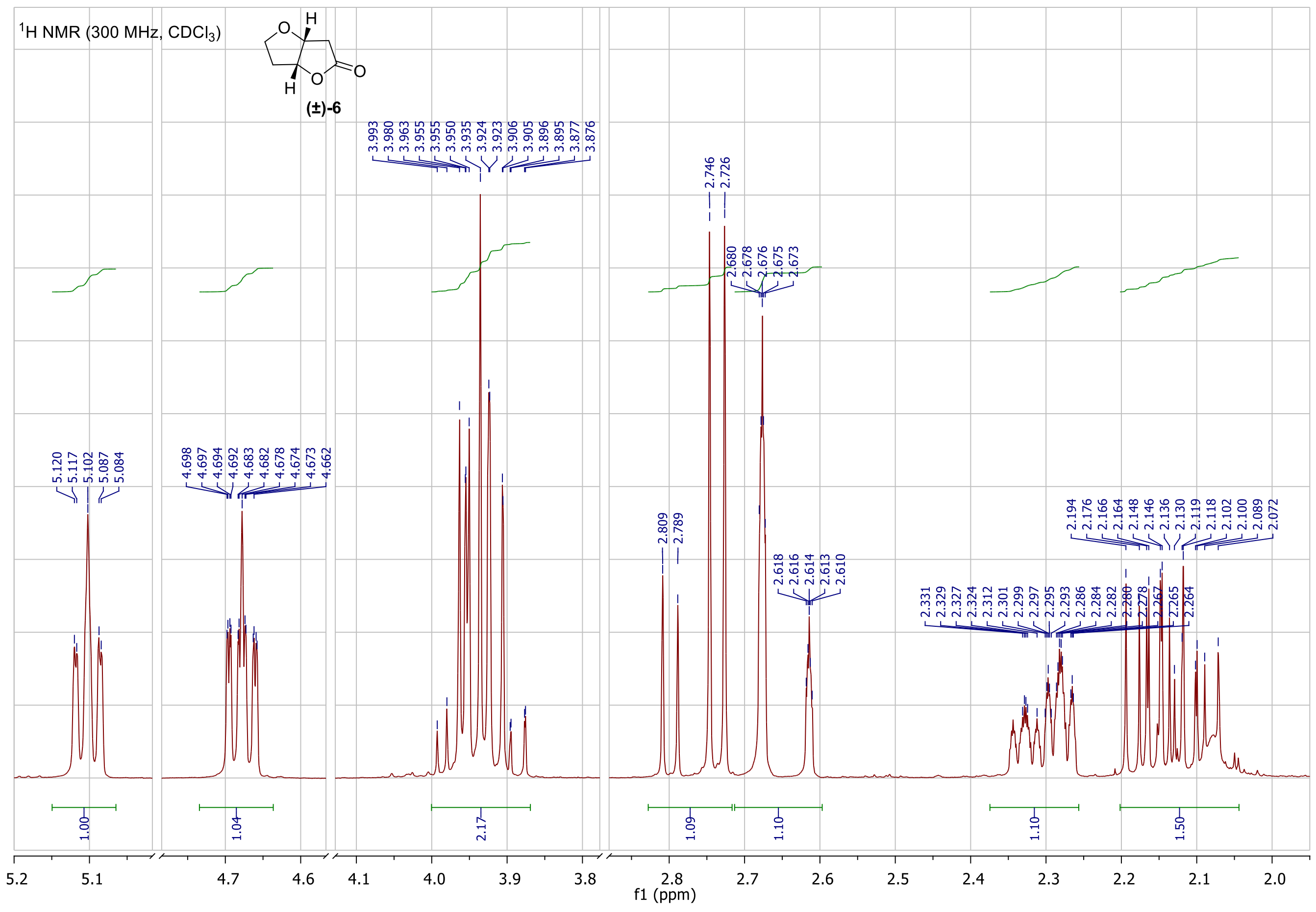




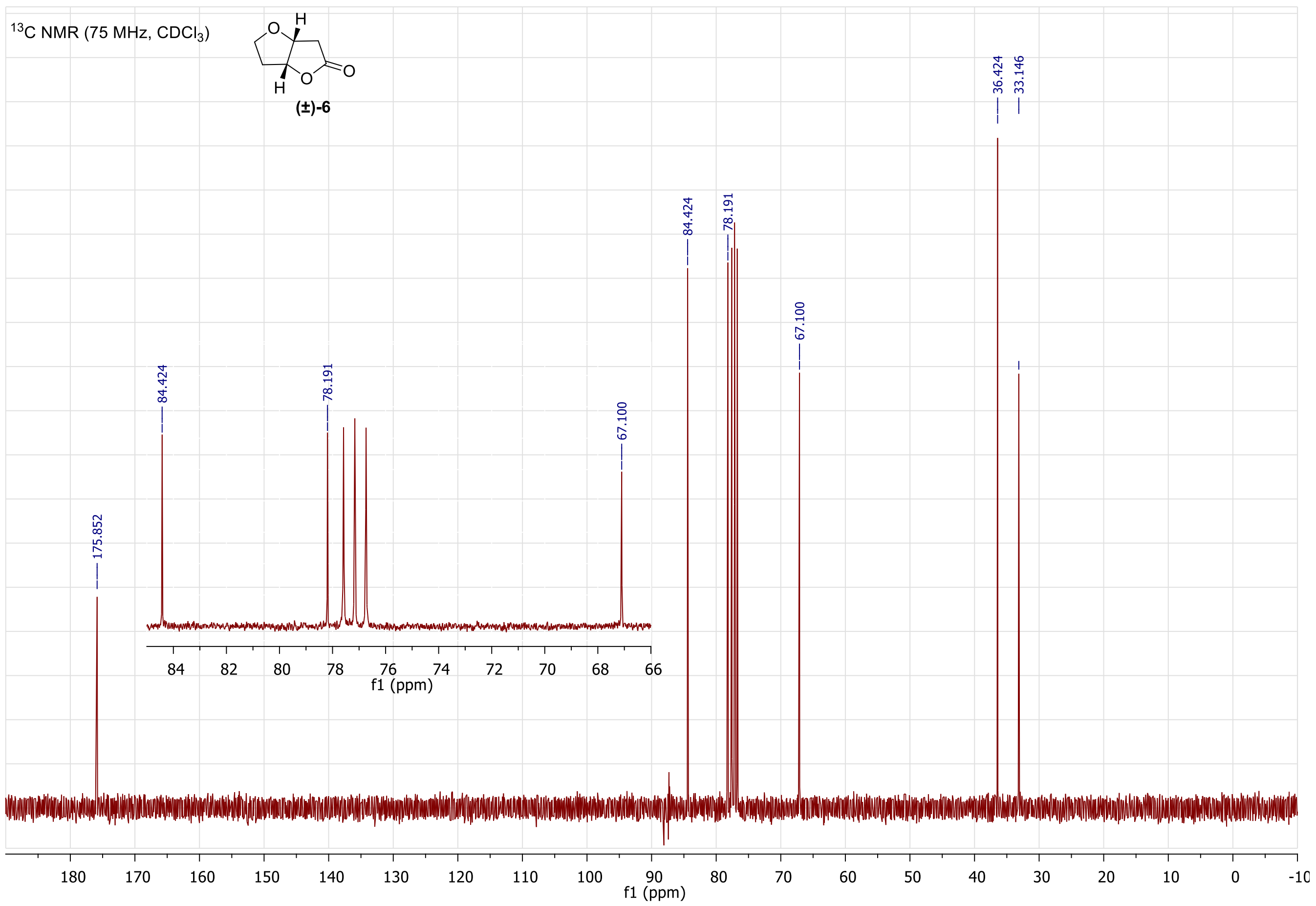

S33 


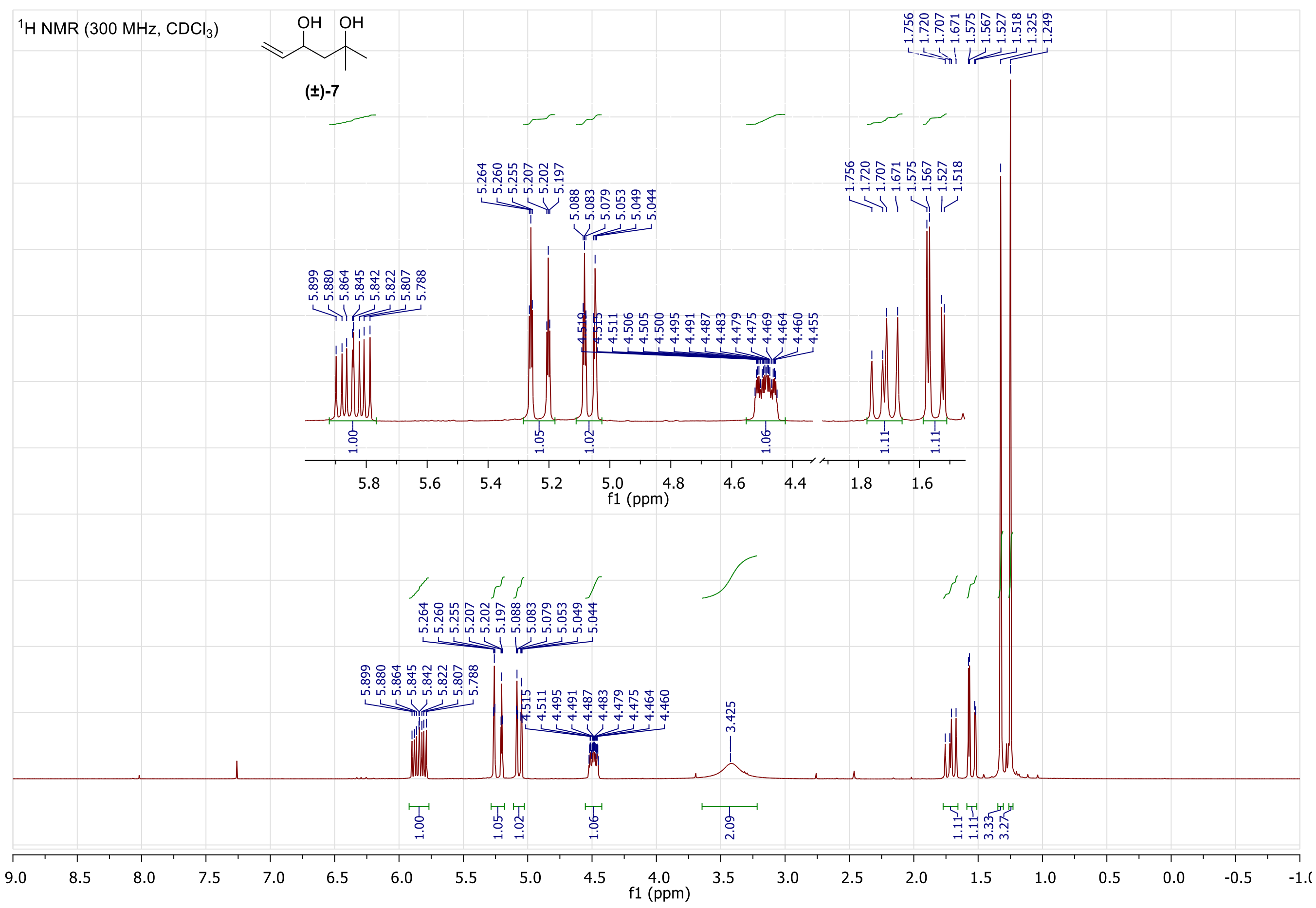




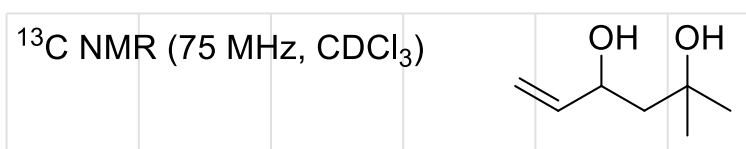

(士)-7

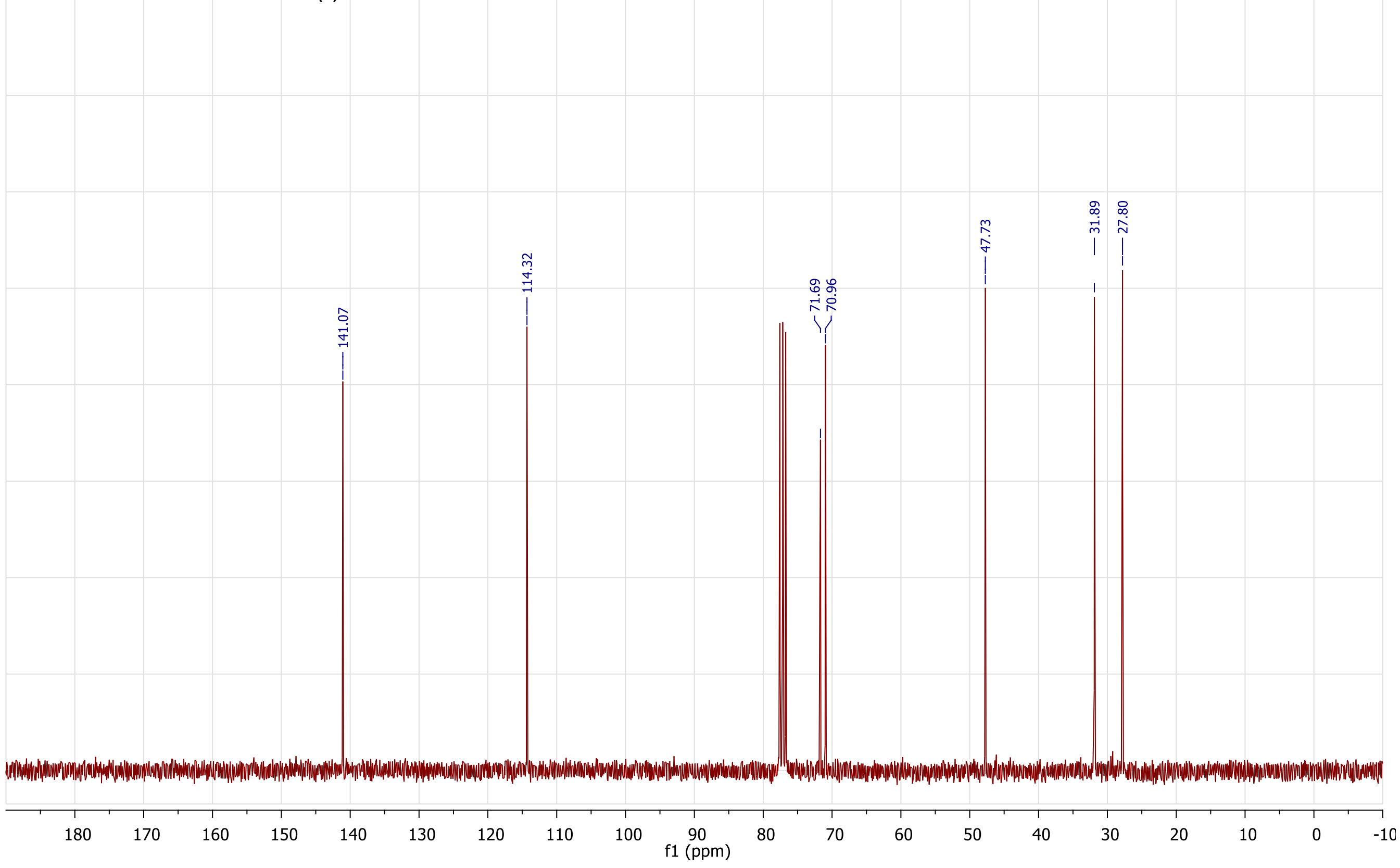




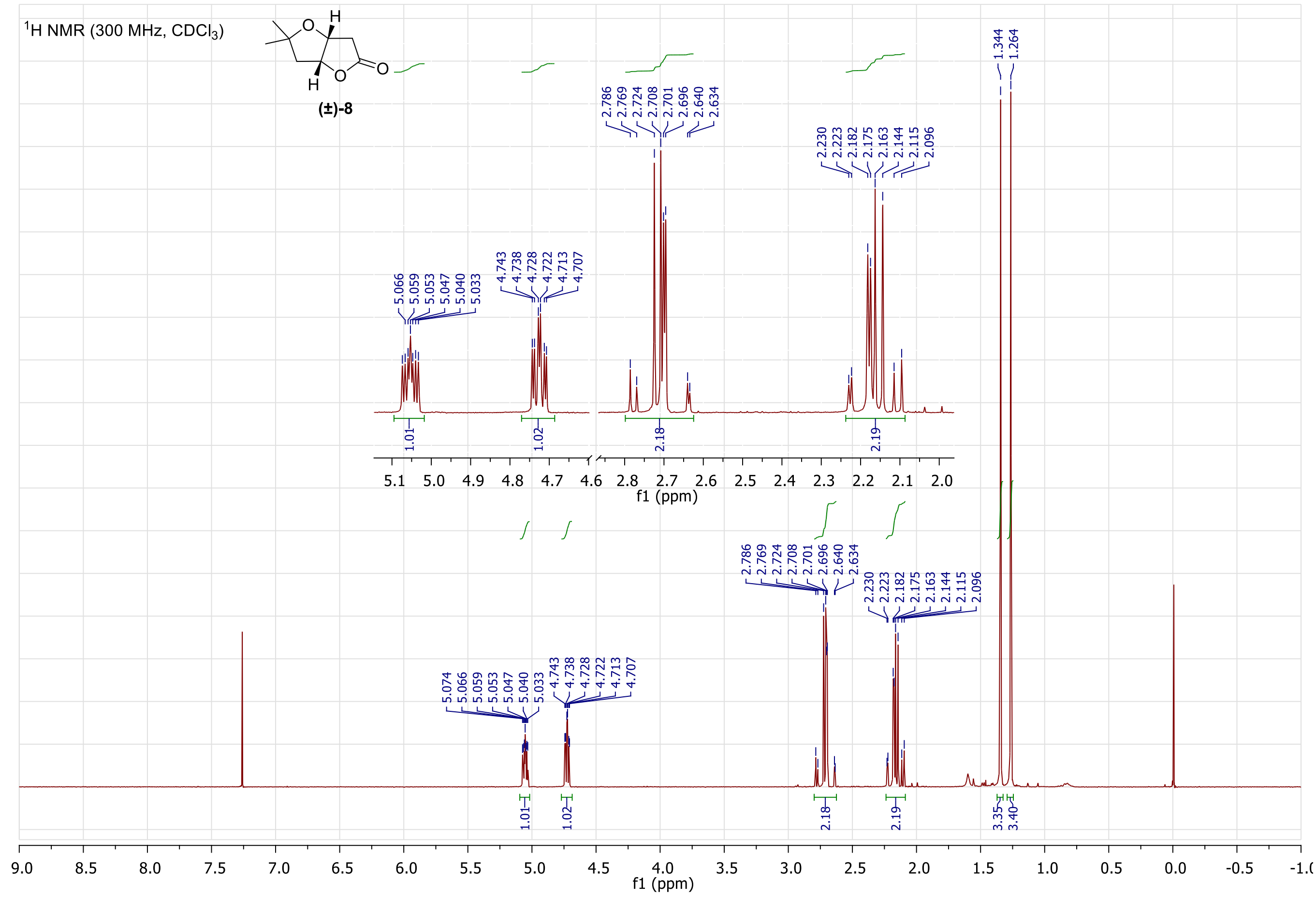




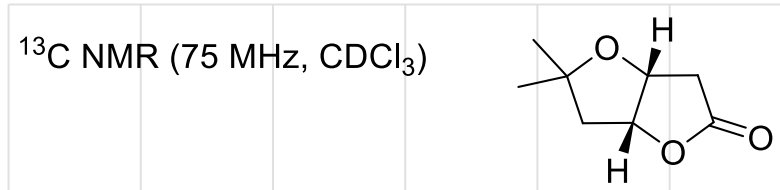

(士)-8

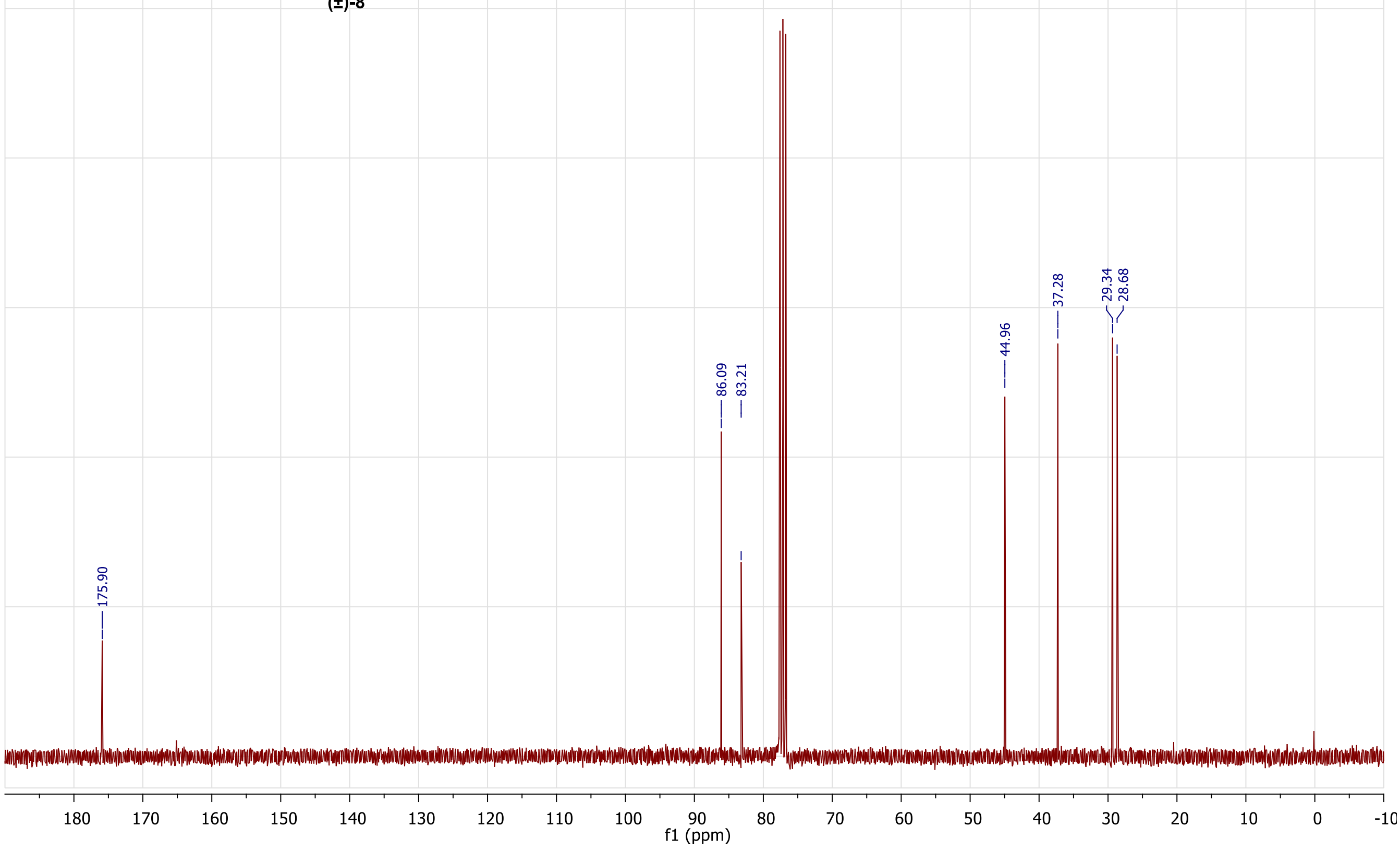




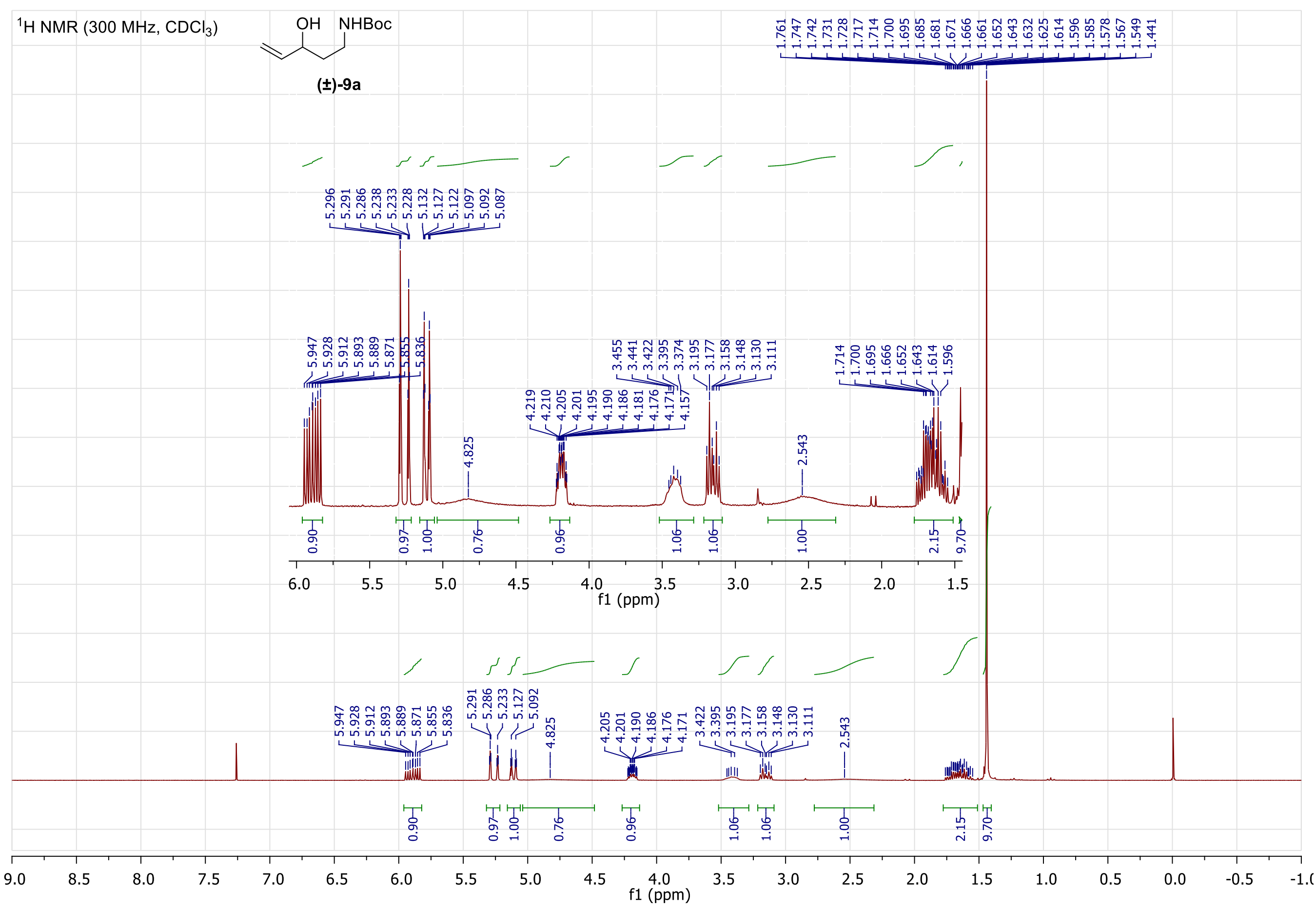




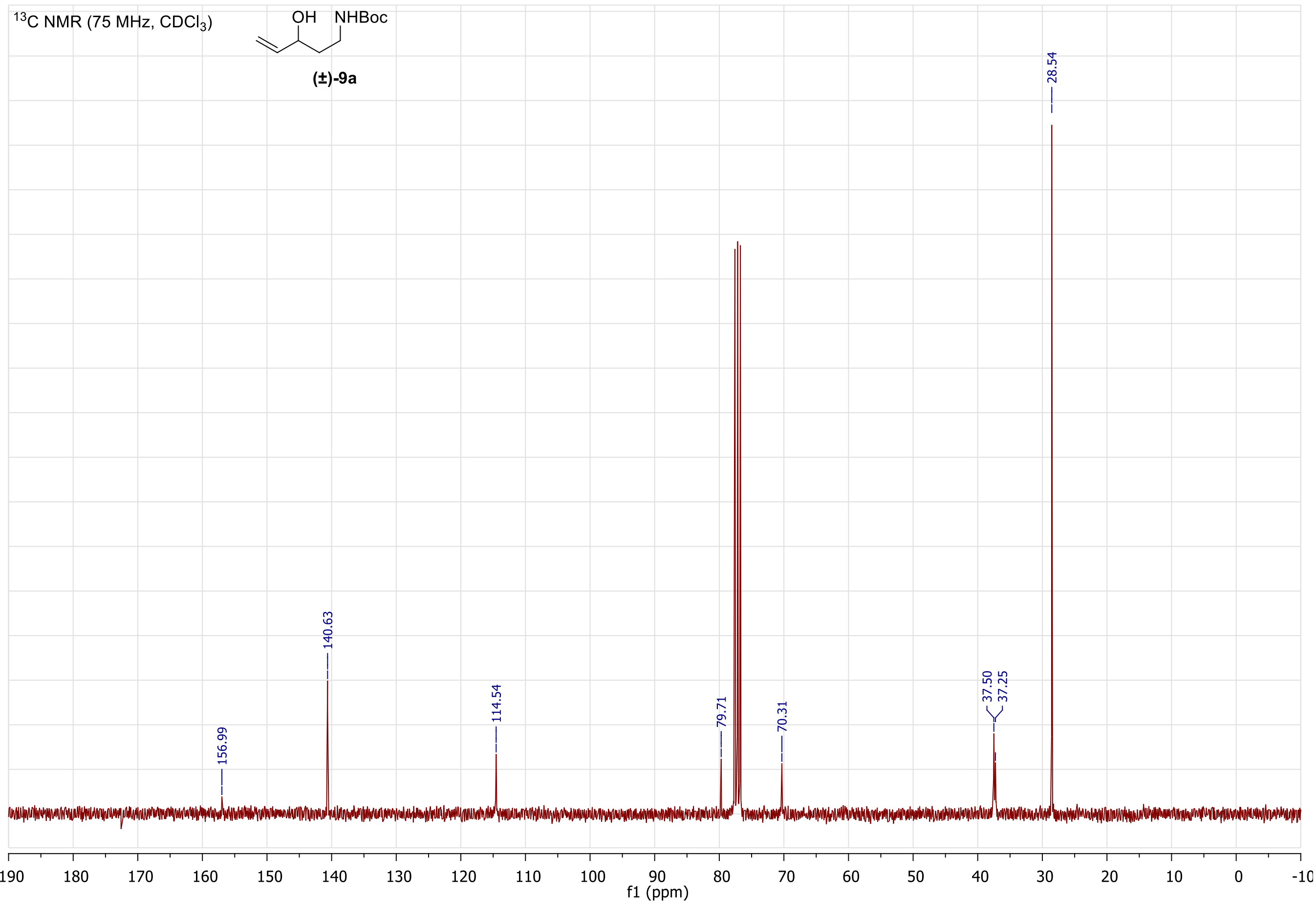




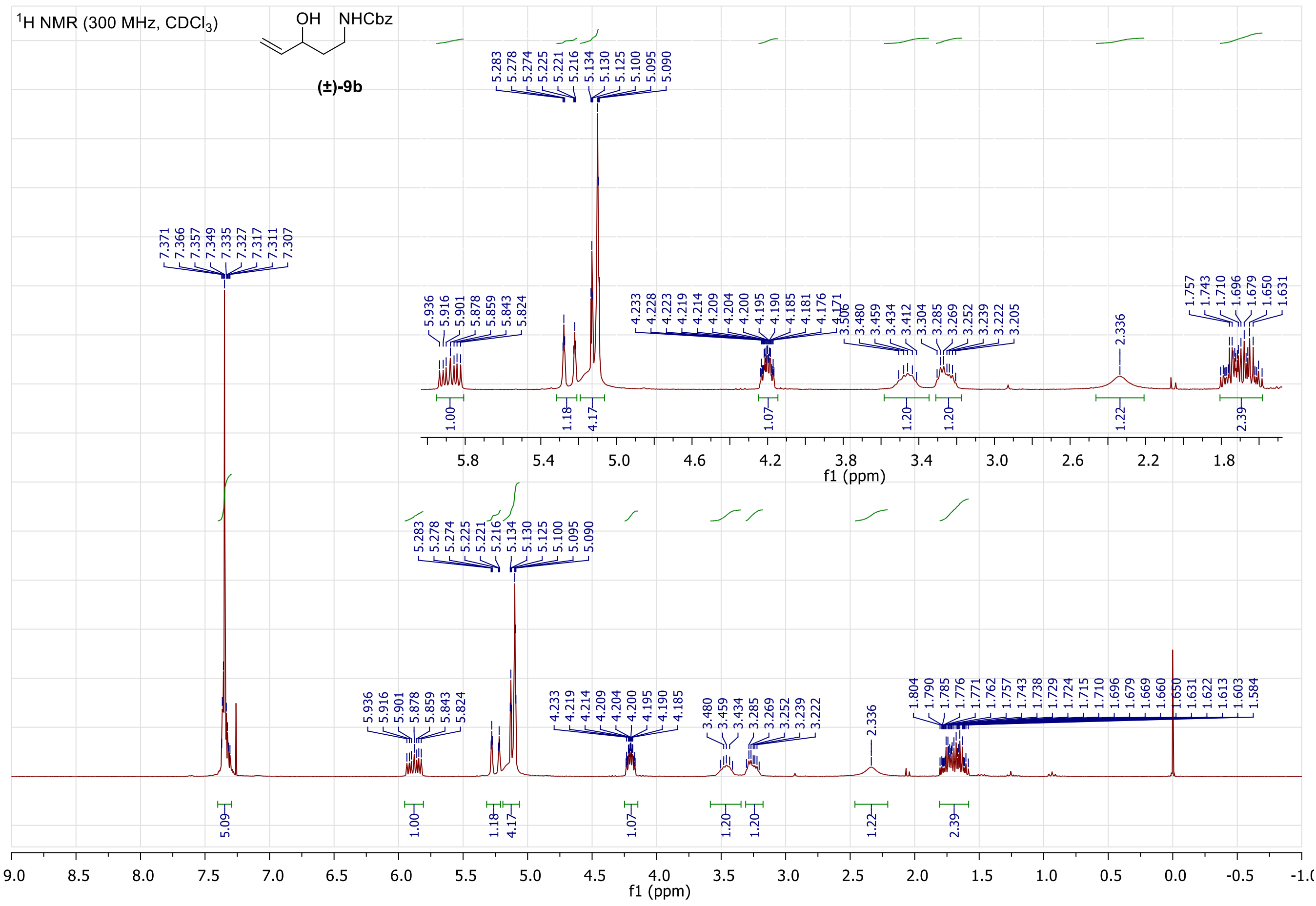




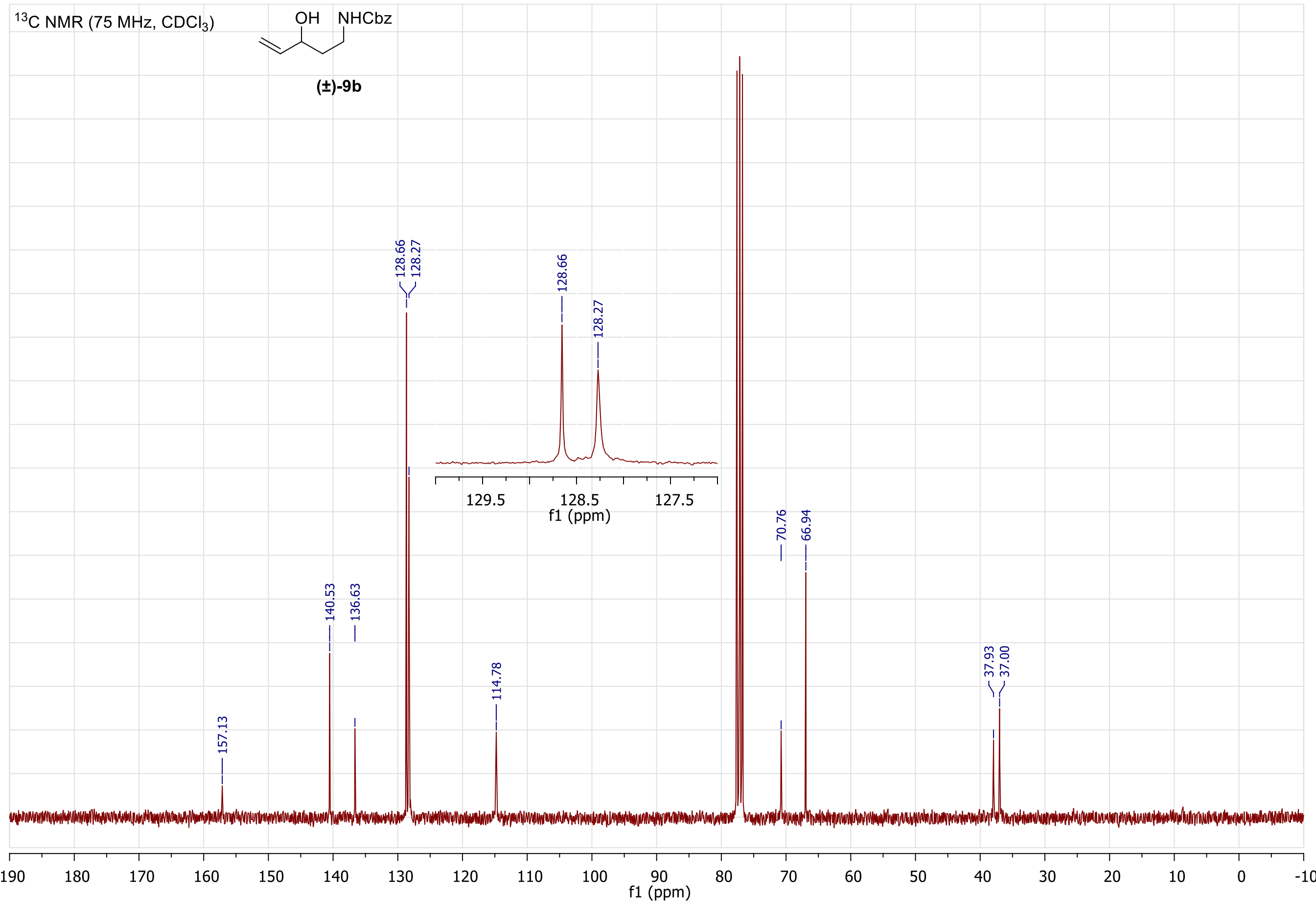




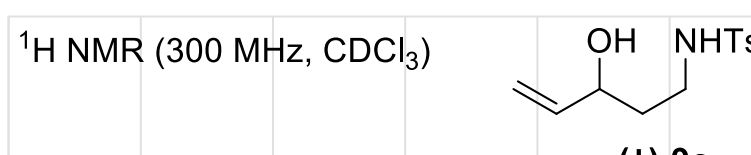

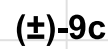

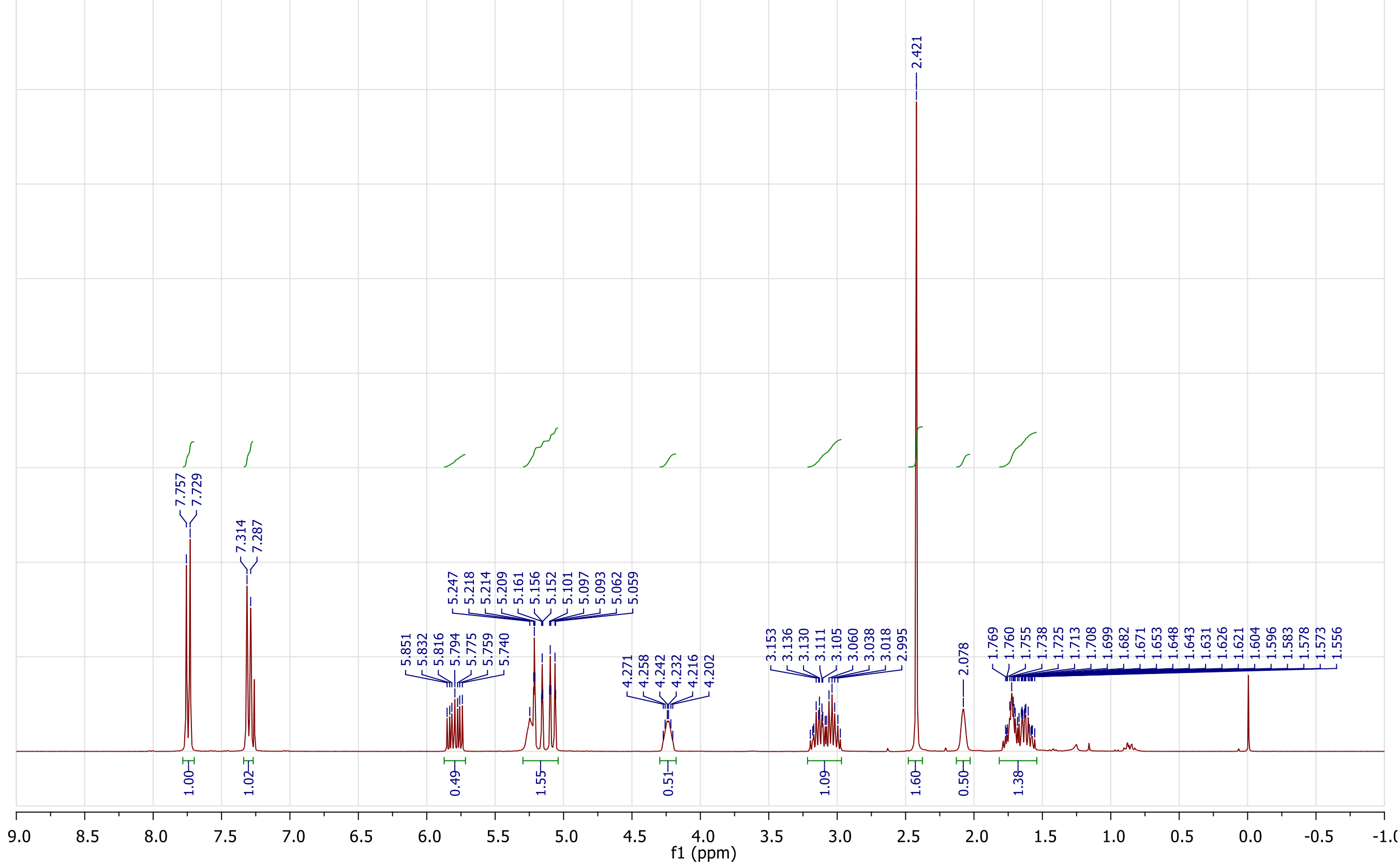




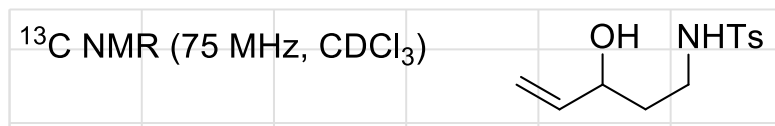

\section{(士)-9c}

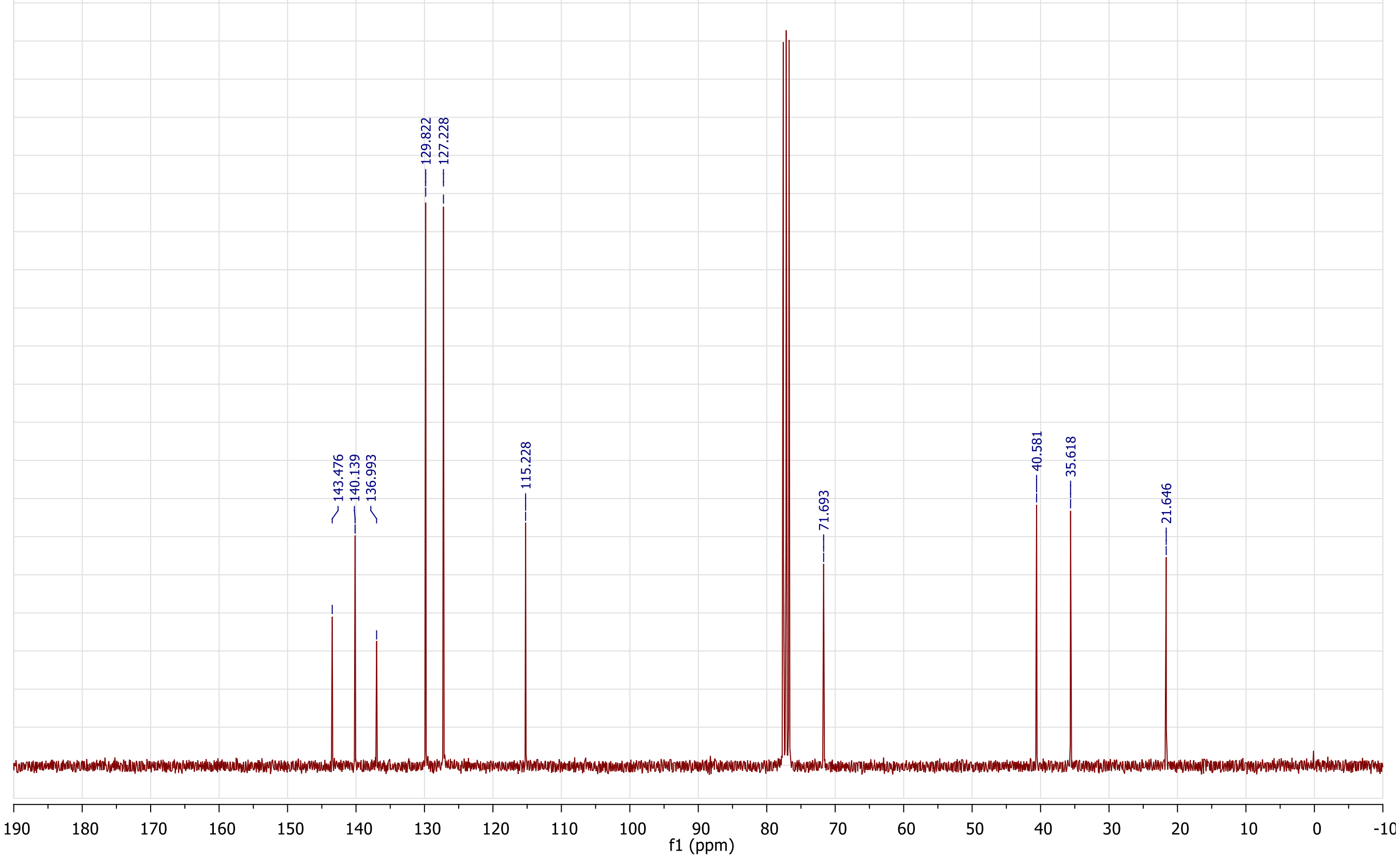



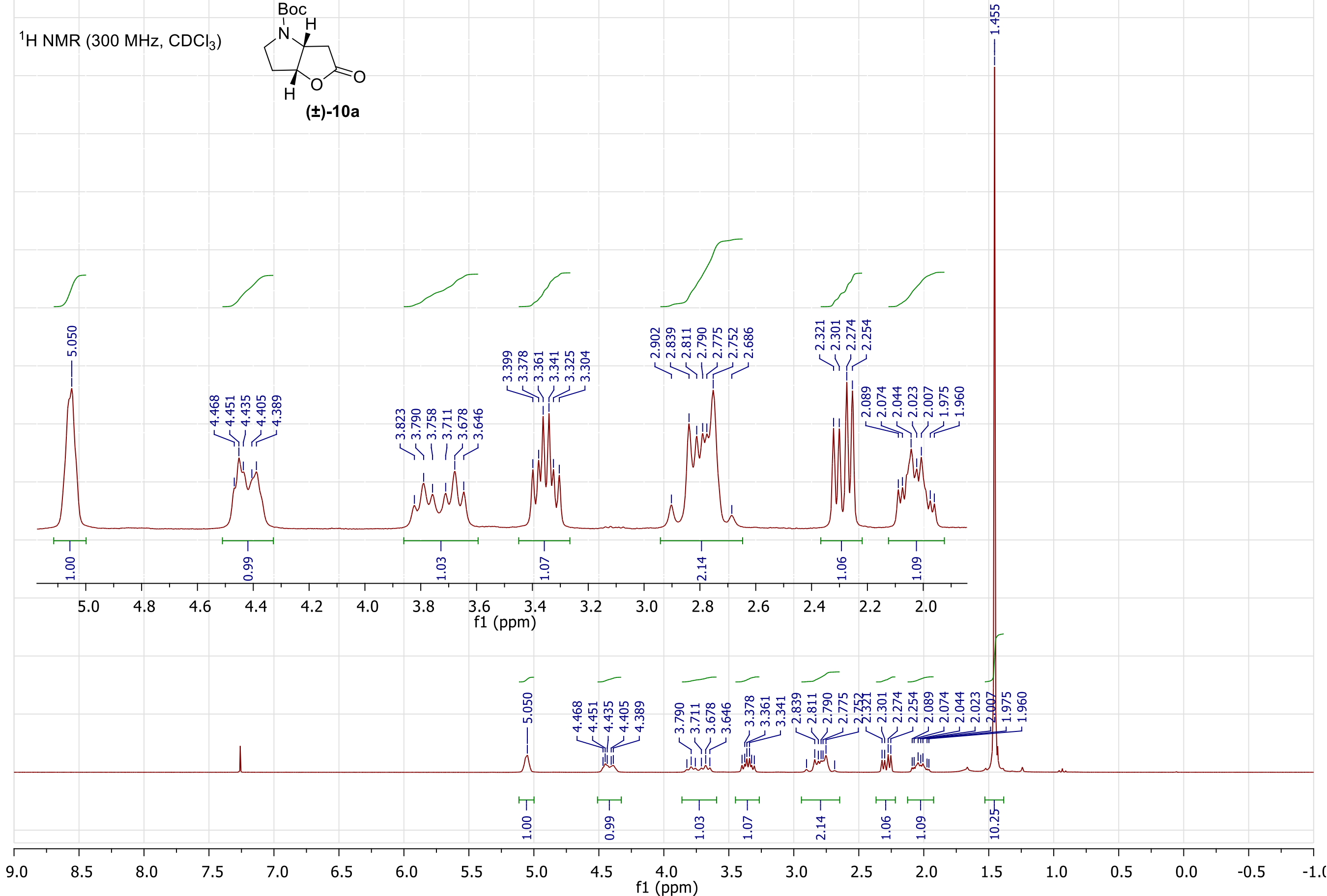

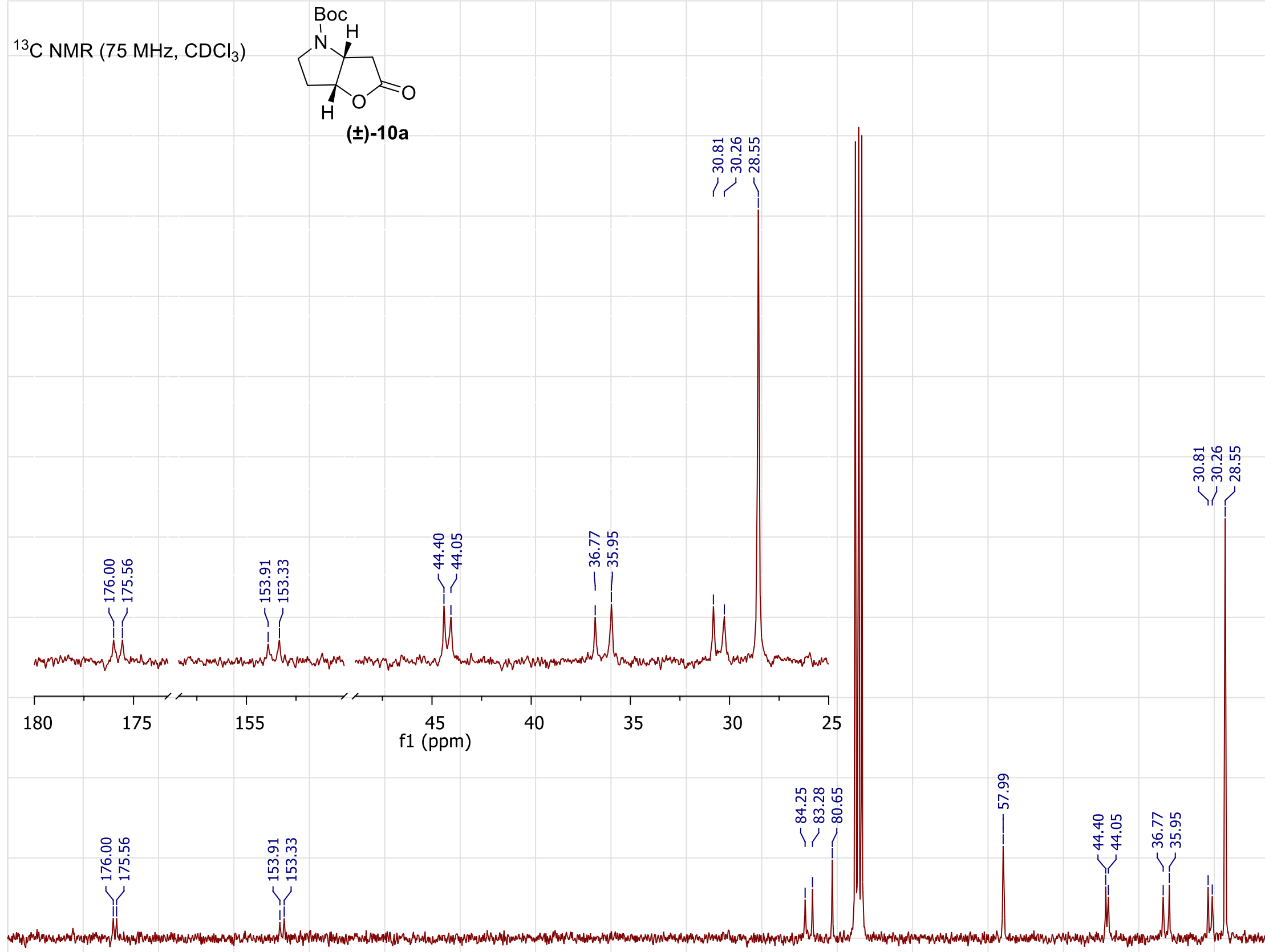

\begin{tabular}{|c|c|c|c|c|c|c|c|c|c|c|c|c|c|c|c|c|c|c|c|c|}
\hline Г & $T$ & 1 & $T$ & $T$ & $T$ & $T$ & $T$ & $T$ & $T$ & $T$ & 1 & $T$ & $T$ & 1 & $T$ & $T$ & $T$ & $T$ & $T$ & $\neg$ \\
\hline 190 & 180 & 170 & 160 & 150 & 140 & 130 & 120 & 110 & 100 & $\begin{array}{c}90 \\
\mathrm{f} 1(\mathrm{ppm})\end{array}$ & 80 & 70 & 60 & 50 & 40 & 30 & 20 & 10 & 0 & $-1 C$ \\
\hline
\end{tabular}


${ }^{1} \mathrm{H}$ NMR $\left(300 \mathrm{MHz}, \mathrm{CDCl}_{3}\right.$ )
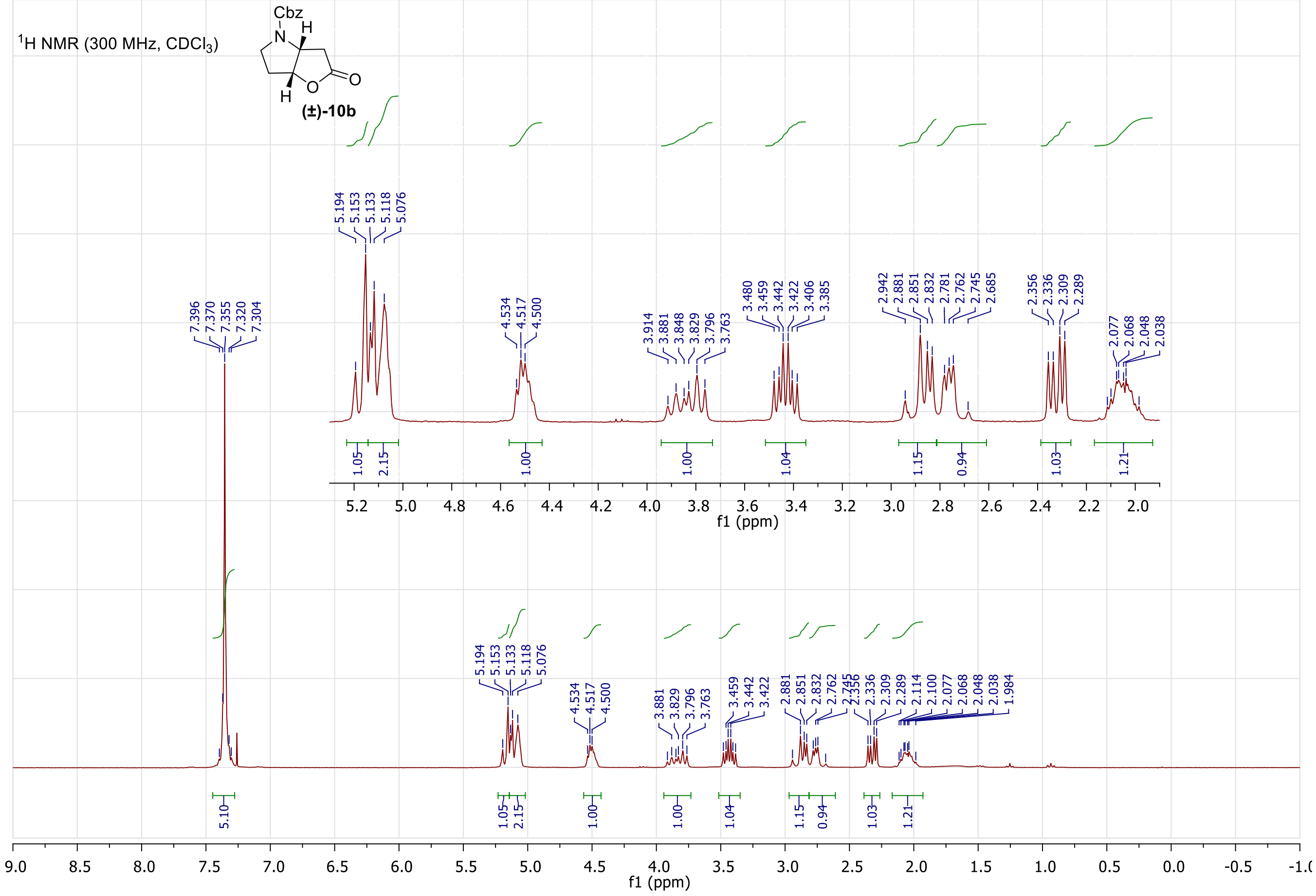
${ }^{13} \mathrm{C} \mathrm{NMR}\left(75 \mathrm{MHz}, \mathrm{CDCl}_{3}\right)$

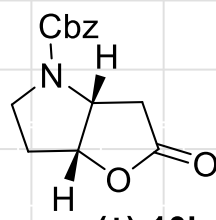

(士)-10b
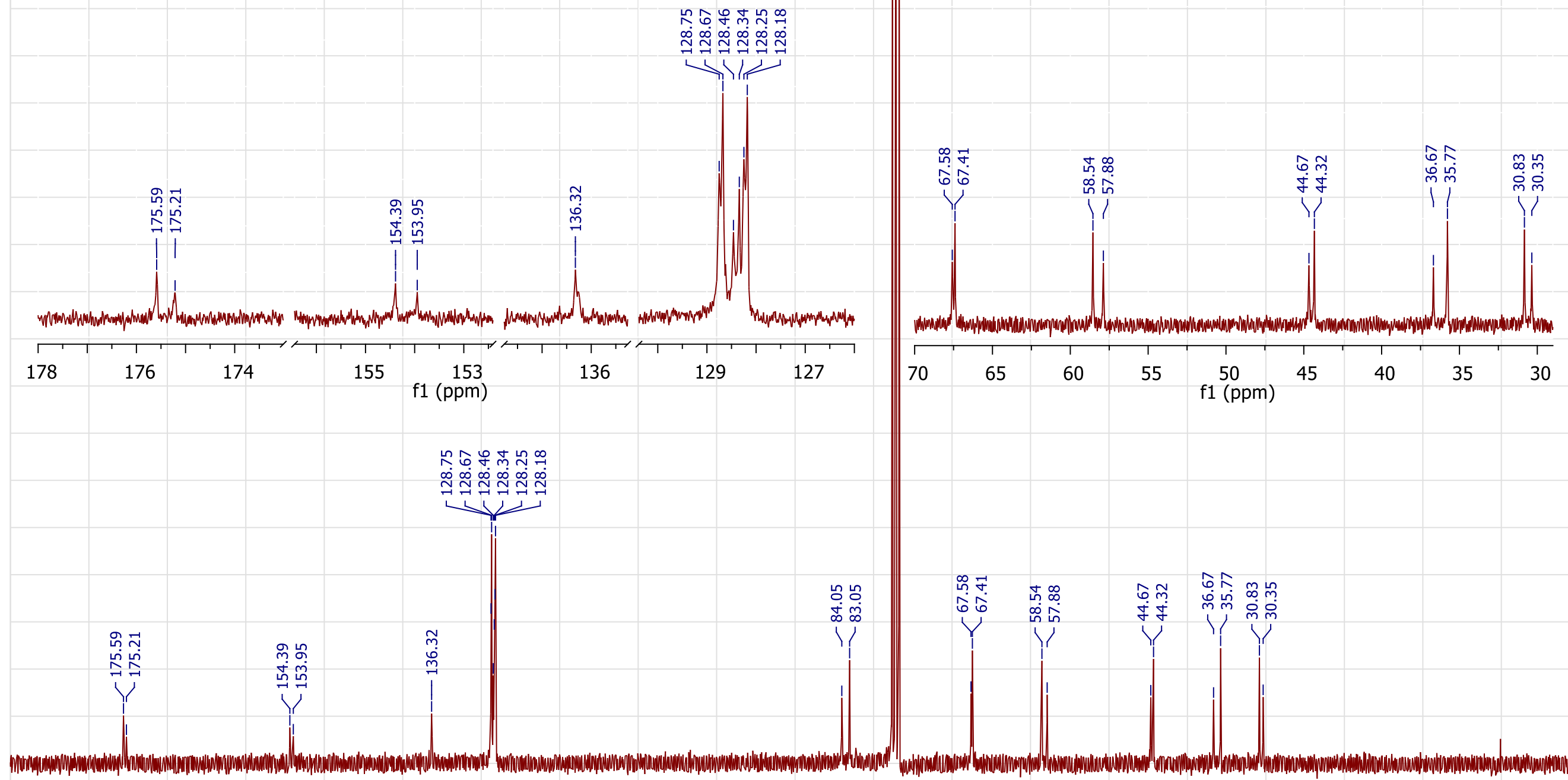

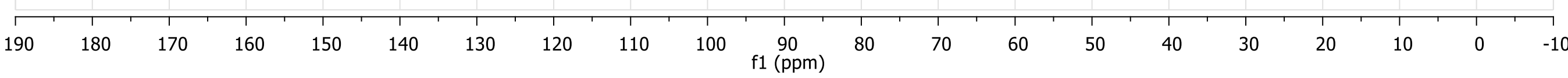




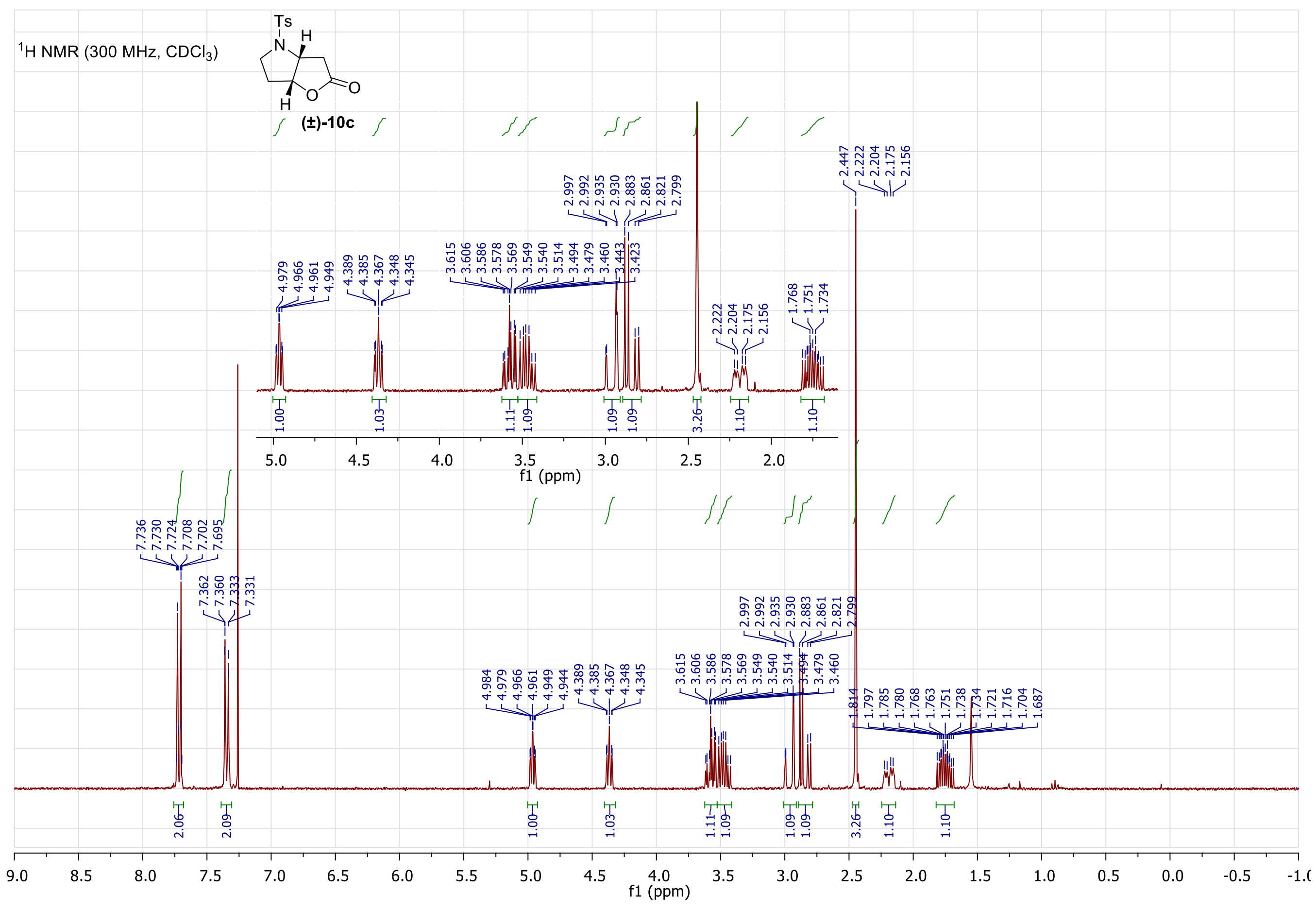




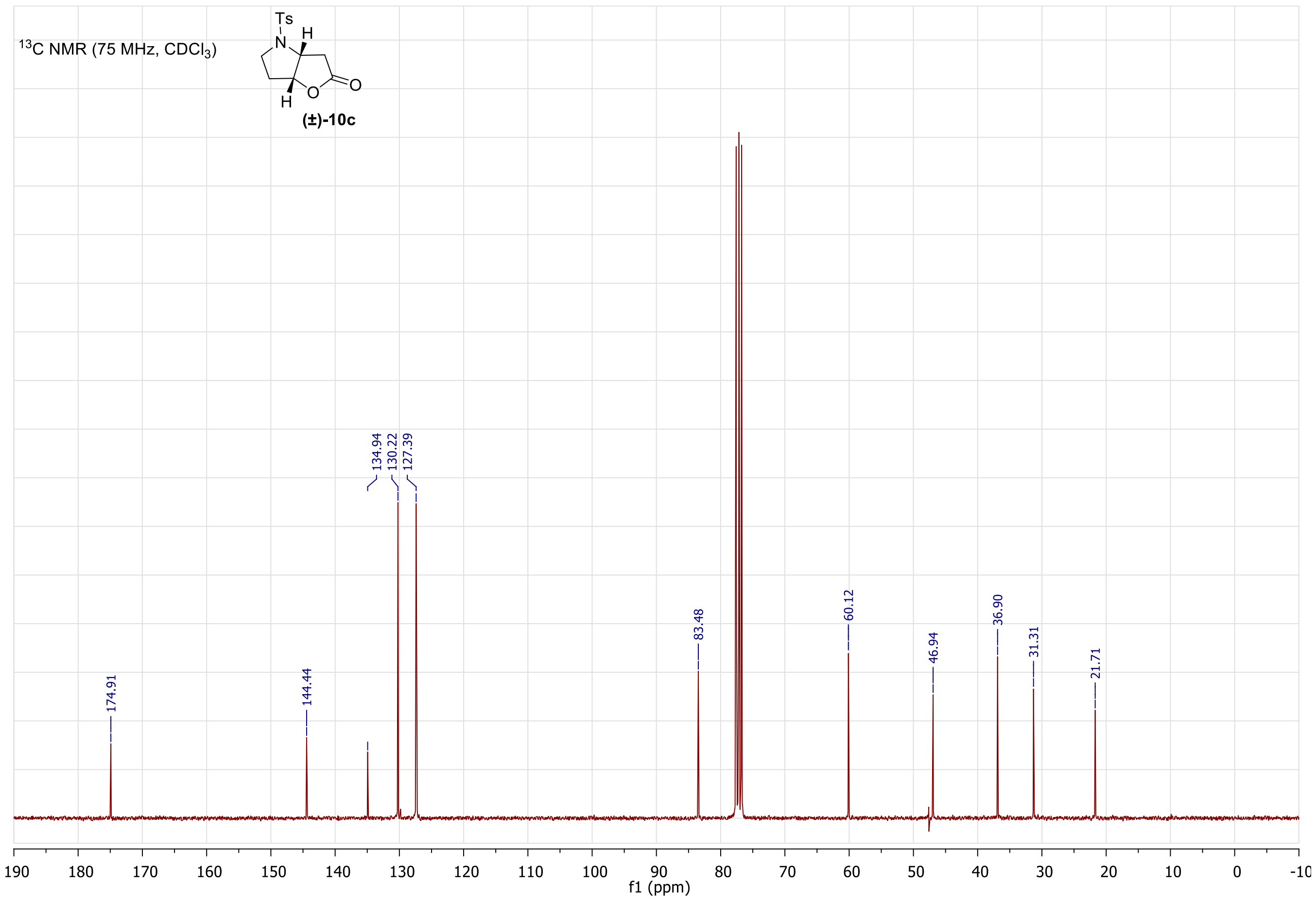




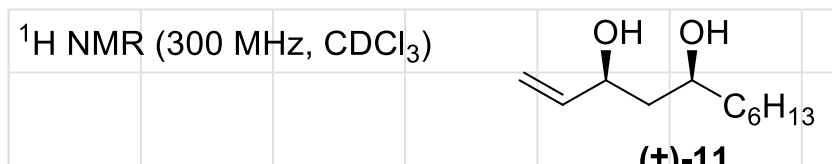

(土)-11

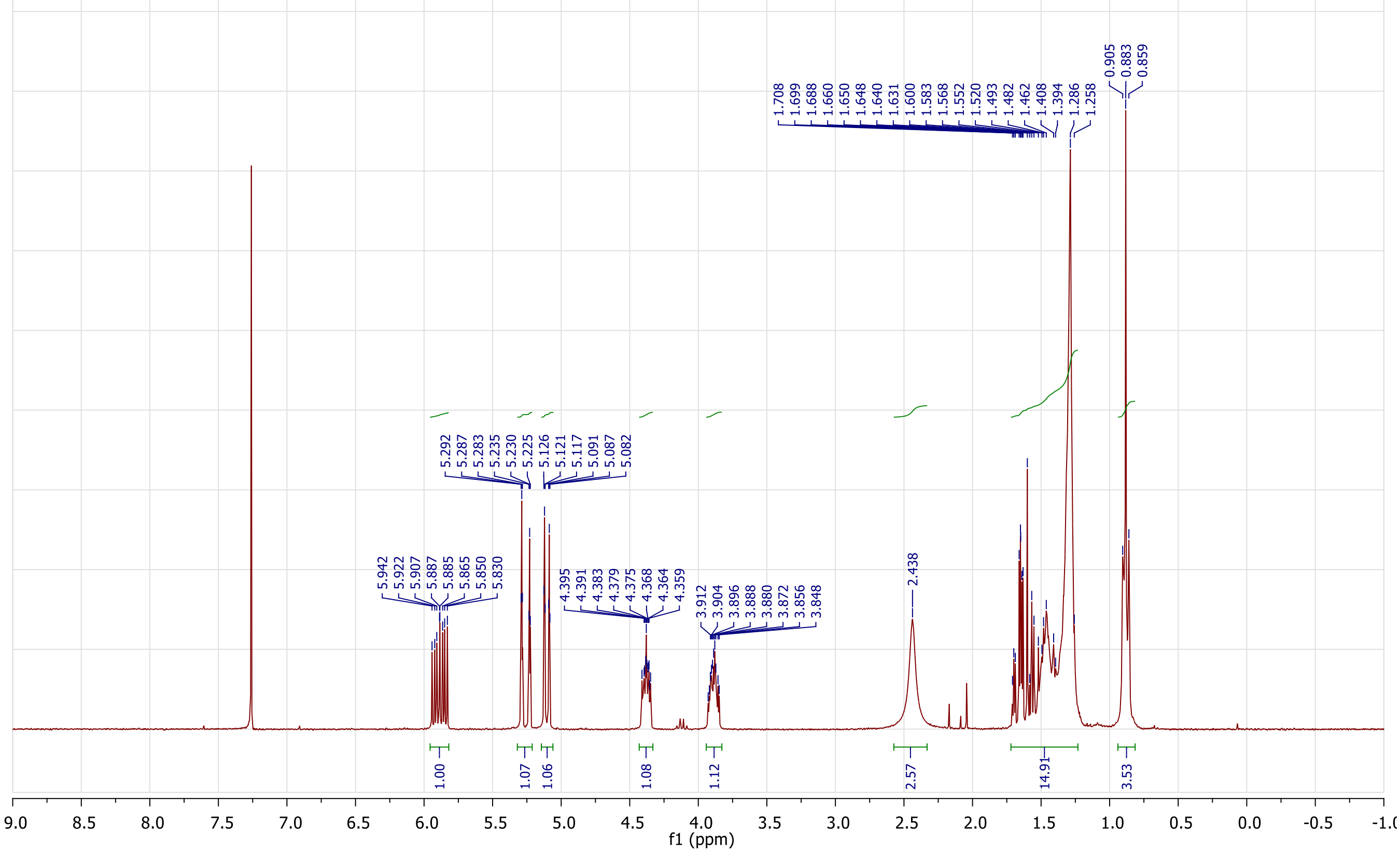


${ }^{13} \mathrm{C} \mathrm{NMR}\left(75 \mathrm{MHz}, \mathrm{CDCl}_{3}\right)$

(士)-11

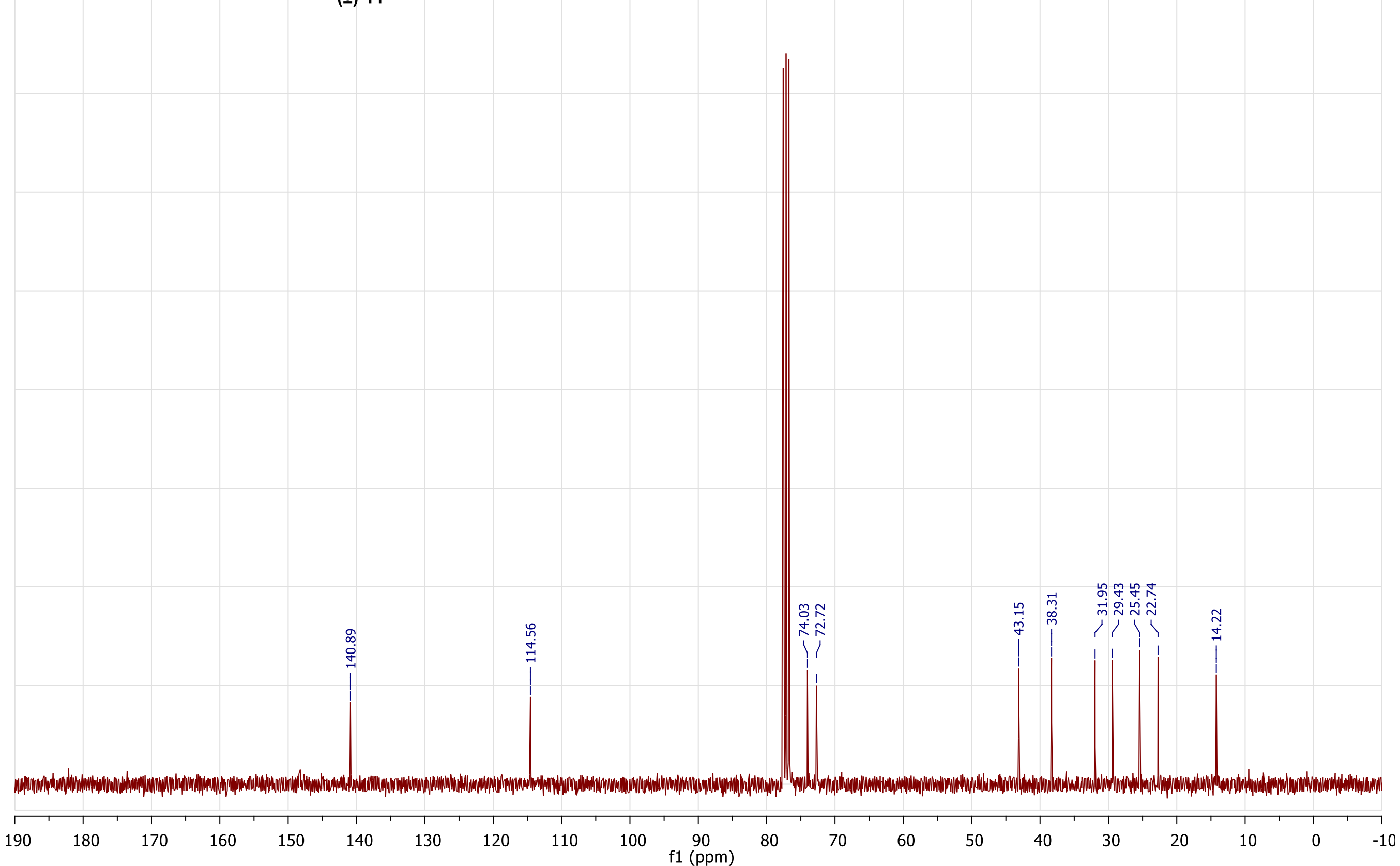




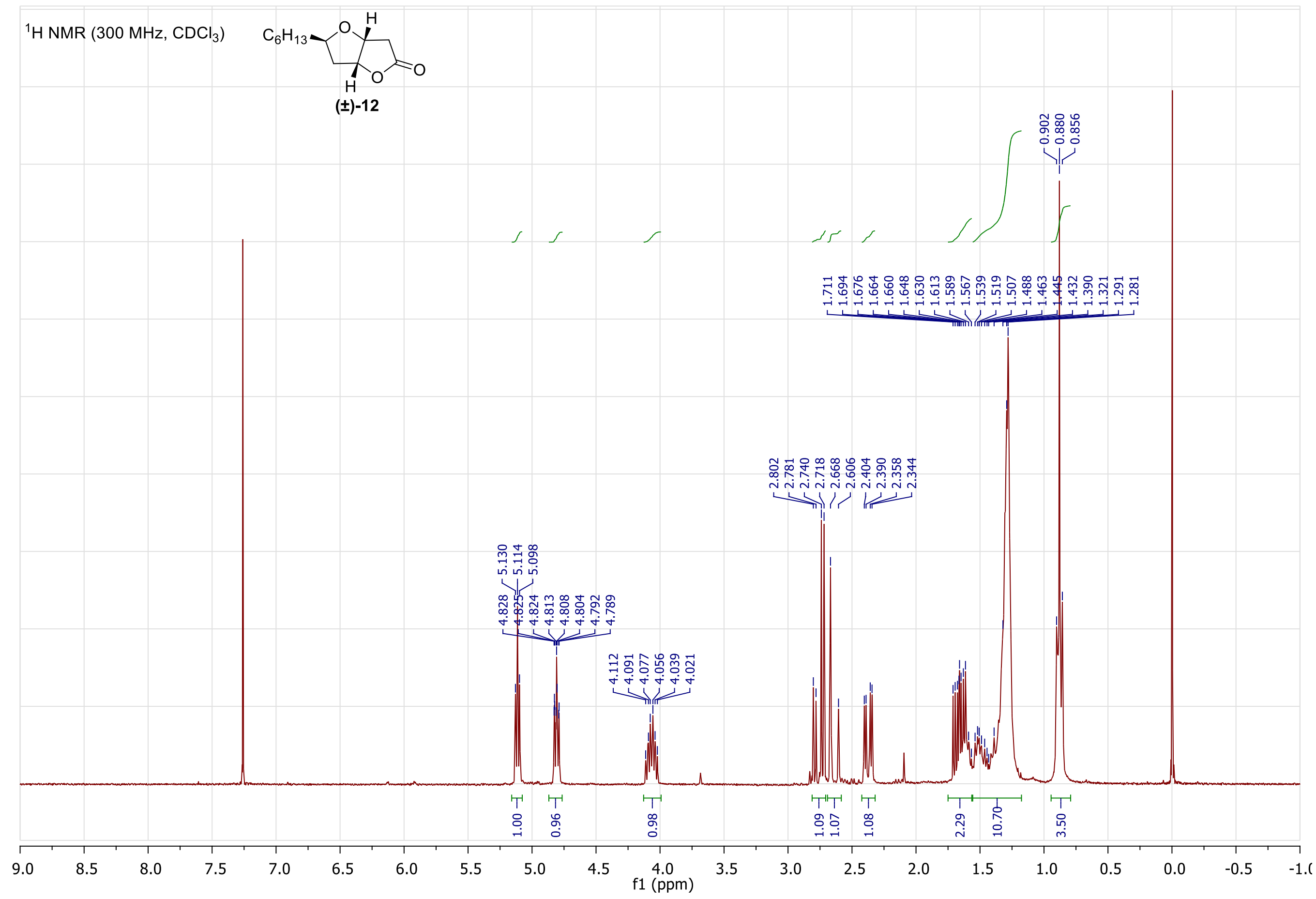



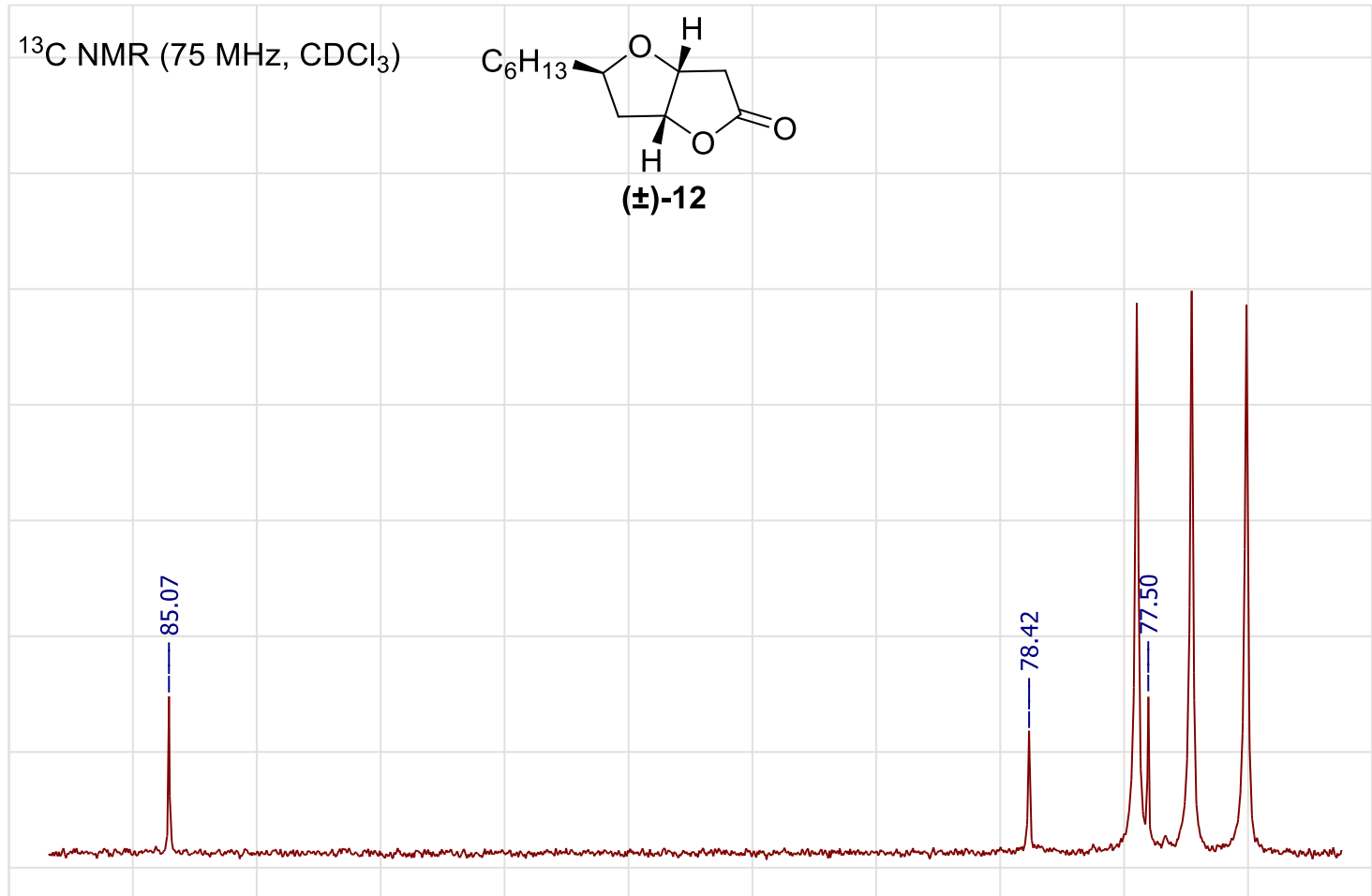

86

85

84

83

82

81
f1

80

79

78

77
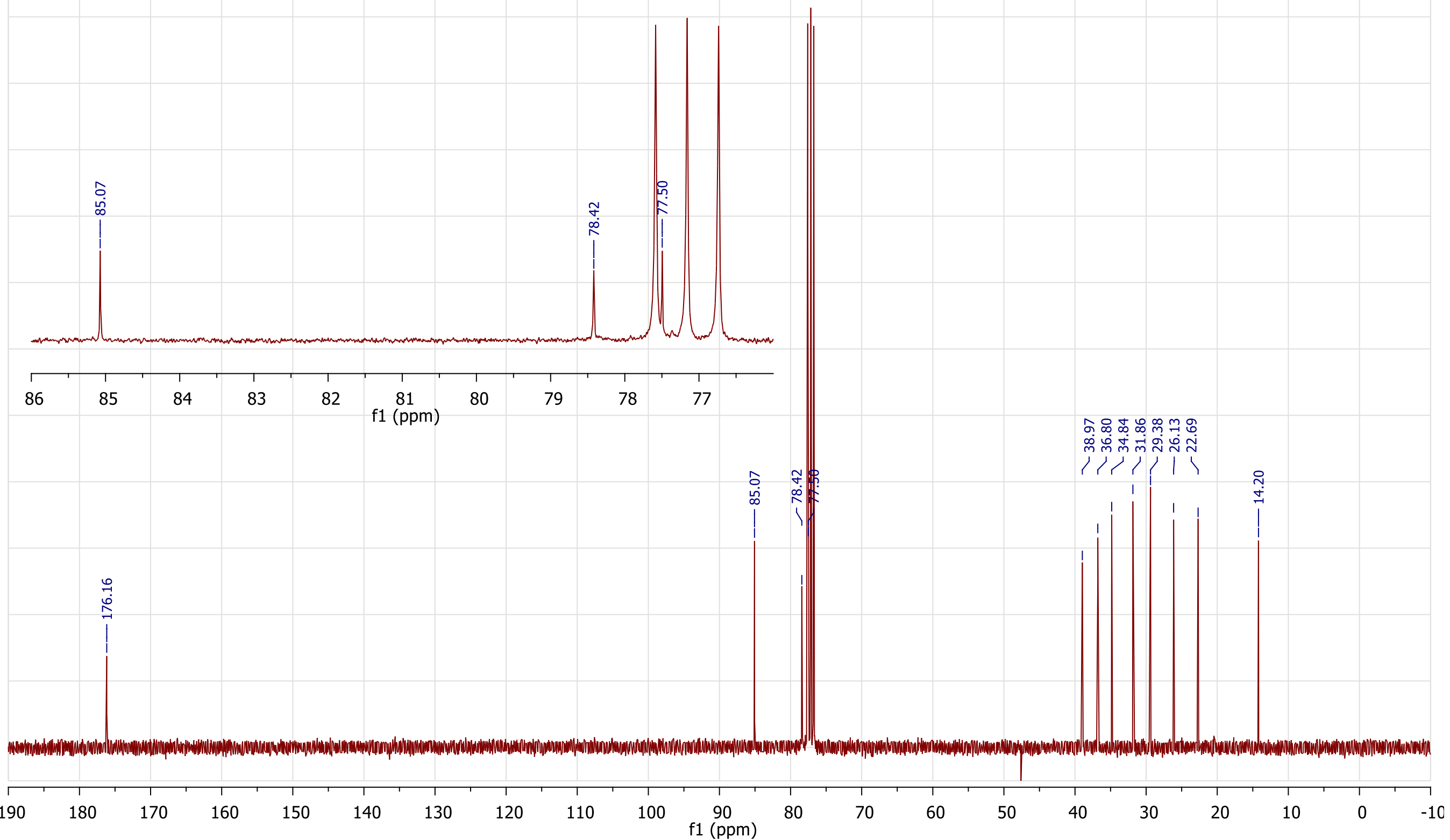


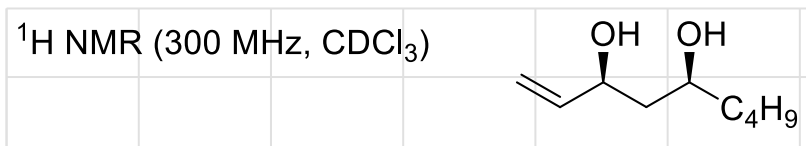

(士)-13

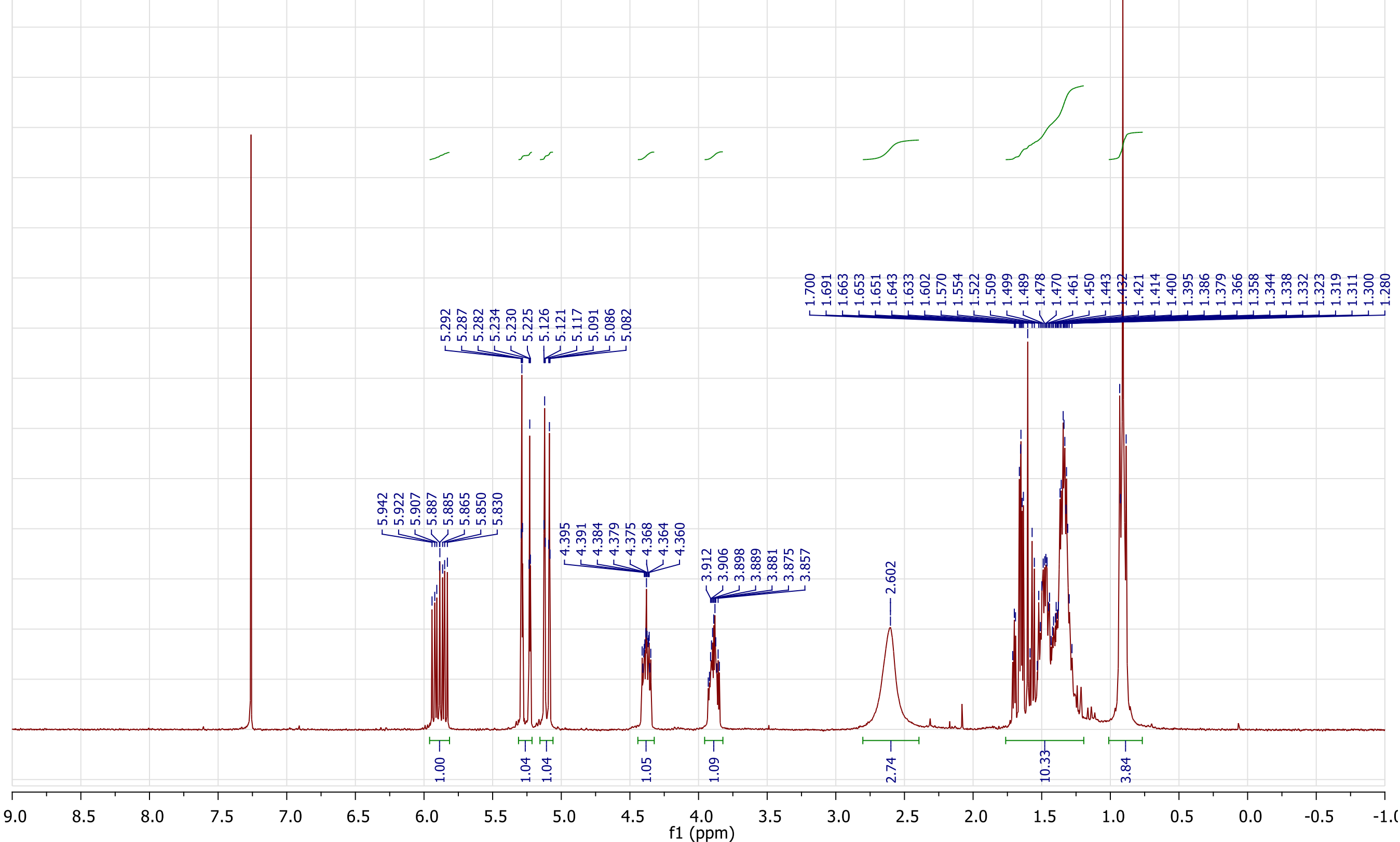




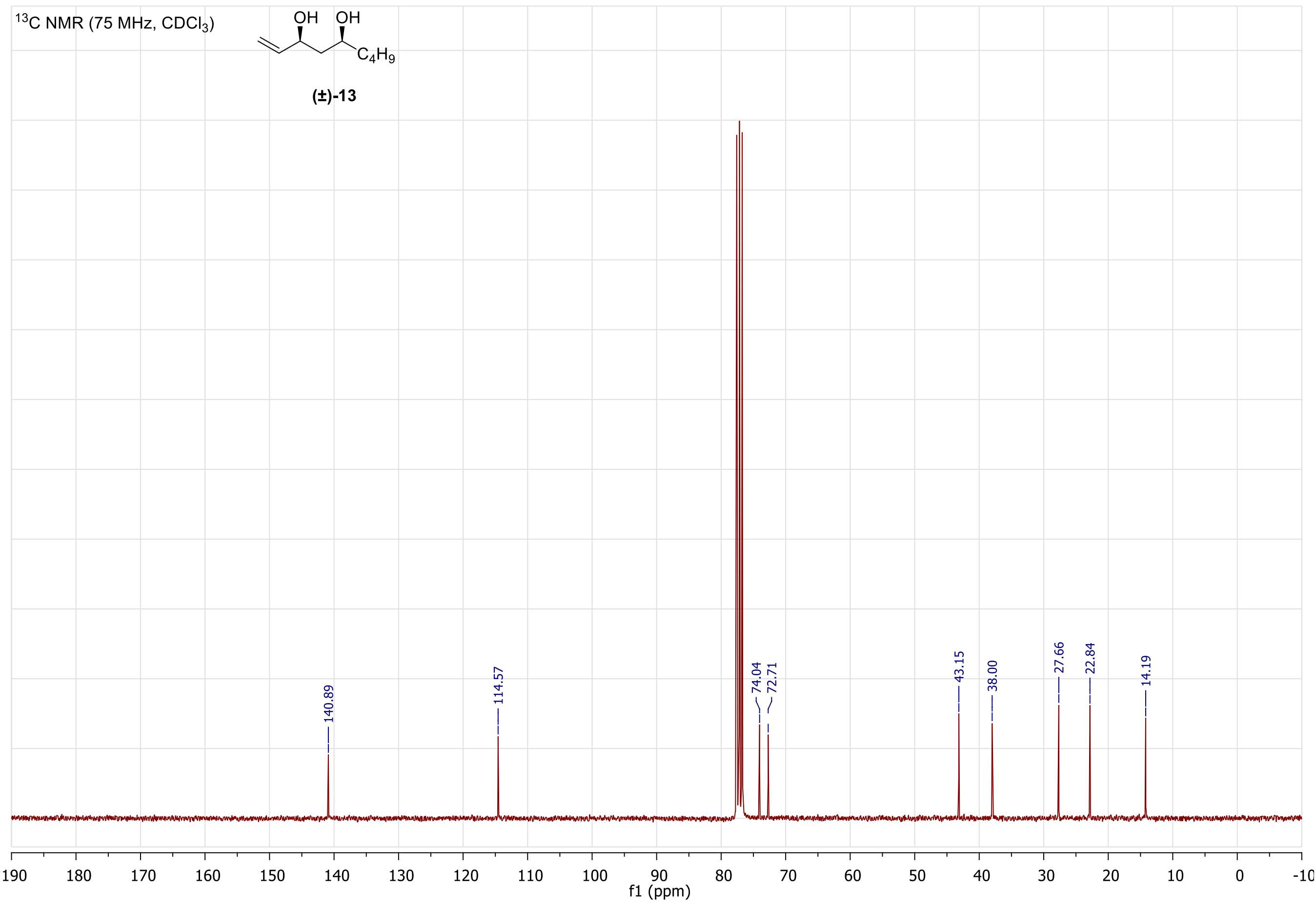




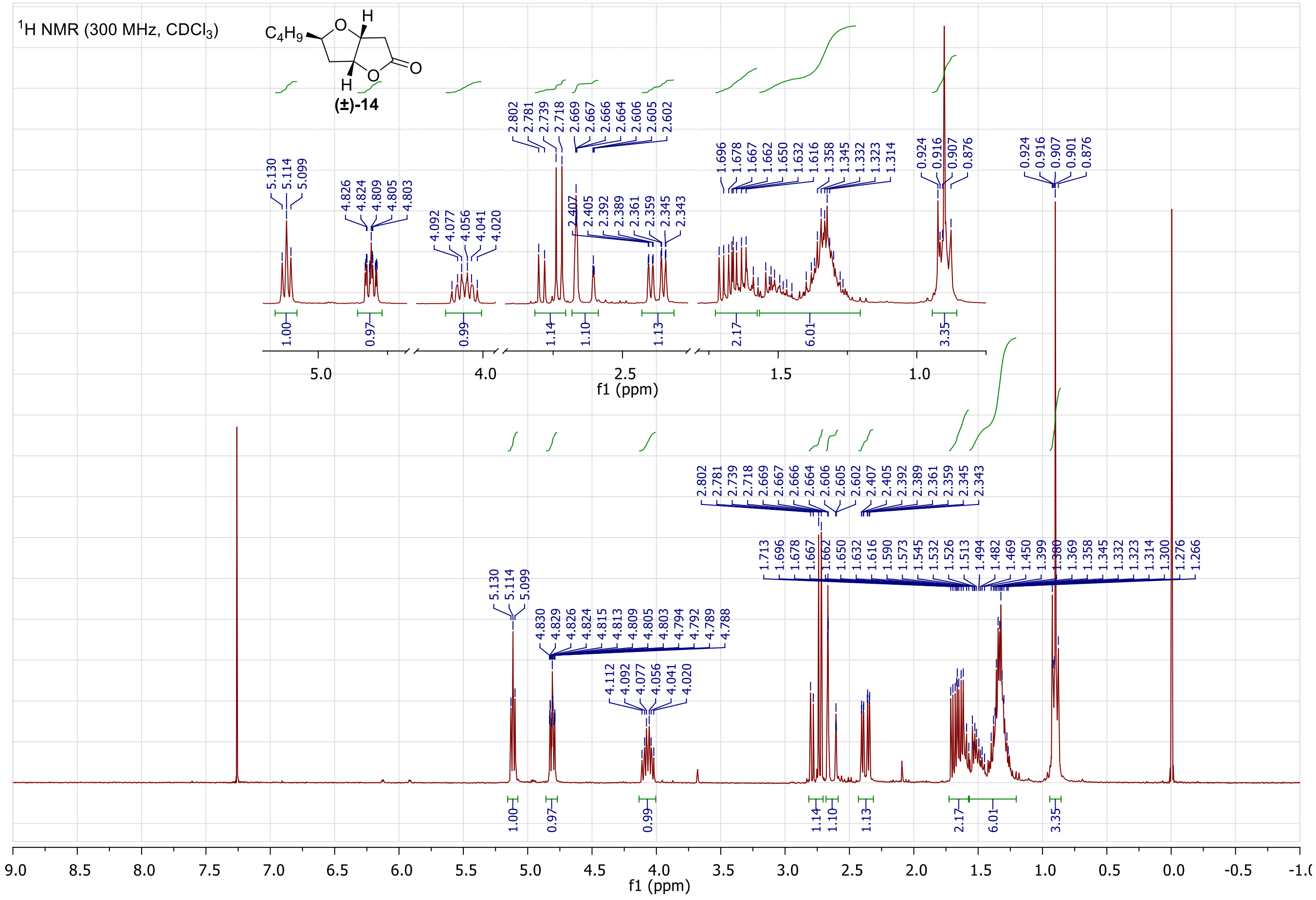




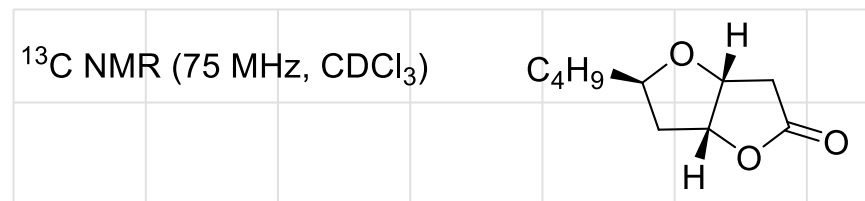

$( \pm)-14$

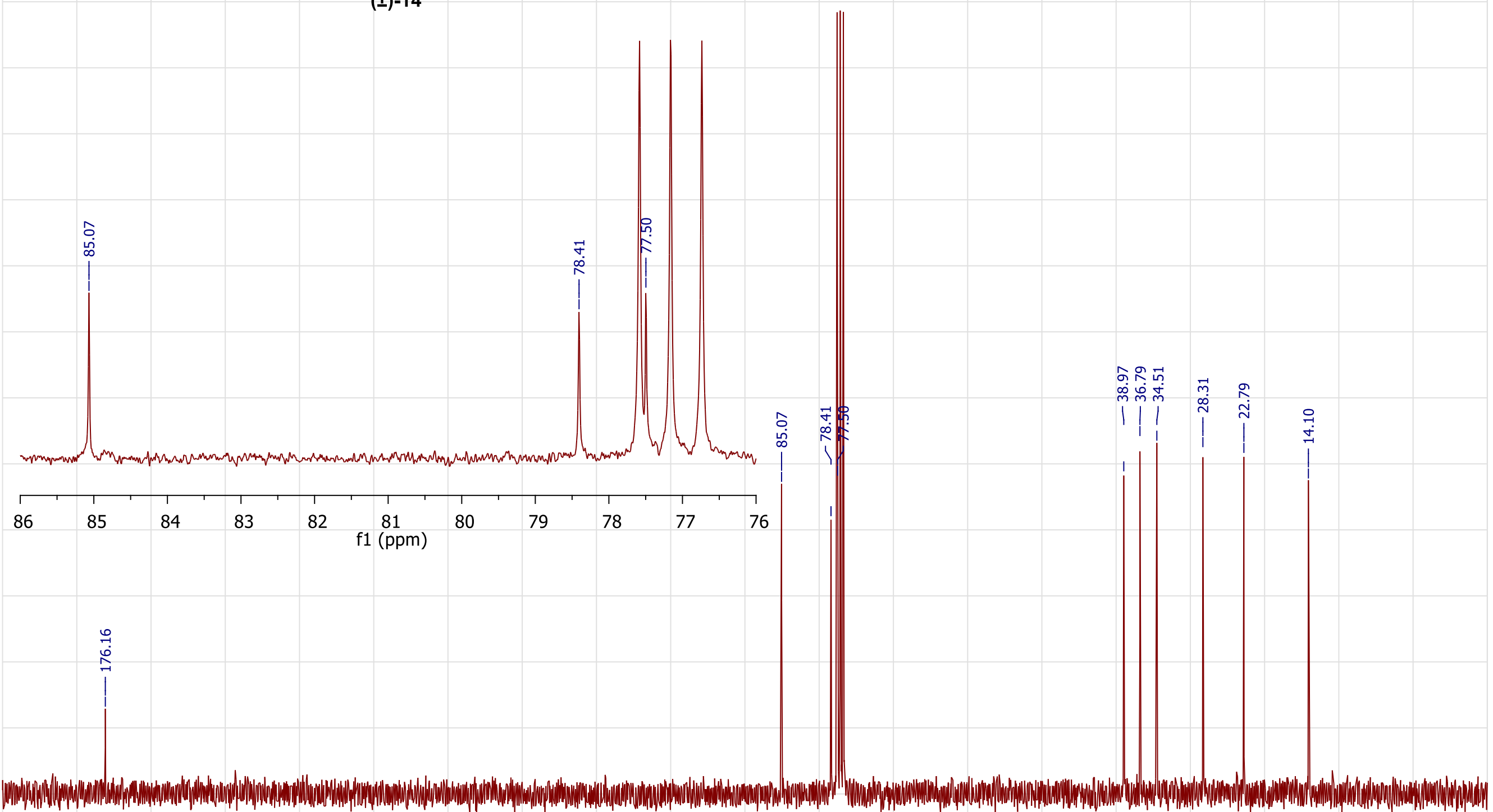

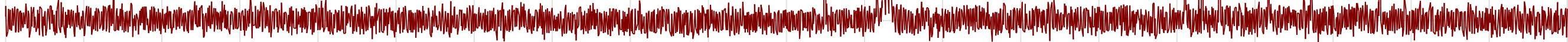




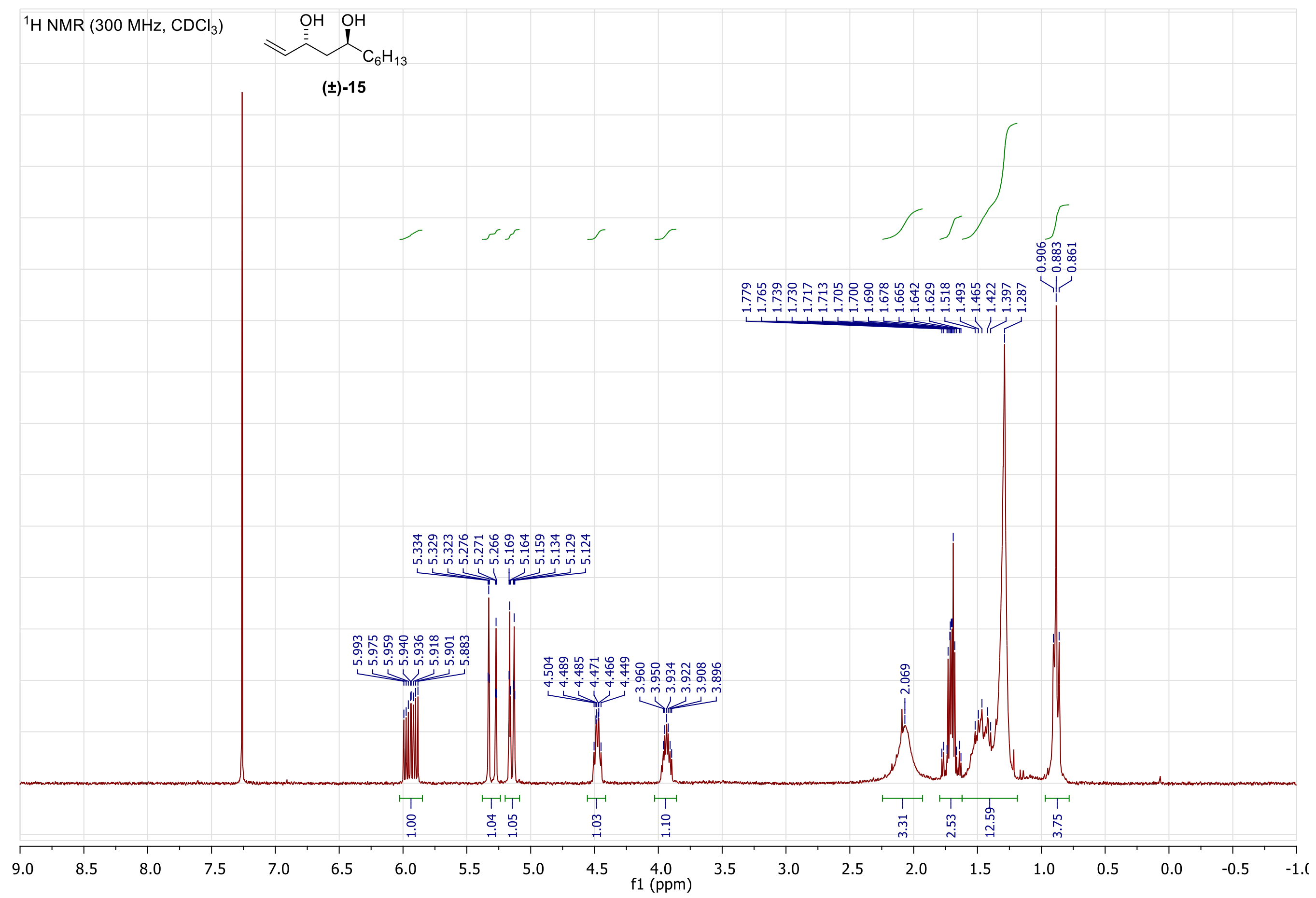




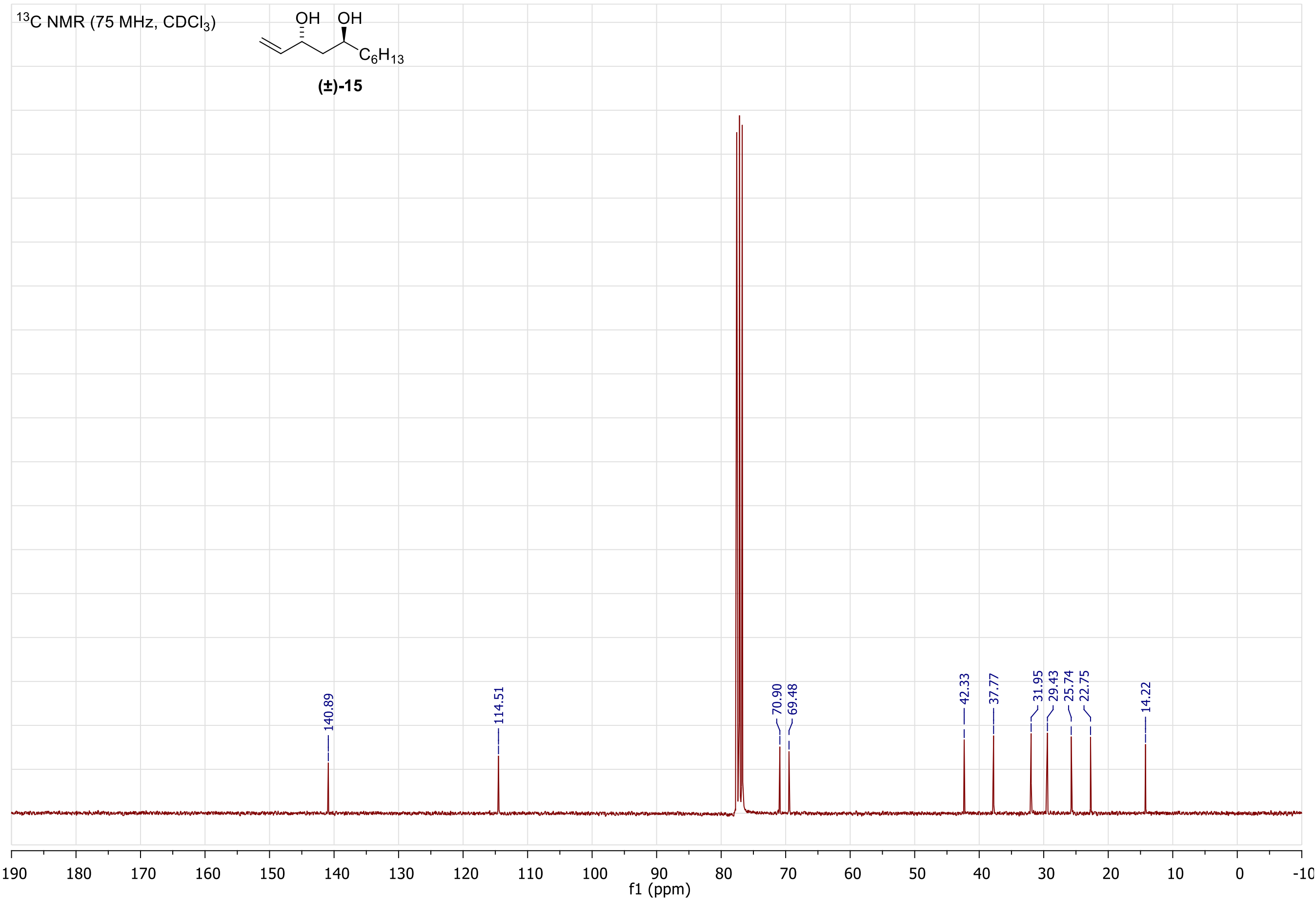




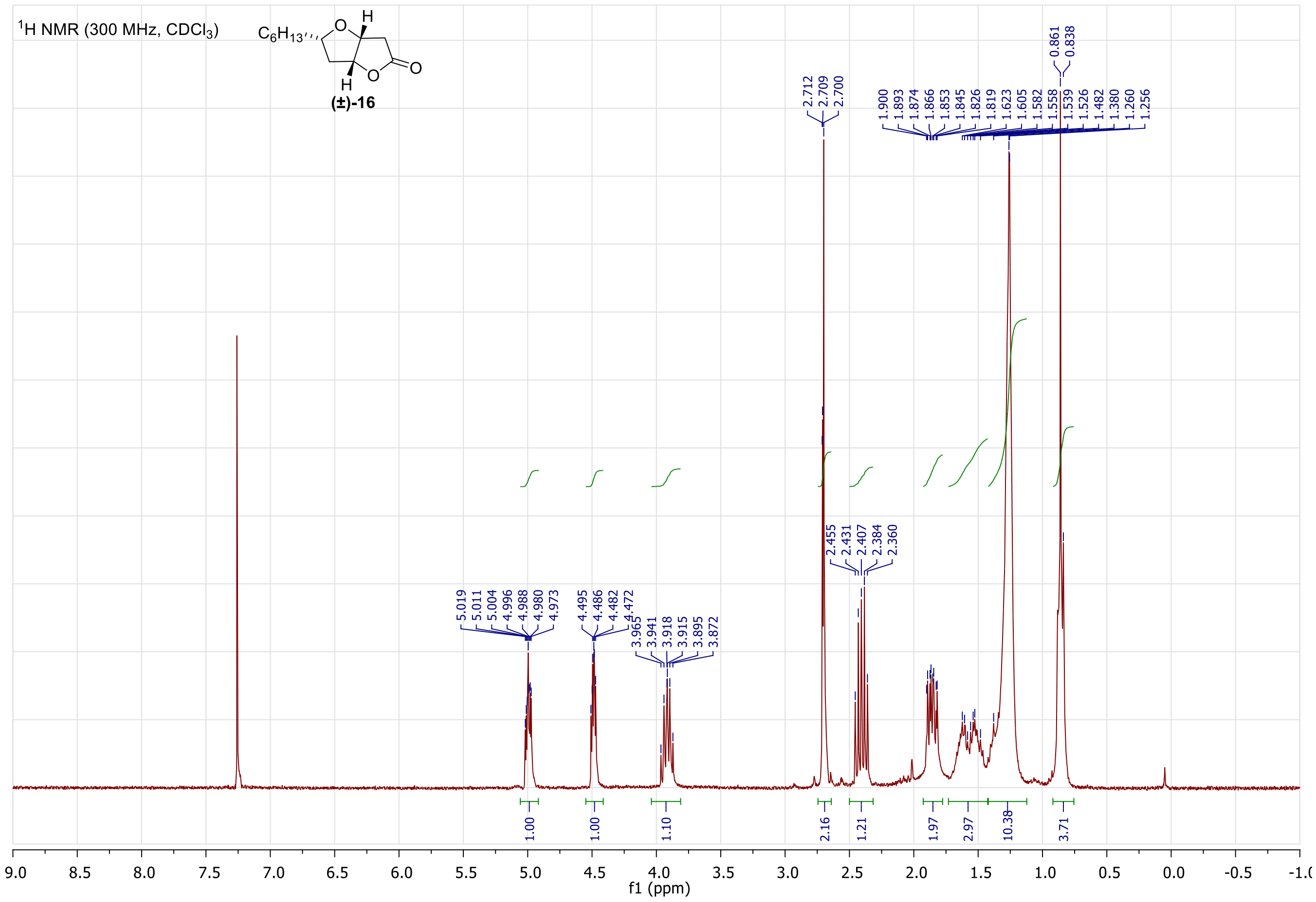




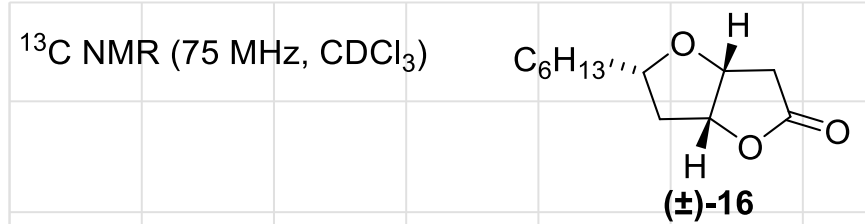

กุ

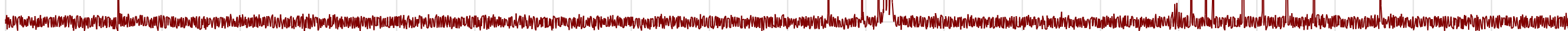

\begin{tabular}{|c|c|c|c|c|c|c|c|c|c|c|c|c|c|c|c|c|c|c|c|}
\hline \multirow{2}{*}{$\Gamma$} & $T$ & $T$ & $T$ & $T$ & $T$ & $T$ & $T$ & $T$ & $T$ & $T$ & $T$ & $T$ & 1 & T & $T$ & $T$ & $T$ & $T$ & $T$ \\
\hline & 180 & 170 & 160 & 150 & 140 & 130 & 120 & 110 & 100 & $\begin{array}{c}90 \\
\mathrm{f} 1(\mathrm{ppm})\end{array}$ & 80 & 70 & 60 & 50 & 40 & 30 & 20 & 10 & 0 \\
\hline
\end{tabular}




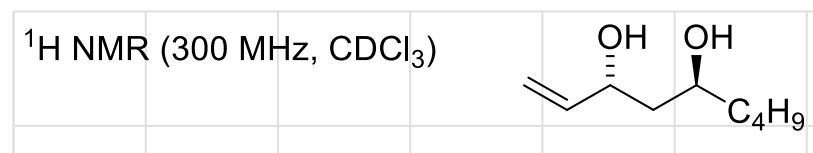

$( \pm)-17$

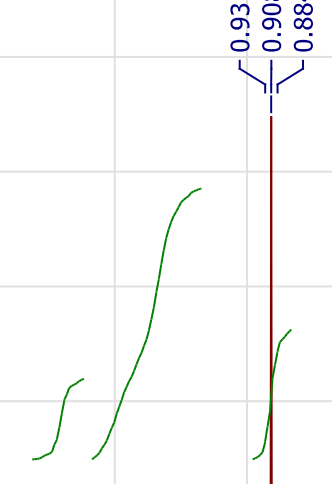

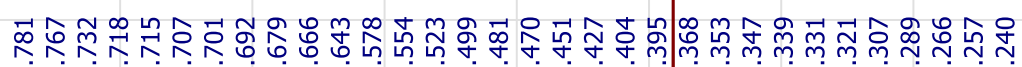

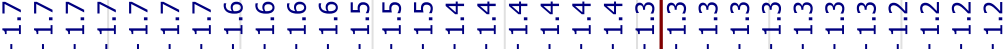

舟

政
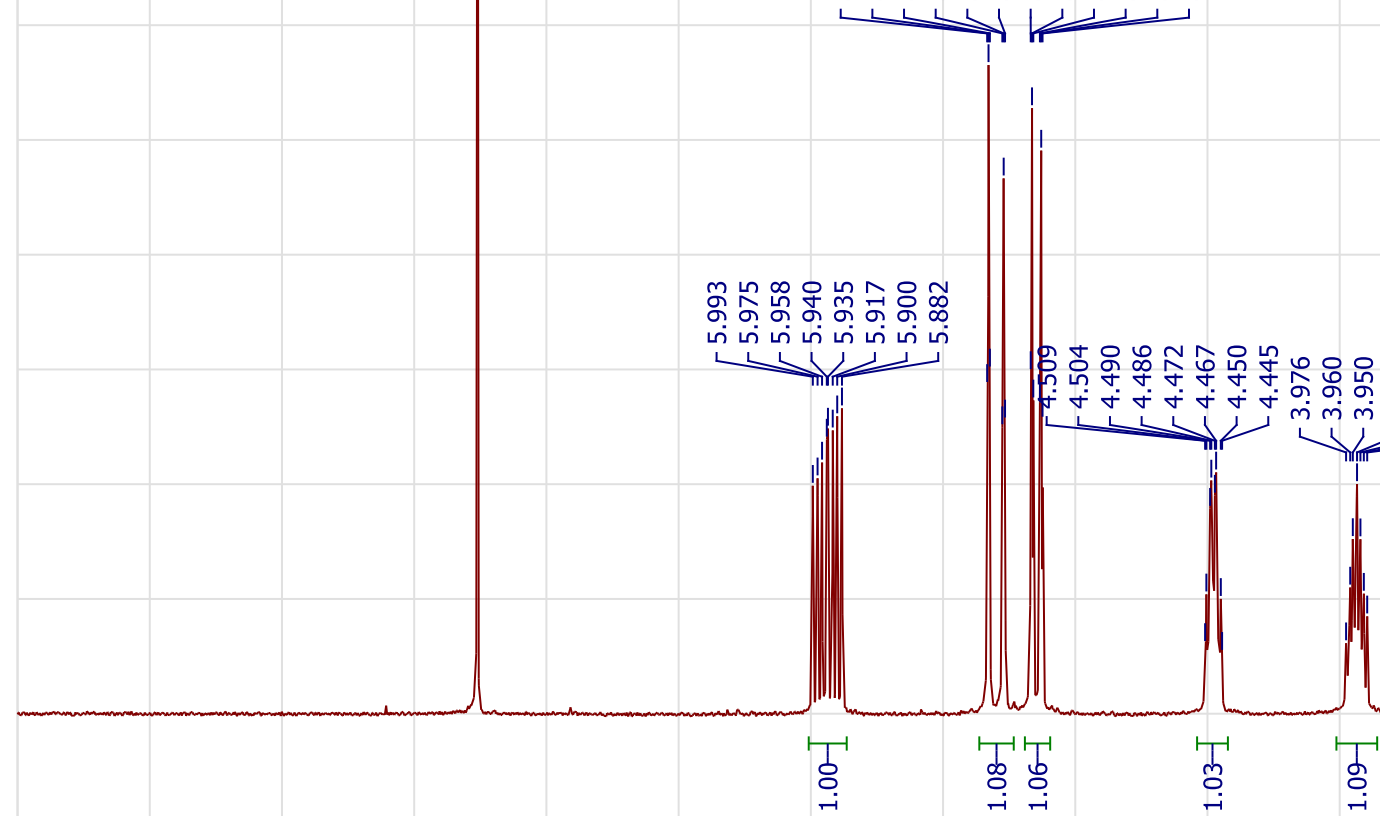

s
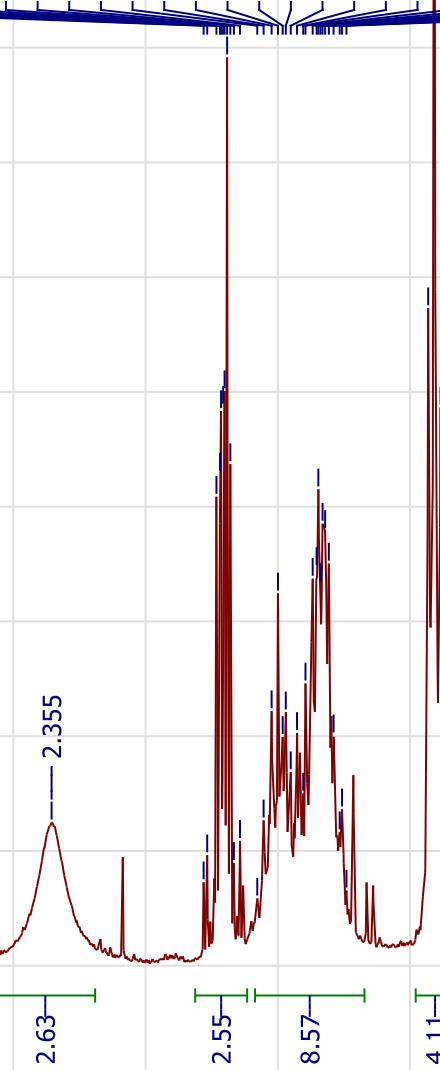


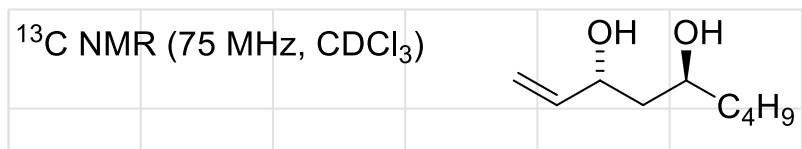

(士)-17

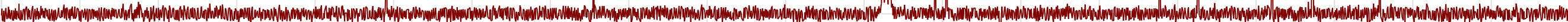

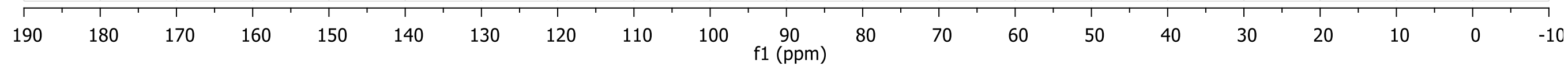


${ }^{1} \mathrm{H} \mathrm{NMR}\left(300 \mathrm{MHz}, \mathrm{CDCl}_{3}\right) \quad \mathrm{C}_{4} \mathrm{H}_{9 \prime \prime}$
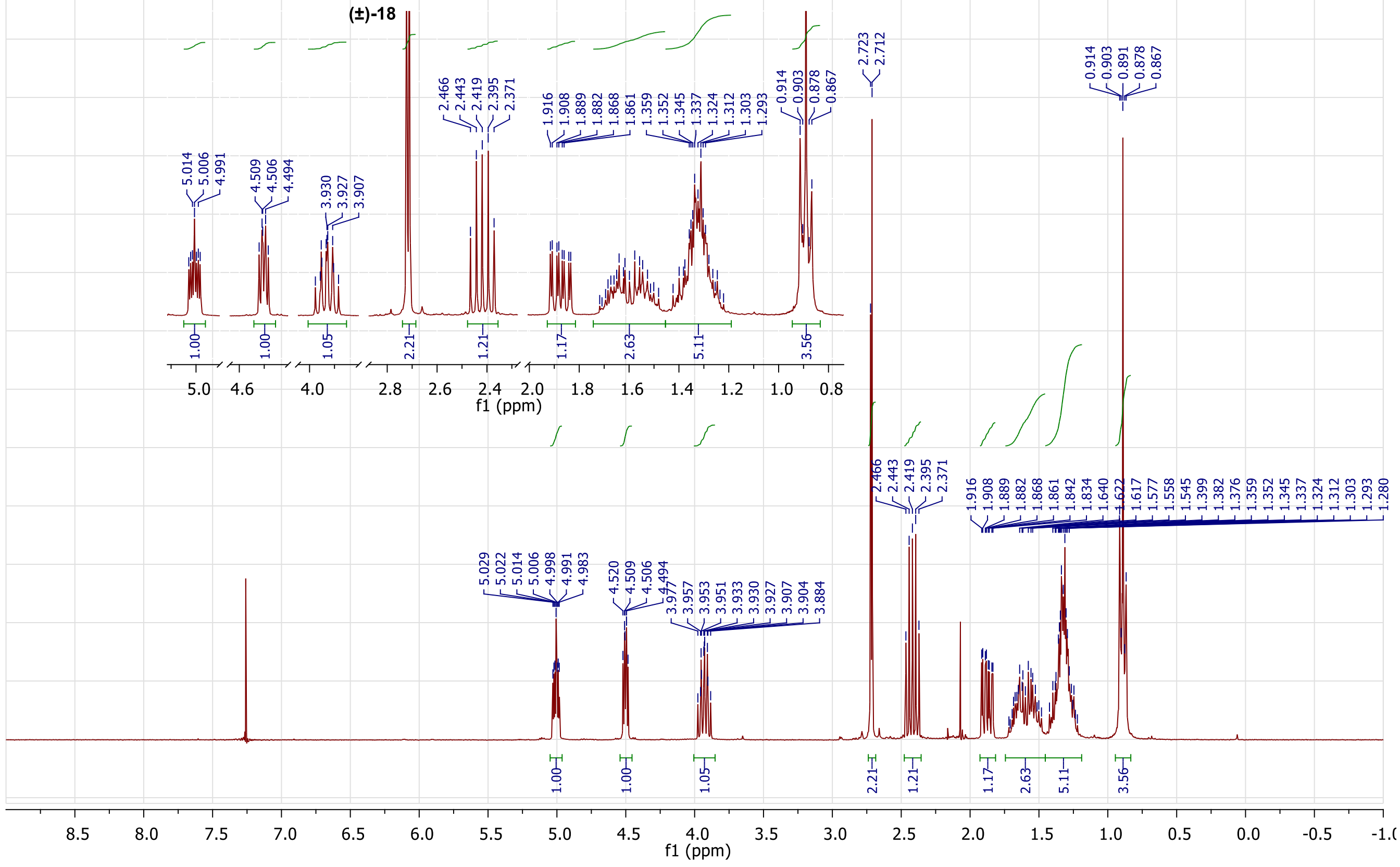


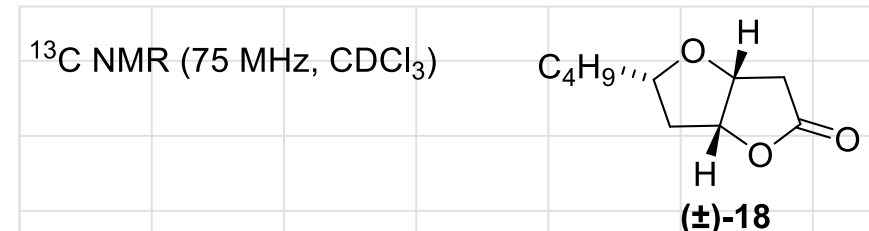

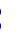

ก

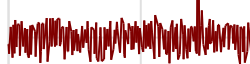

(士)-18 


\section{${ }^{1} \mathrm{H} \mathrm{NMR}\left(300 \mathrm{MHz}, \mathrm{CDCl}_{3}\right)$ \\ $\overbrace{(+-19}^{\mathrm{OH}}$}

$( \pm)-19$

\section{$\beta / 1$}

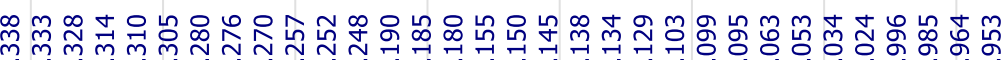

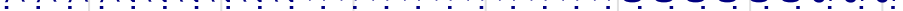

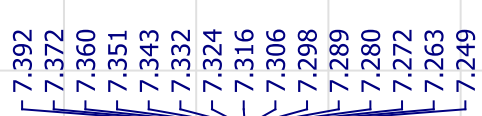

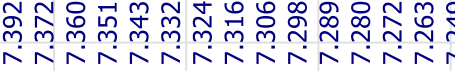
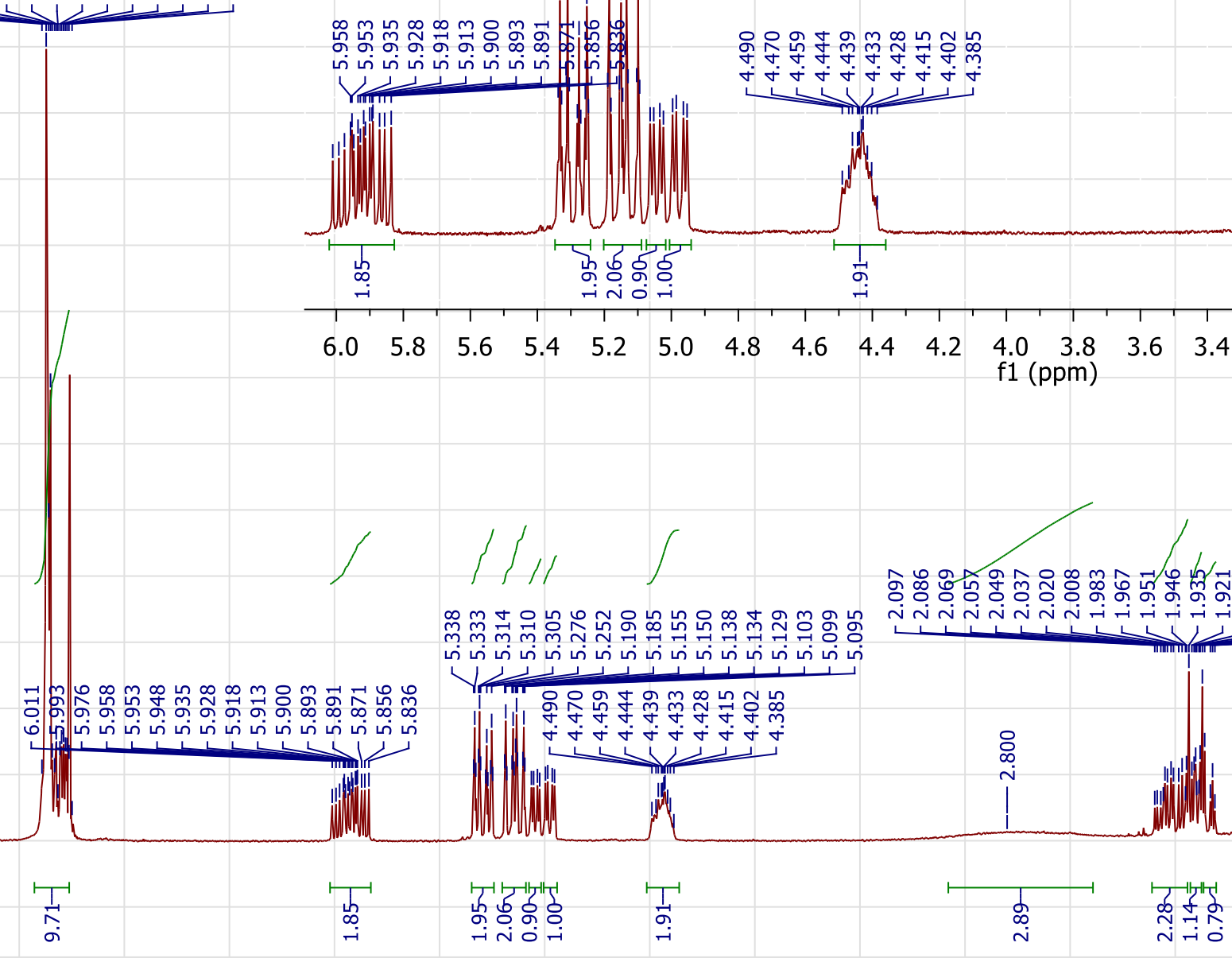

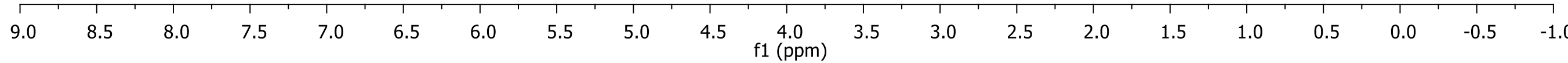




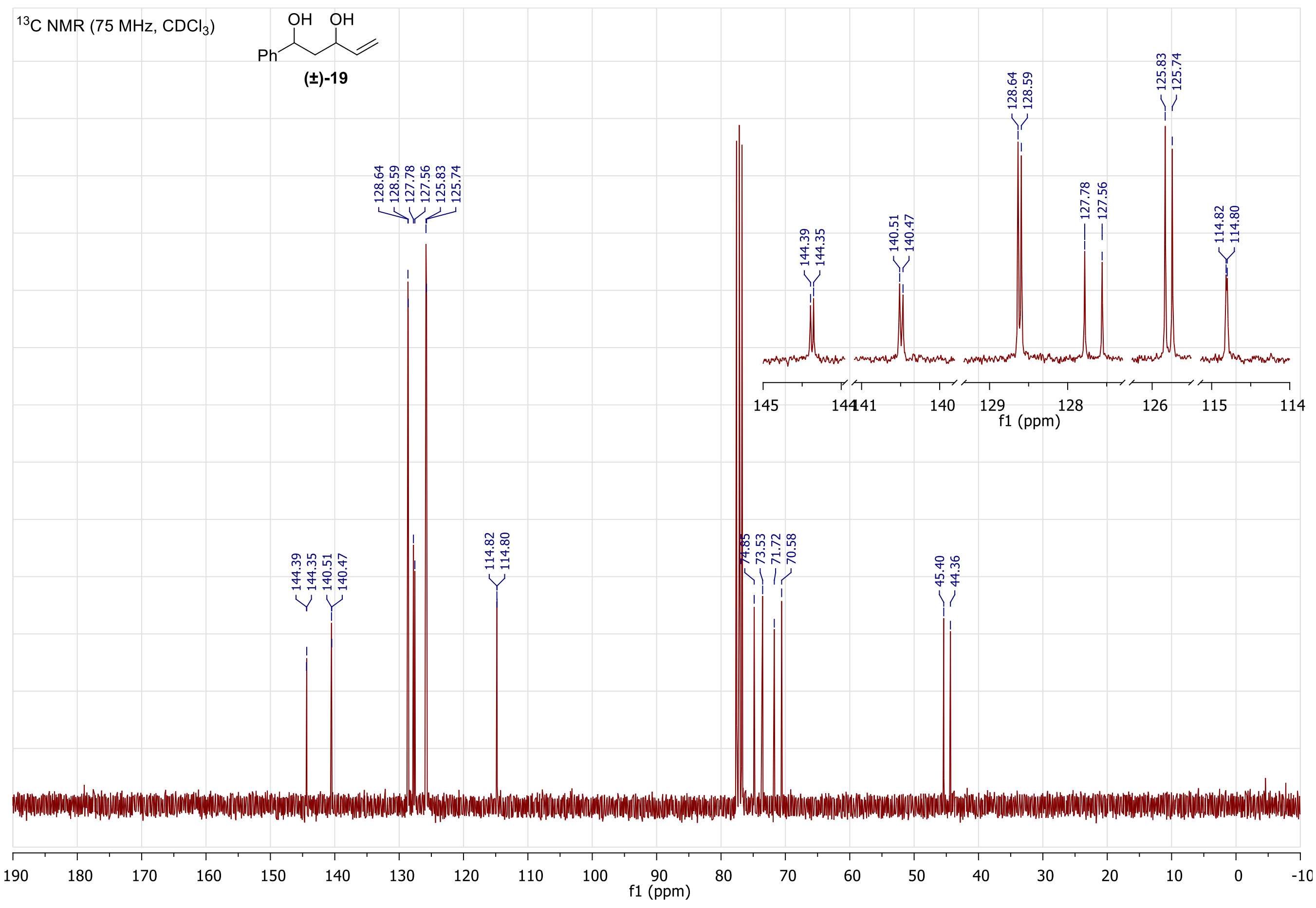


${ }^{1} \mathrm{H} \mathrm{NMR}\left(300 \mathrm{MHz}, \mathrm{CDCl}_{3}\right) \quad \mathrm{Ph}_{1 .}$

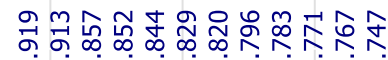

(士)-endo-20
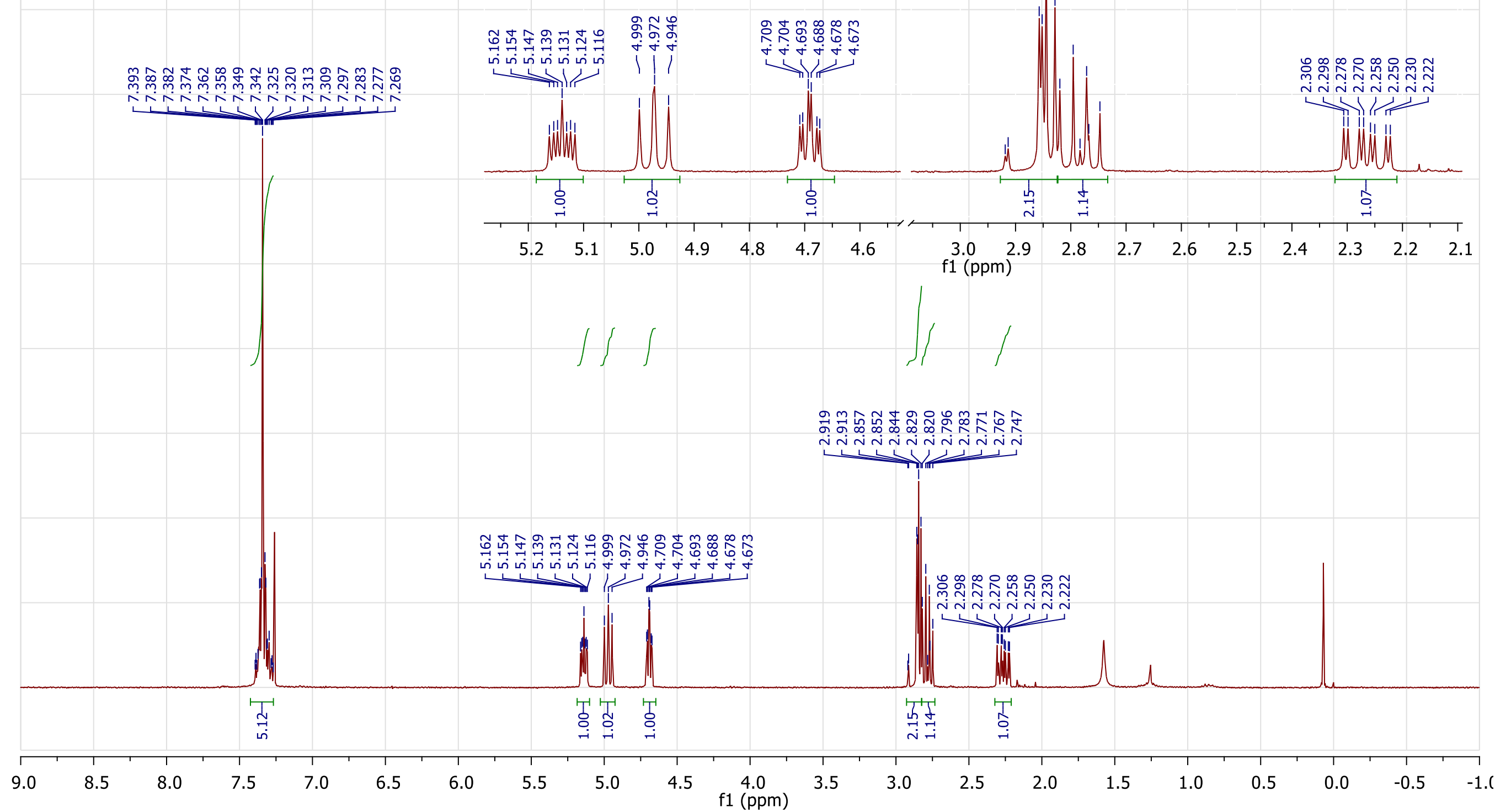


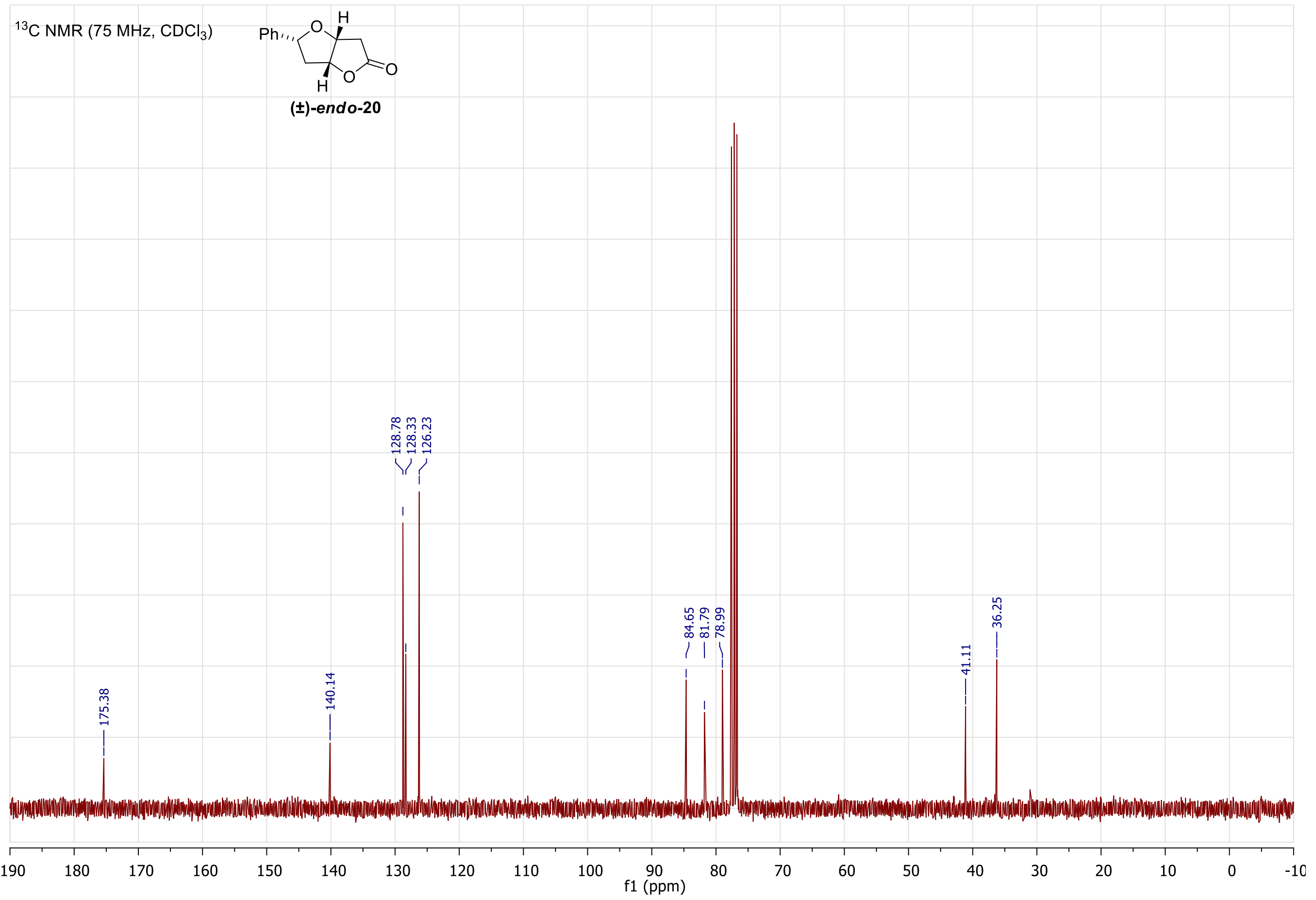

S69 
${ }^{1} \mathrm{HNMR}\left(300 \mathrm{MHz}, \mathrm{CDCl}_{3}\right)$
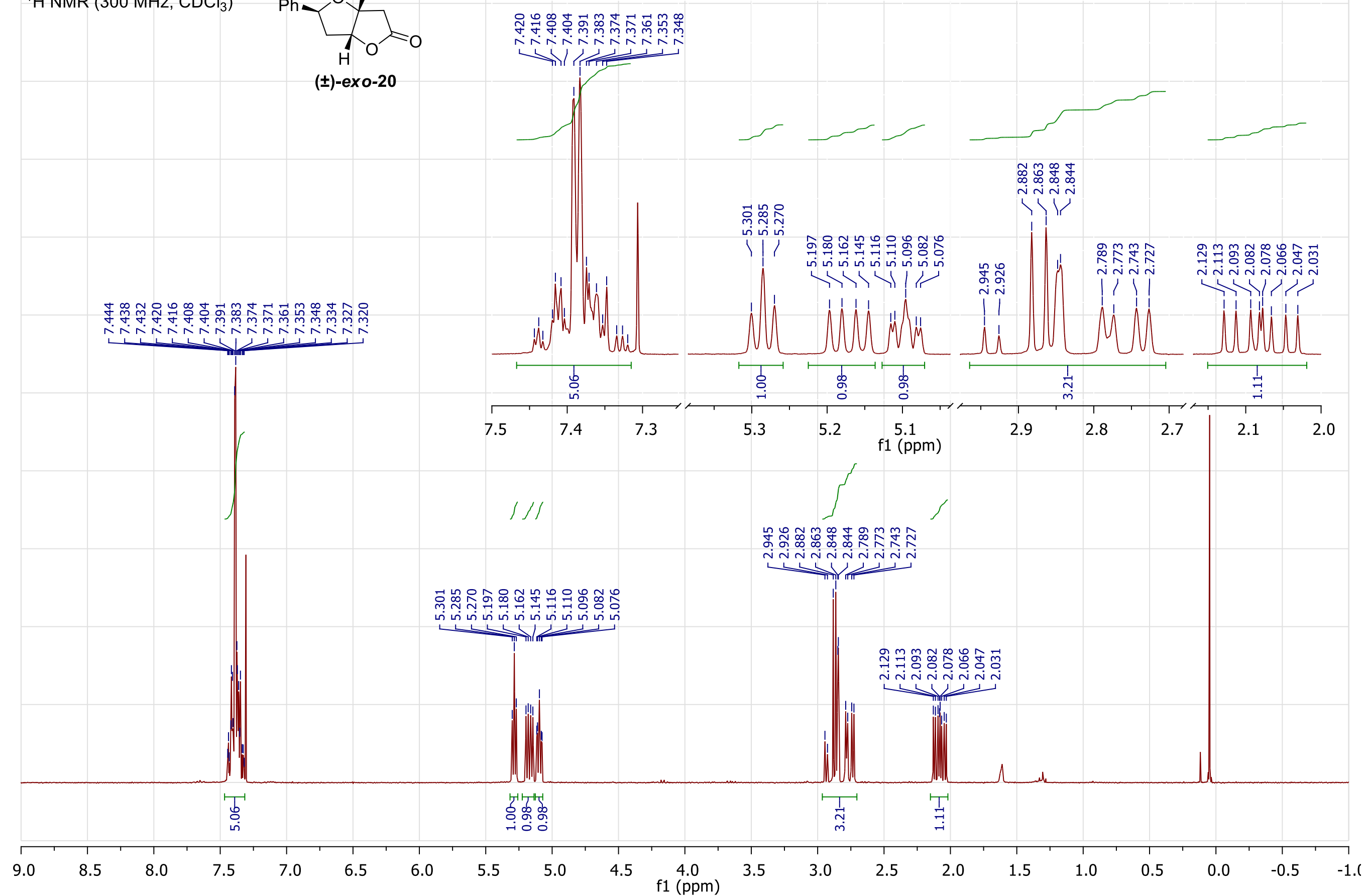

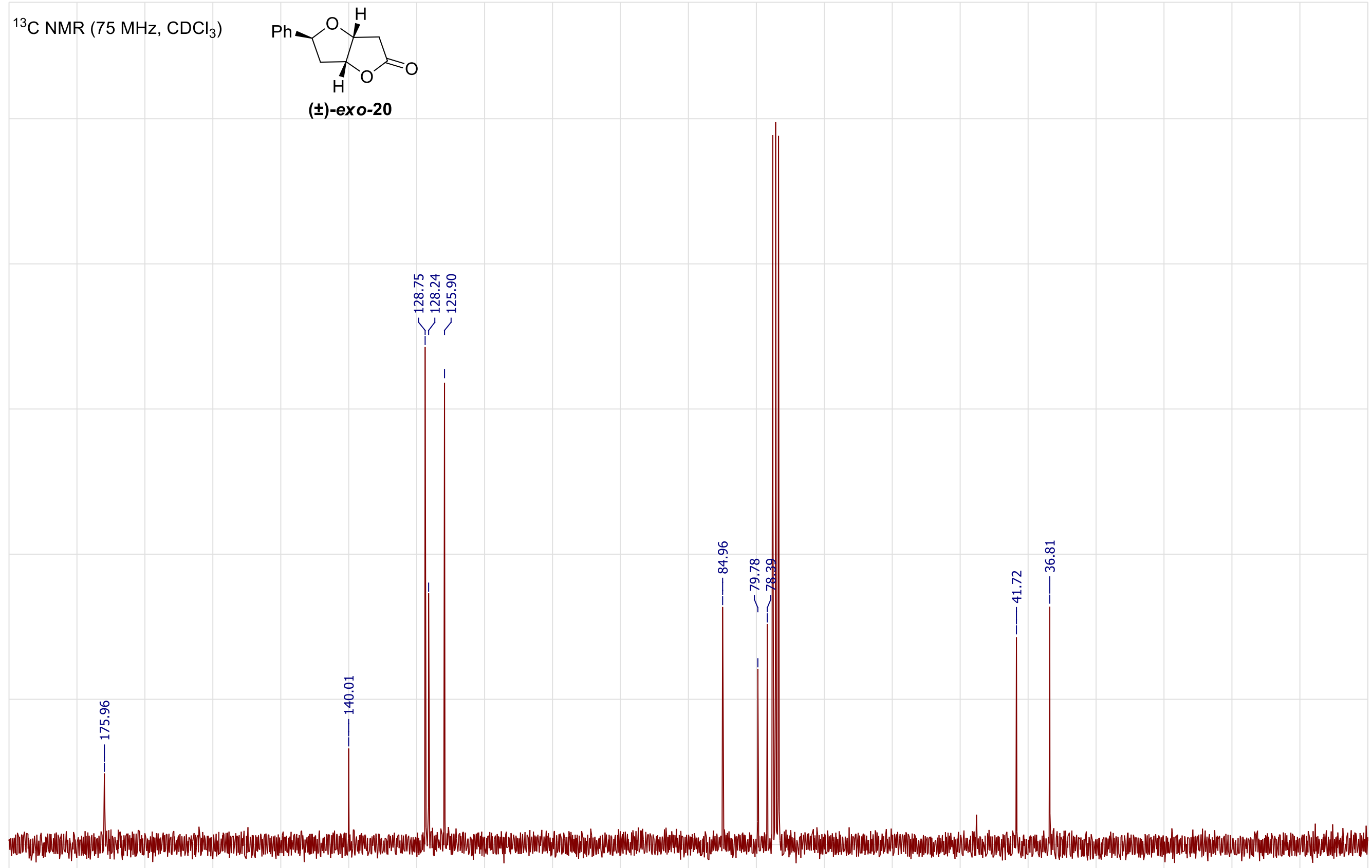

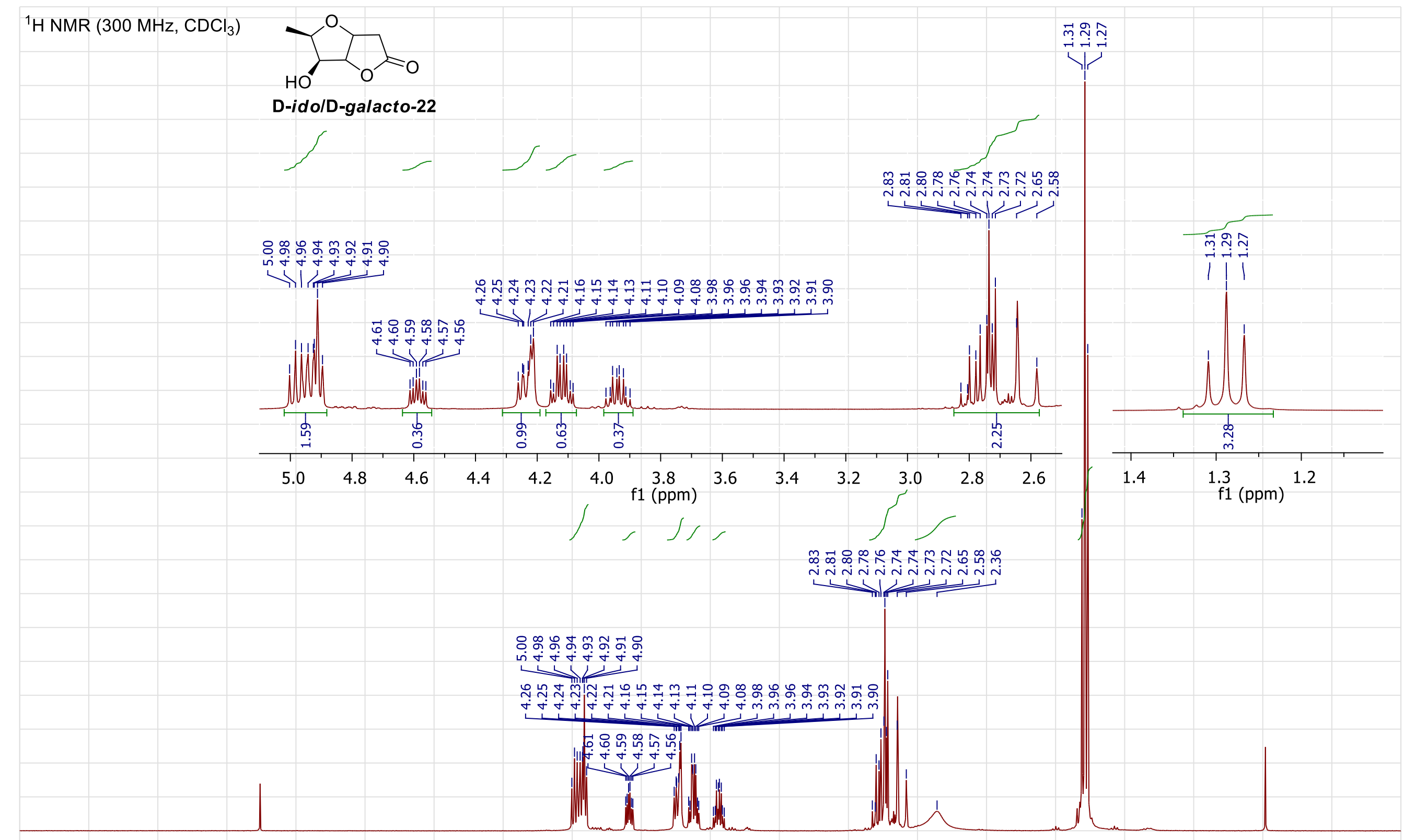

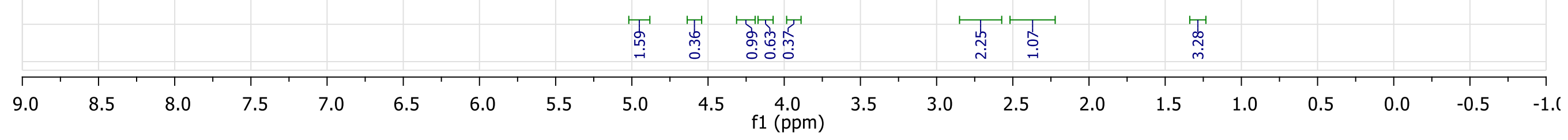



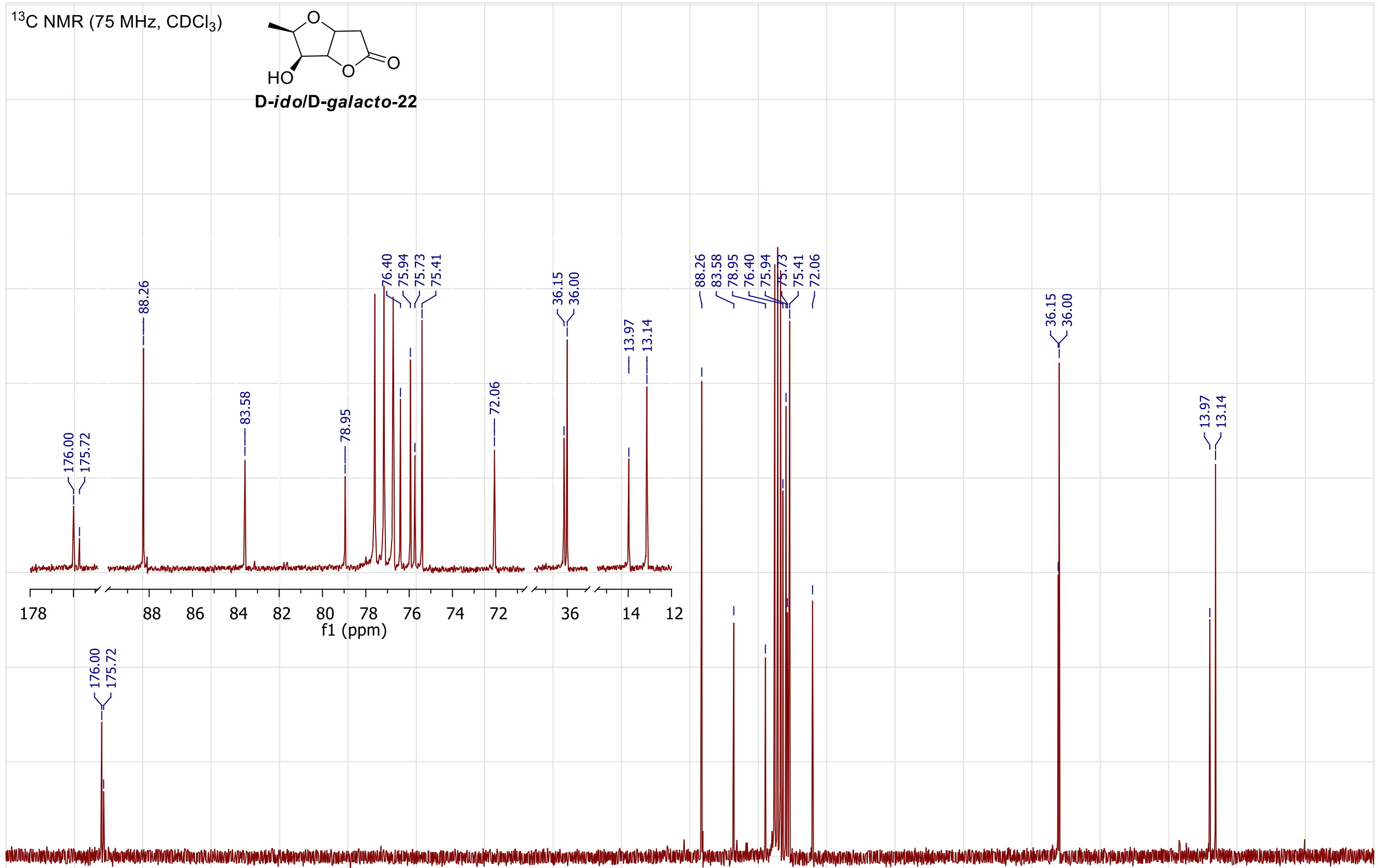

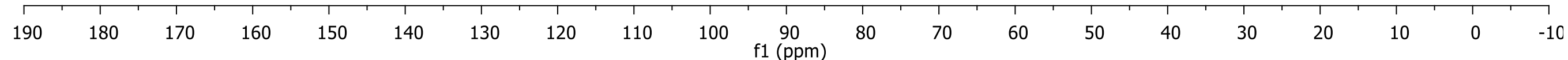




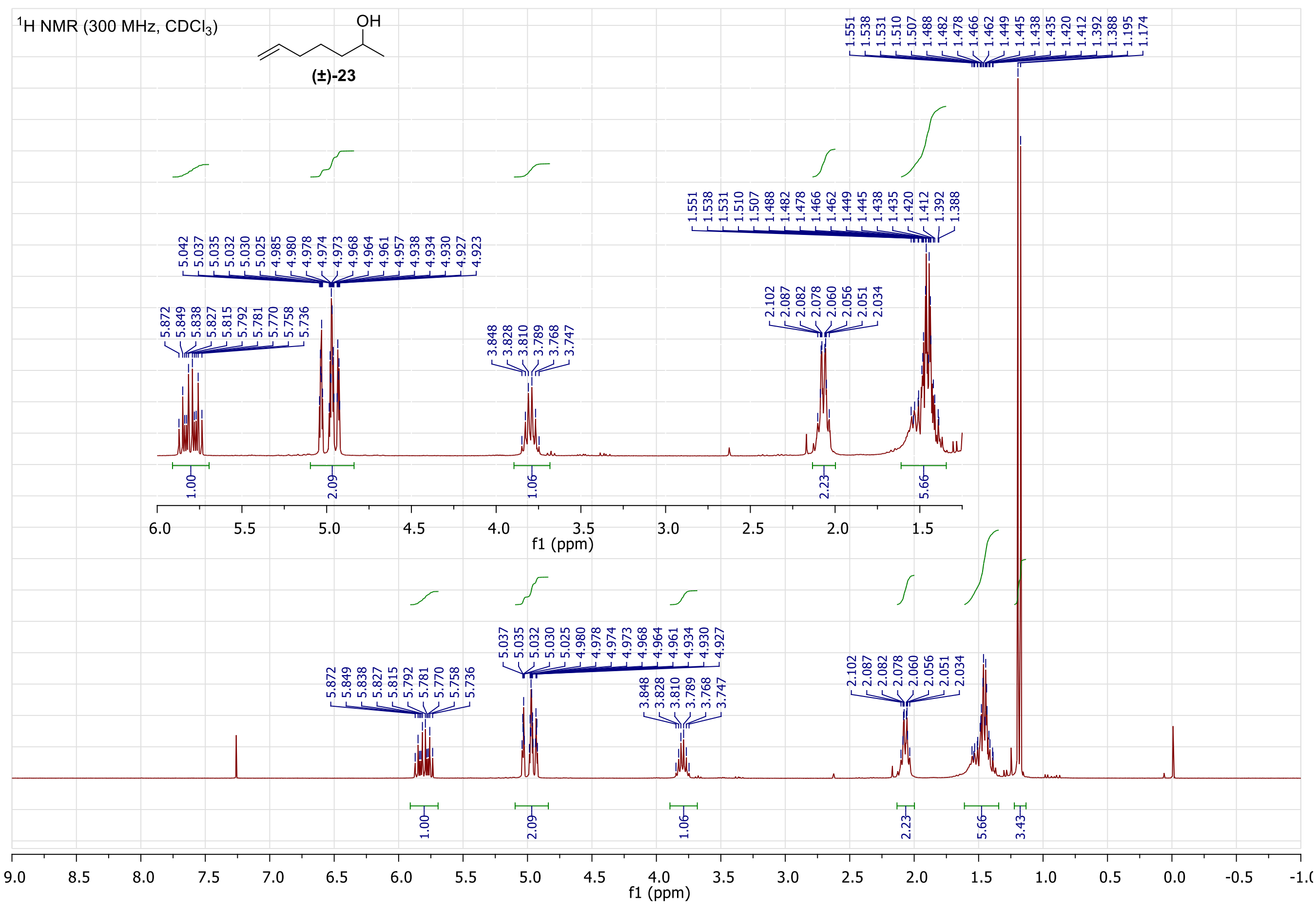




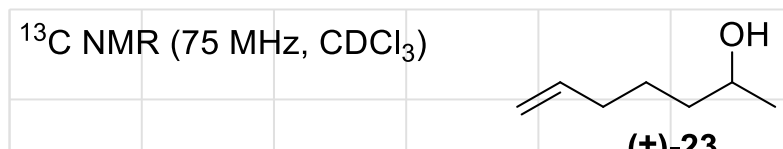

(士)-23

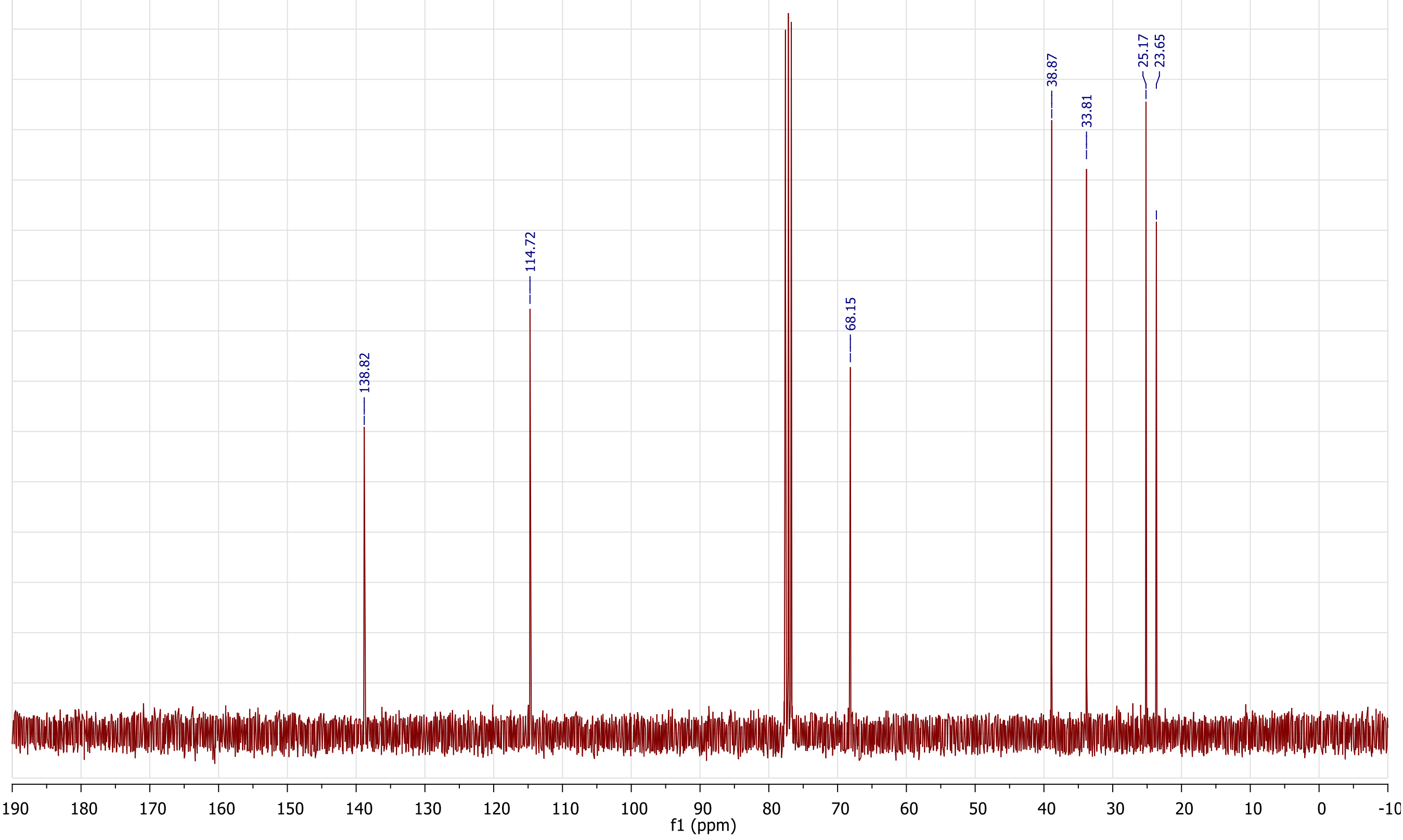




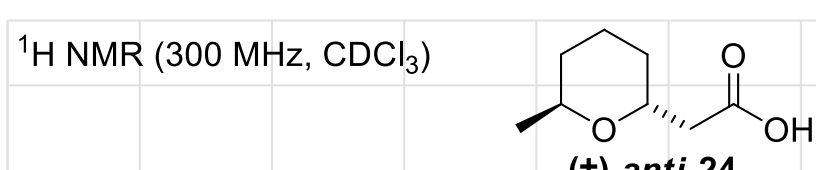

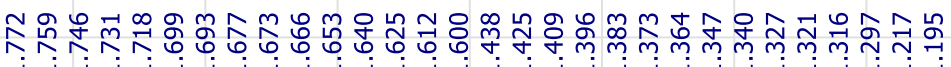

(士)-anti-24

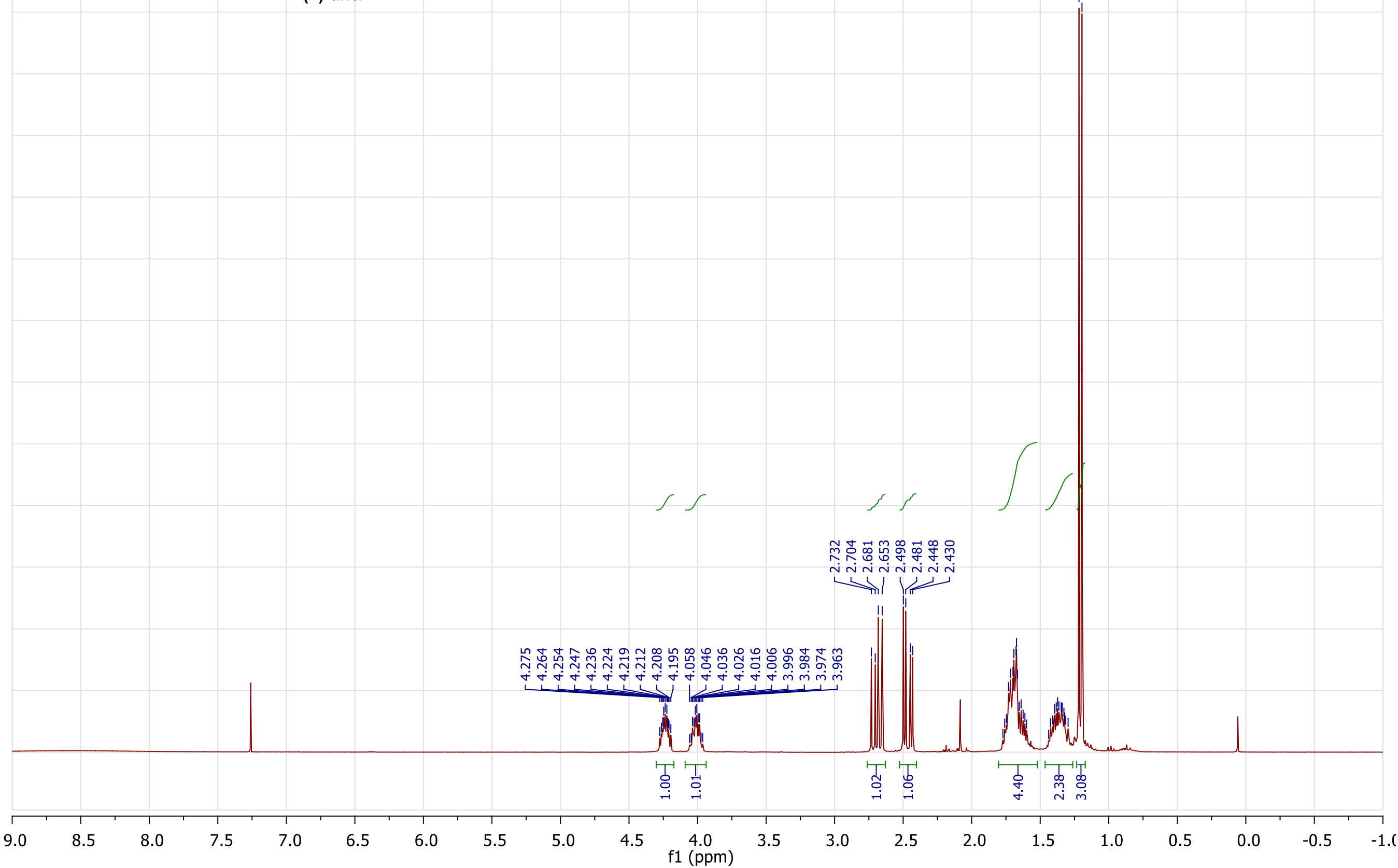




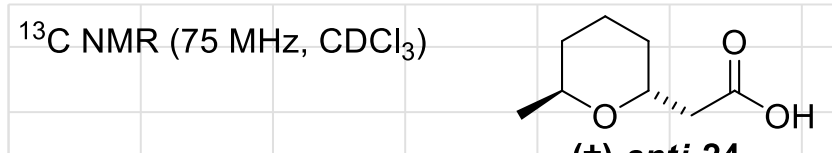

(士)-anti-24

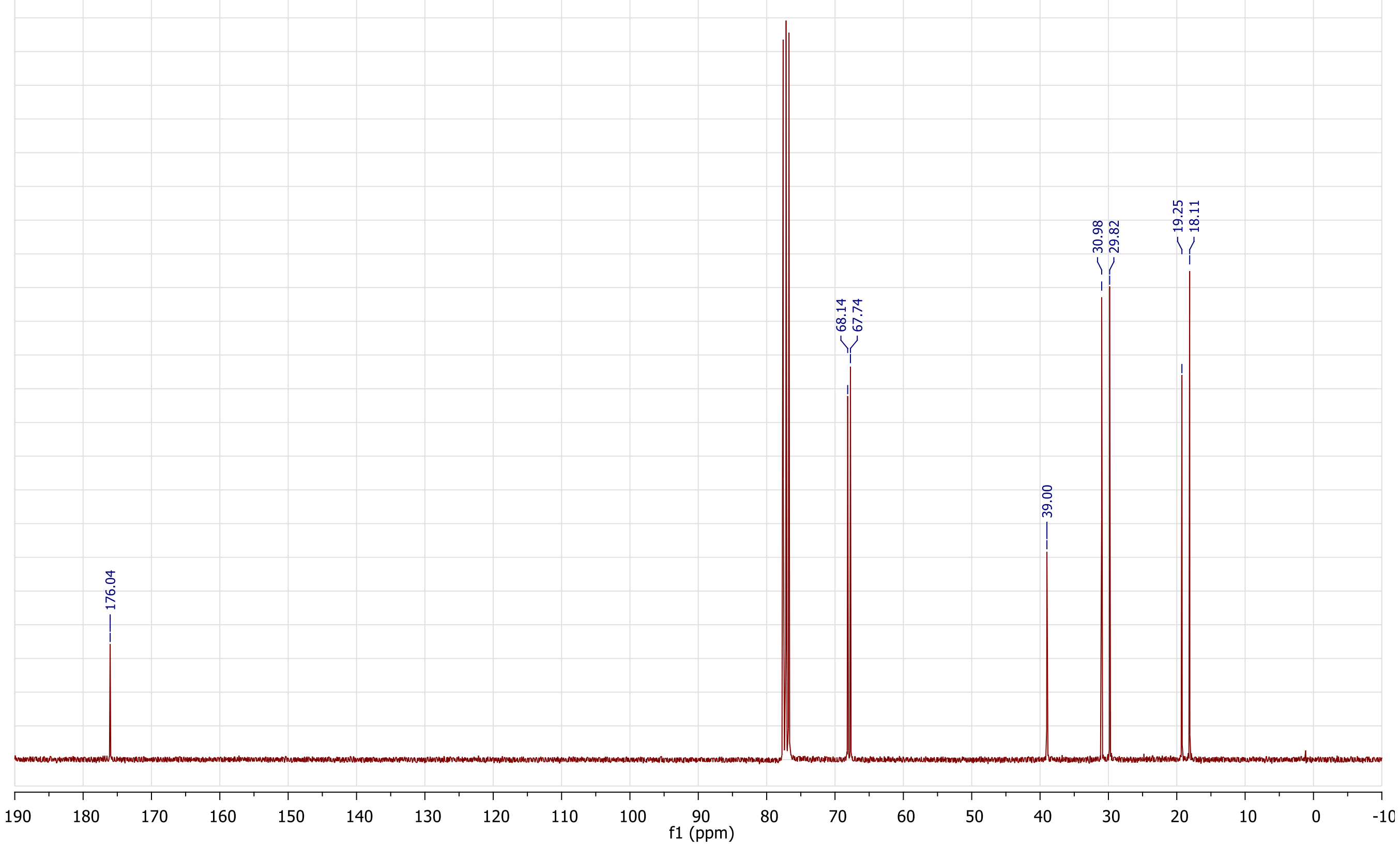




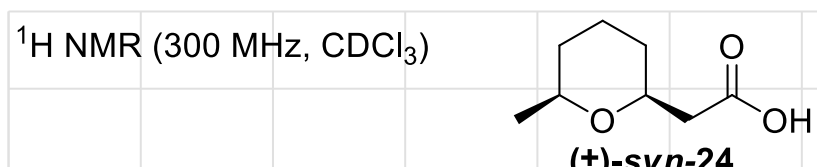

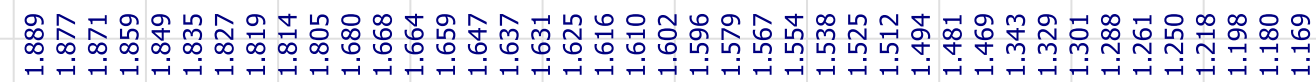

(士)-syn-24
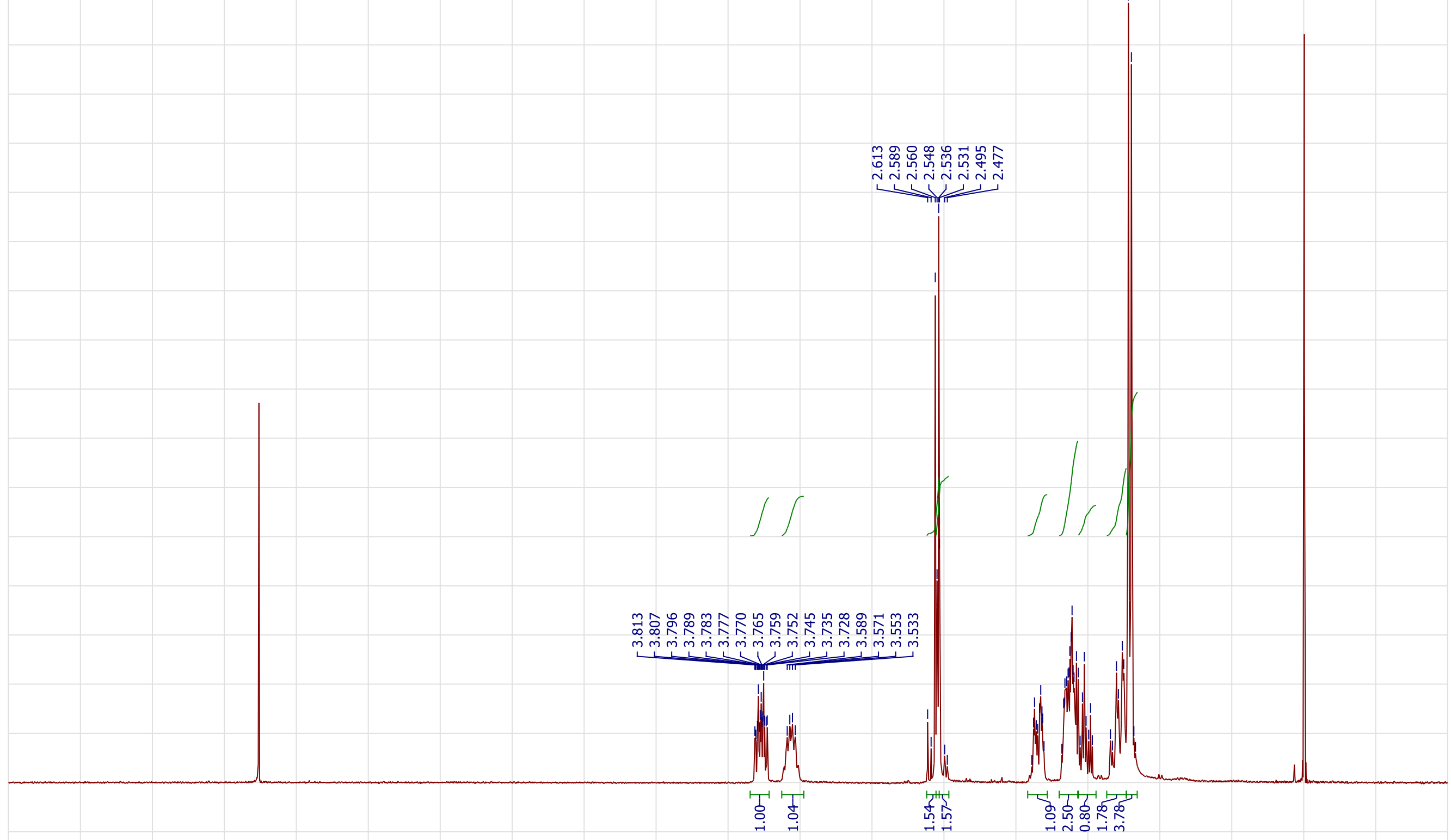


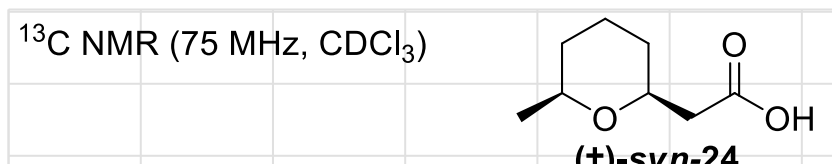

(士)-syn-24

点

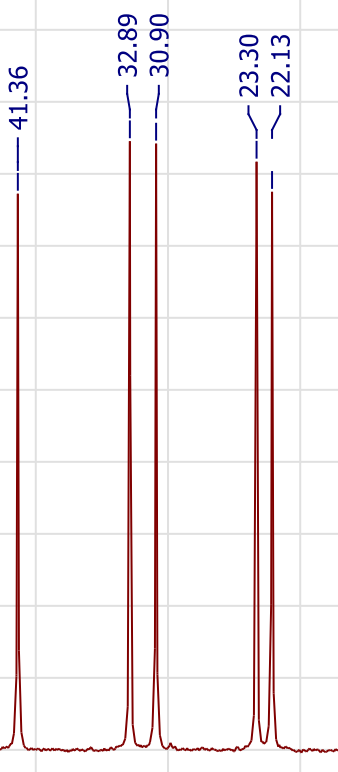

กิบ

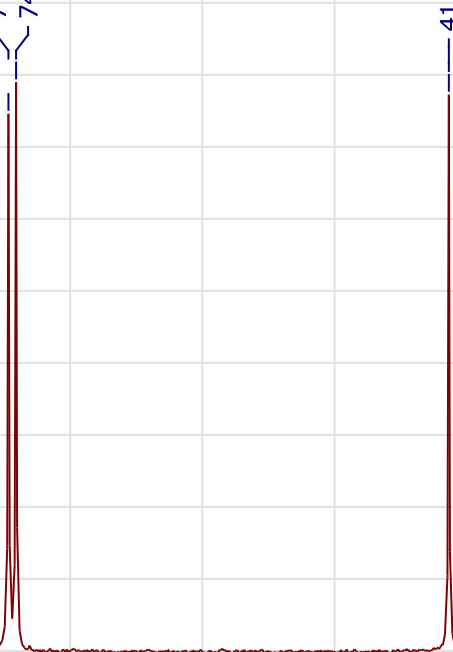

d 


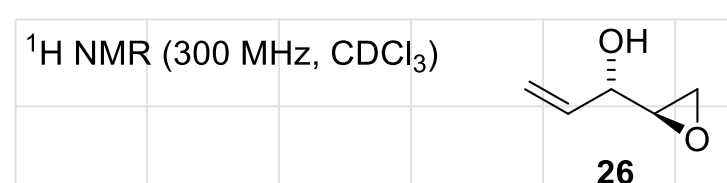

26

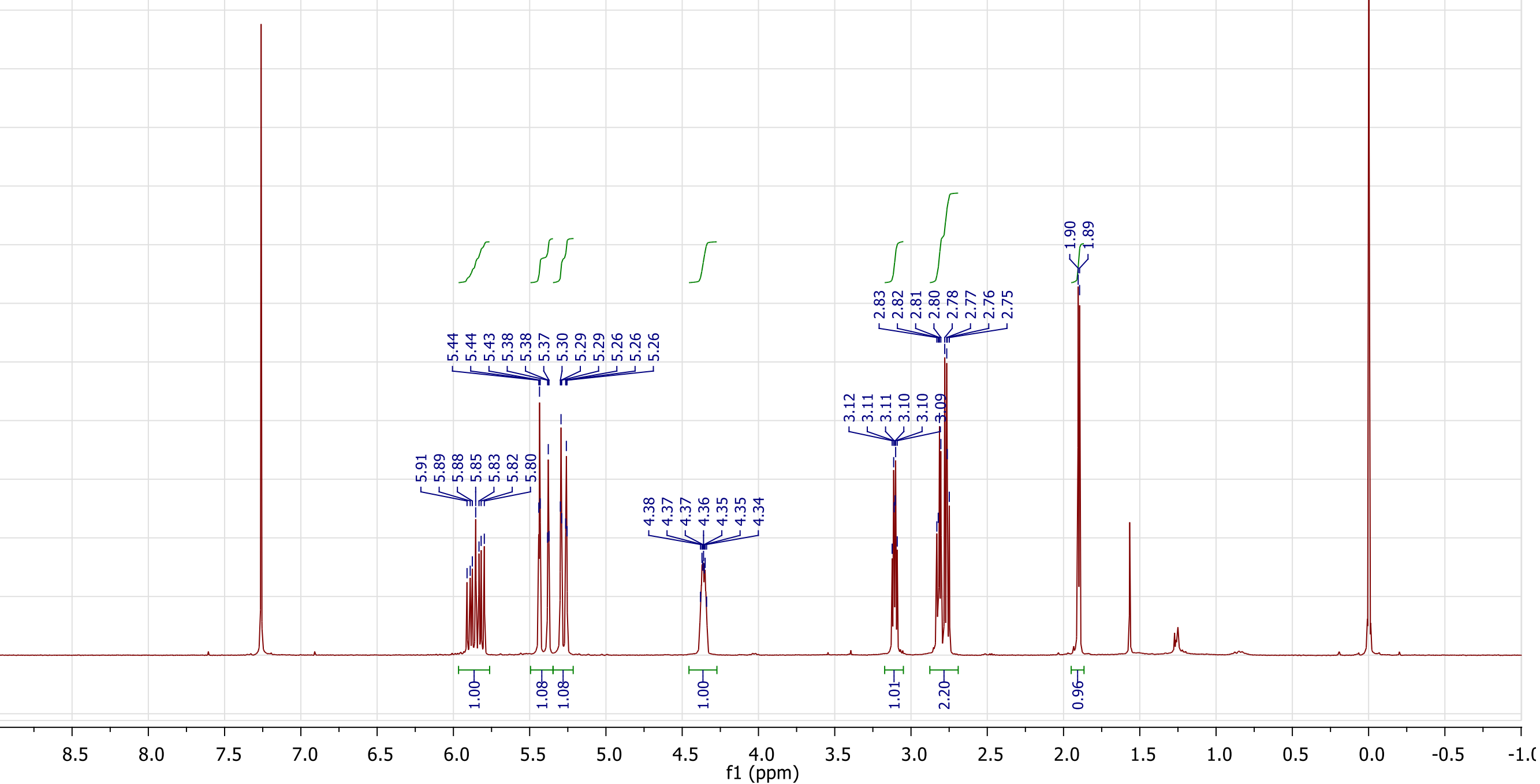




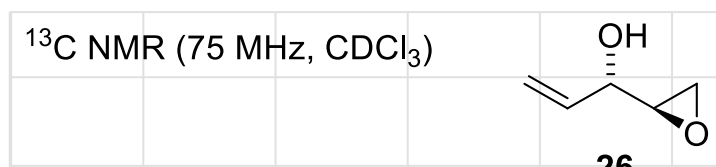

26

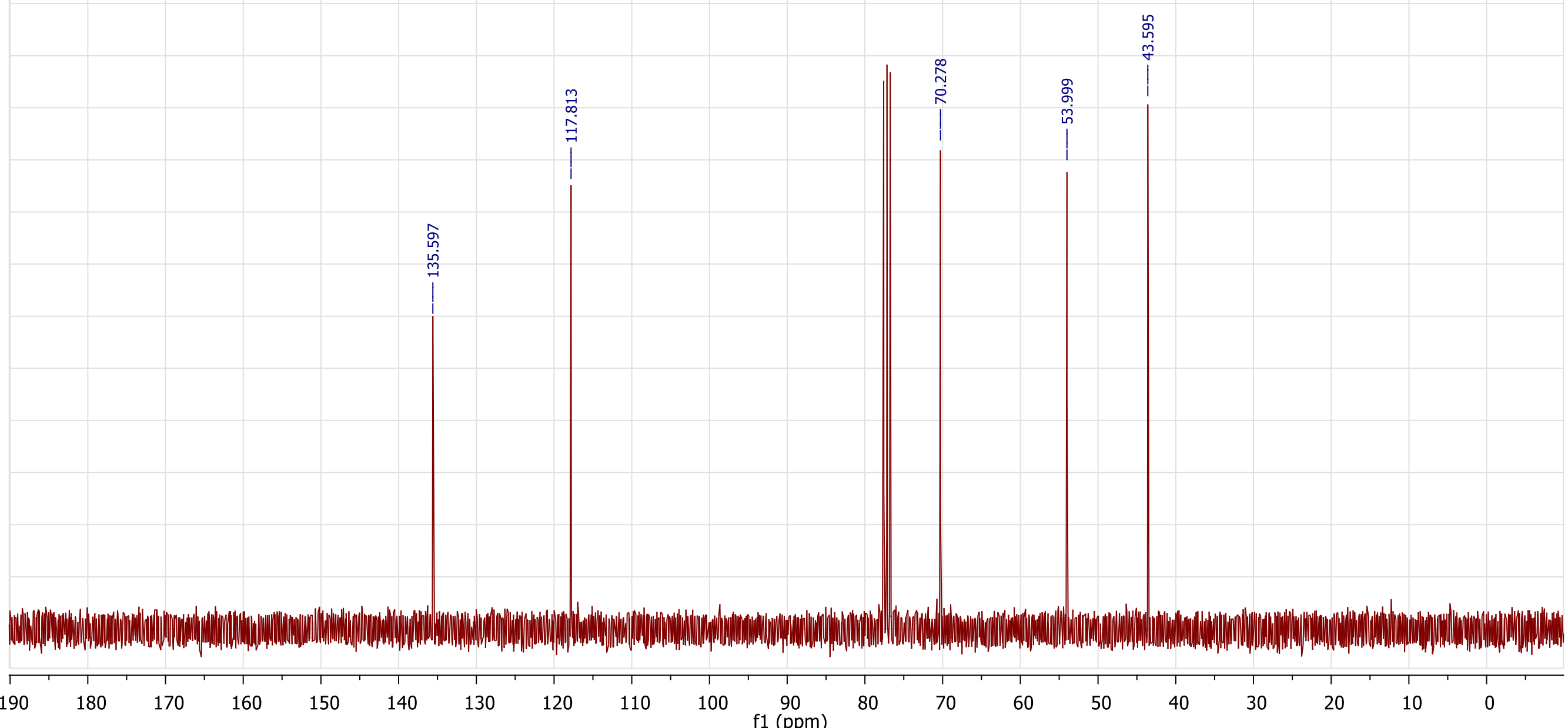




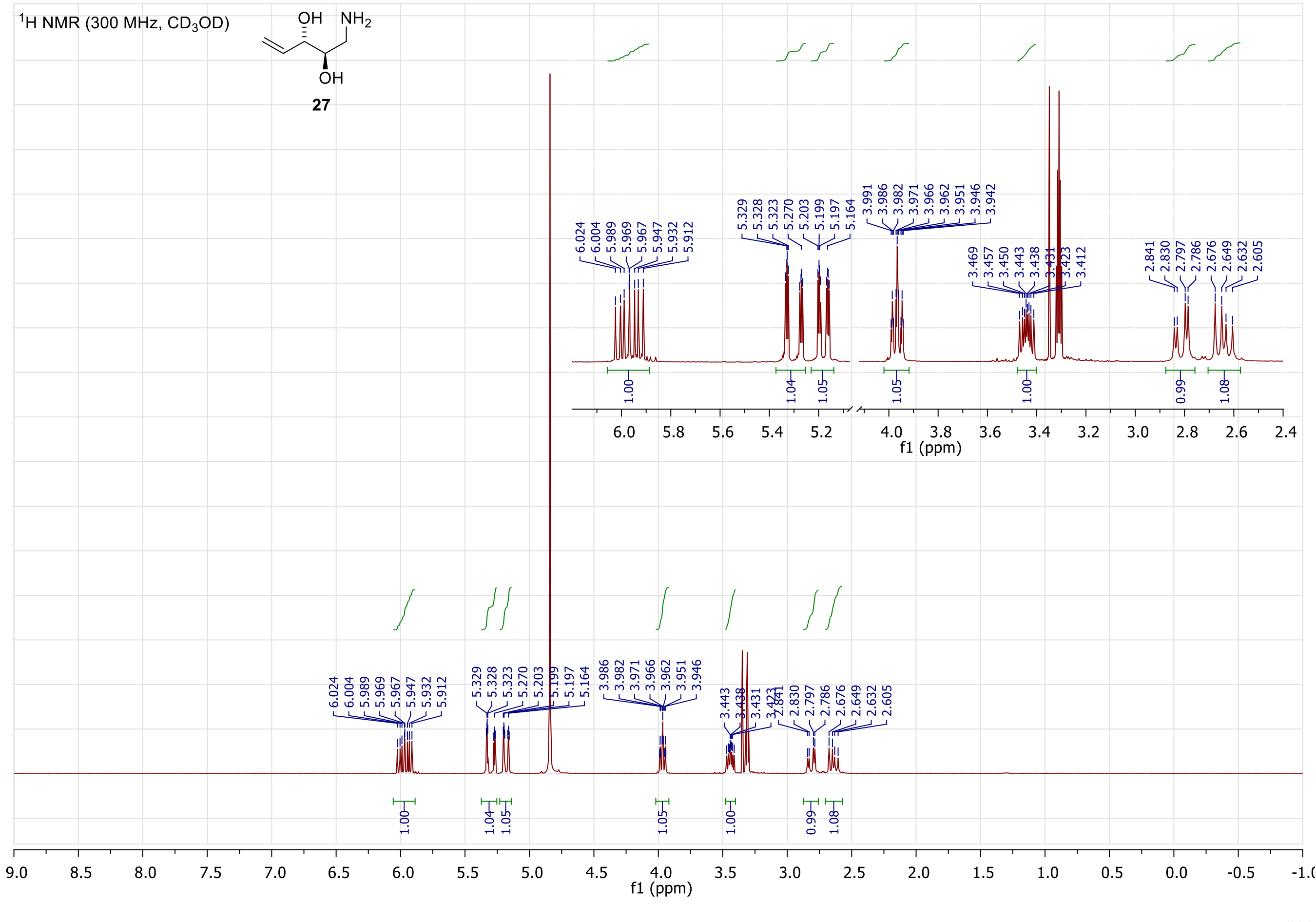




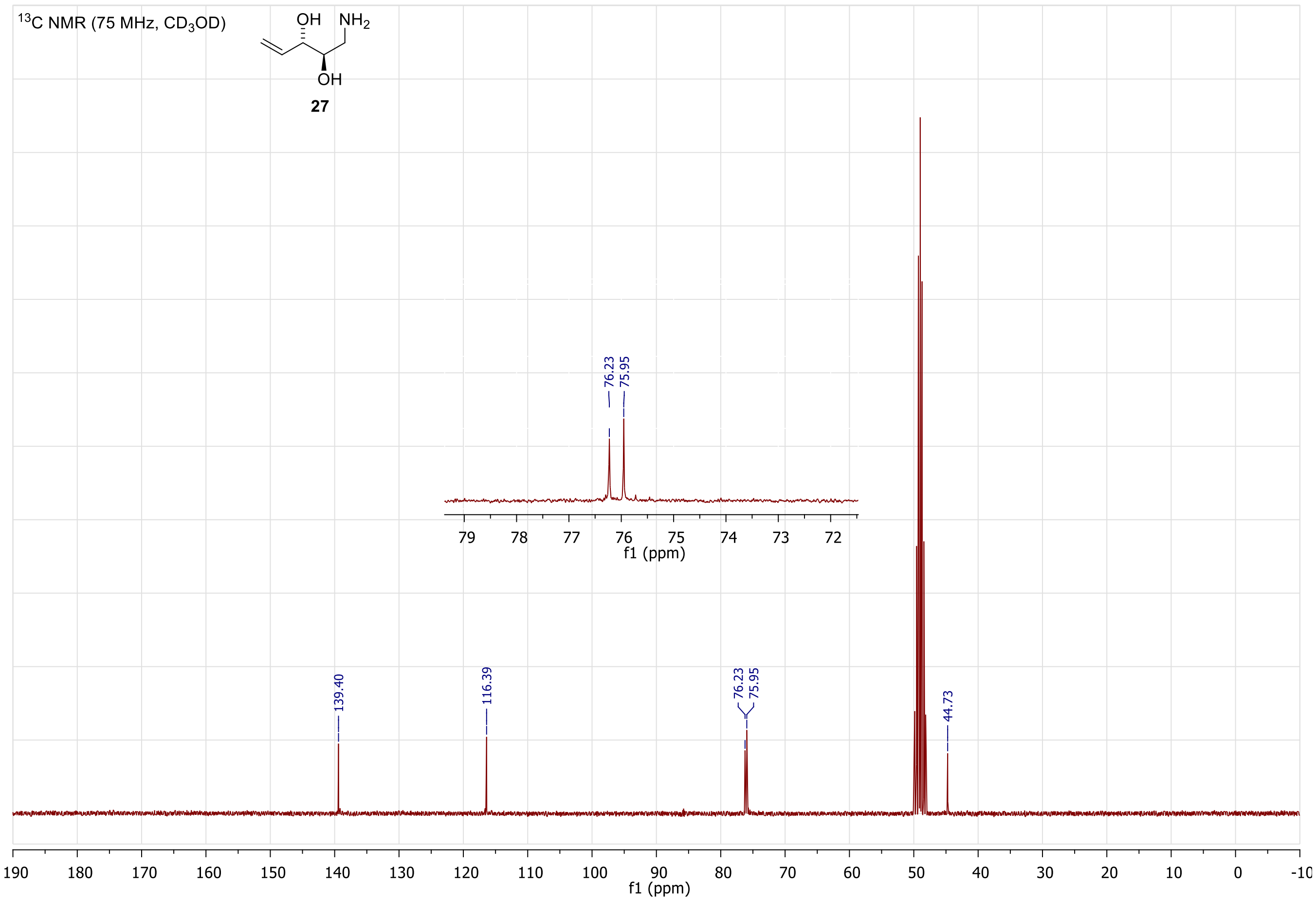




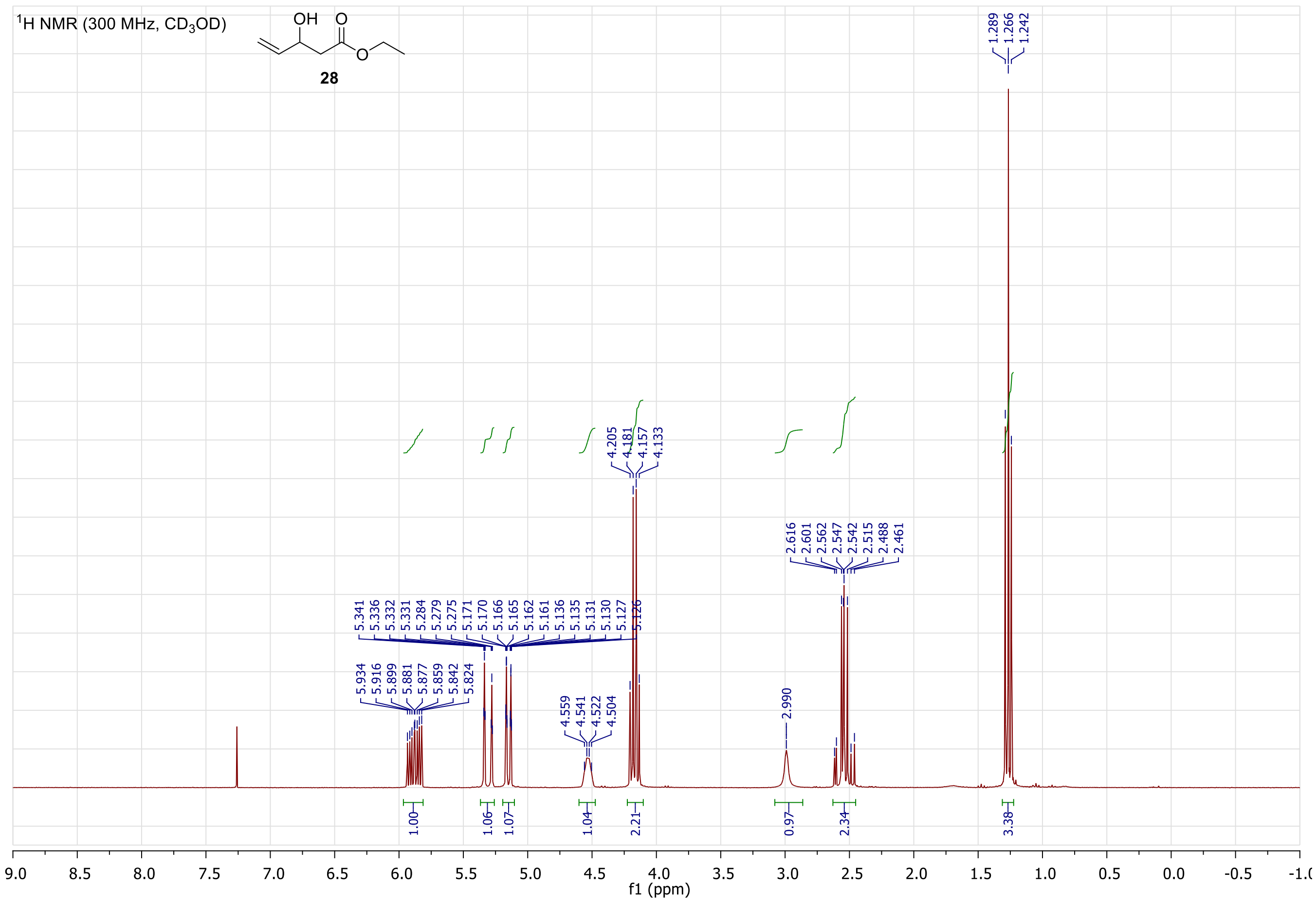




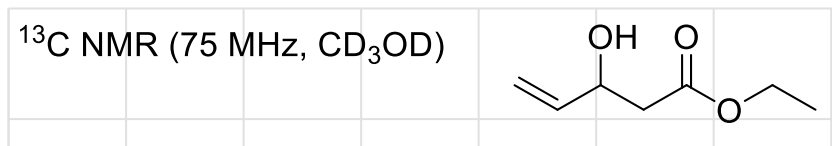

28

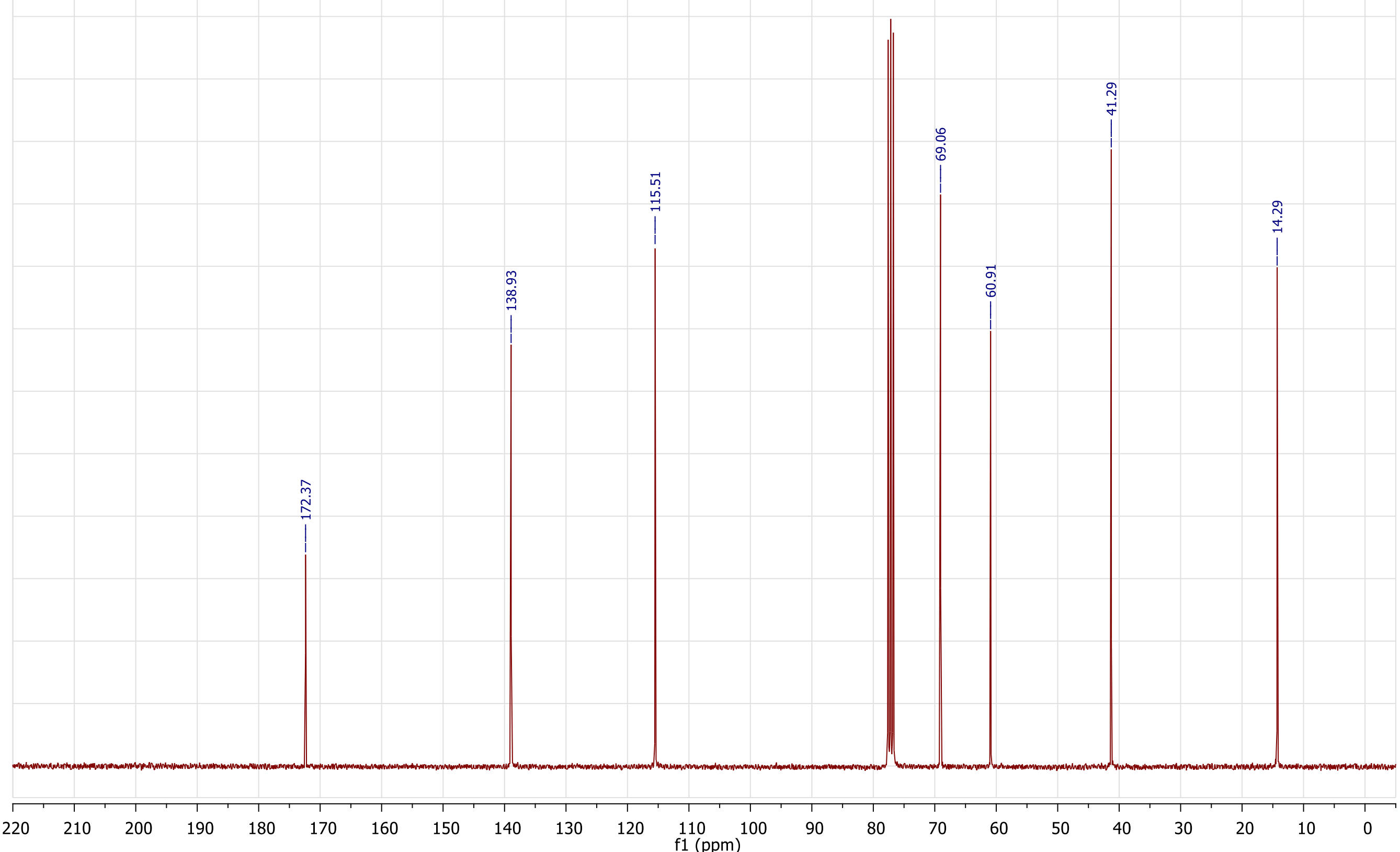




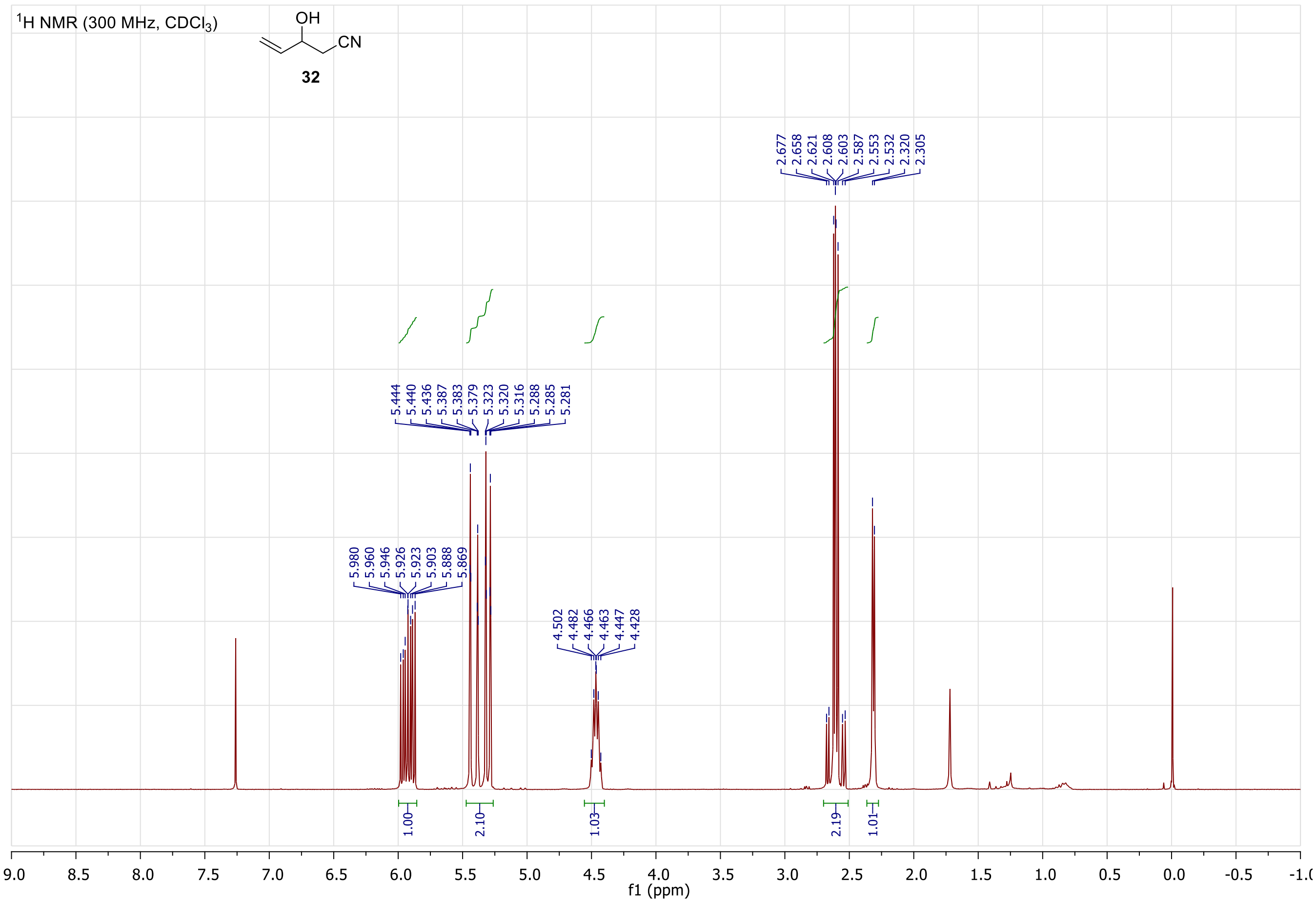




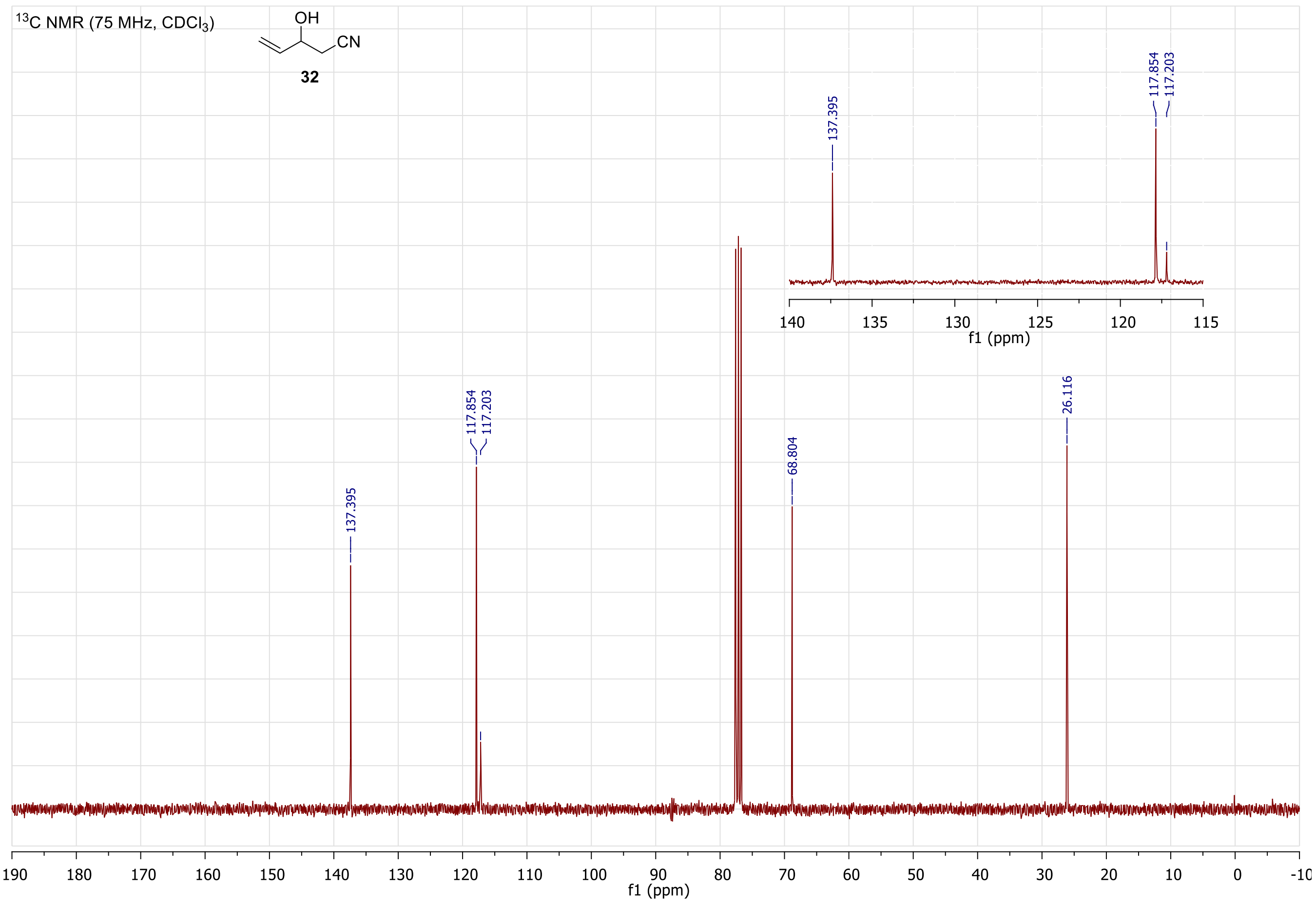




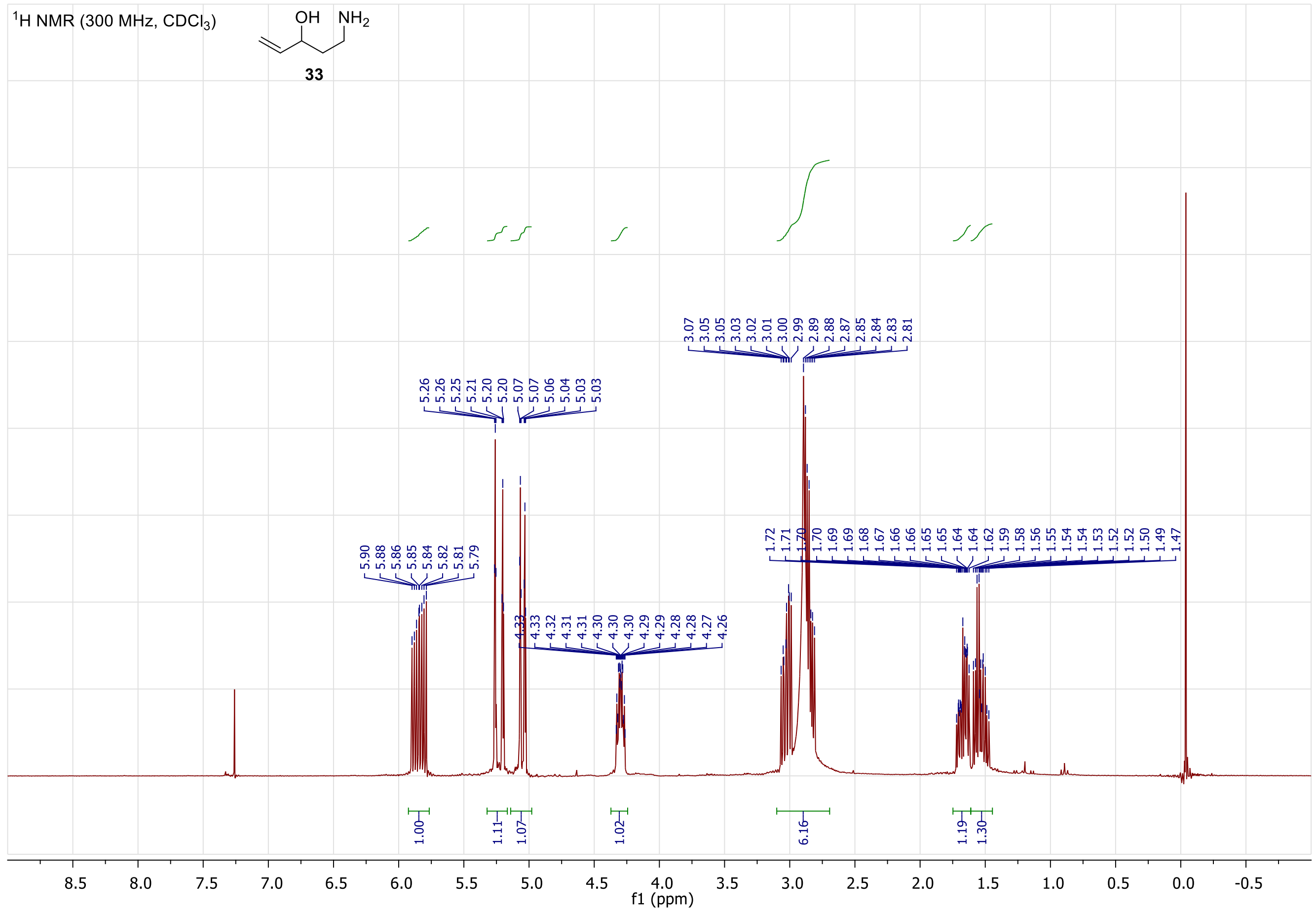




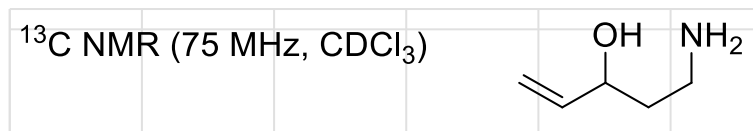

33

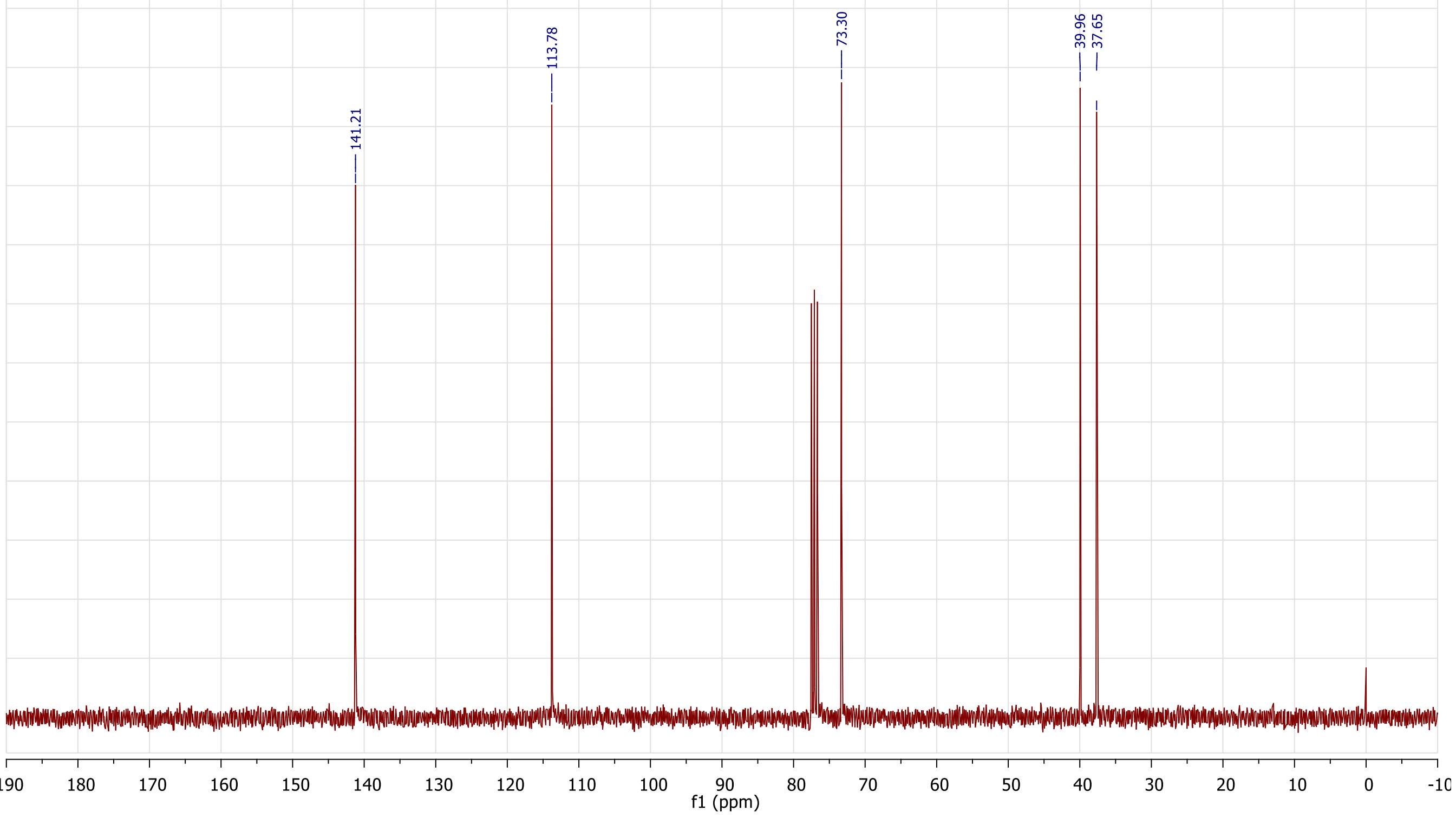



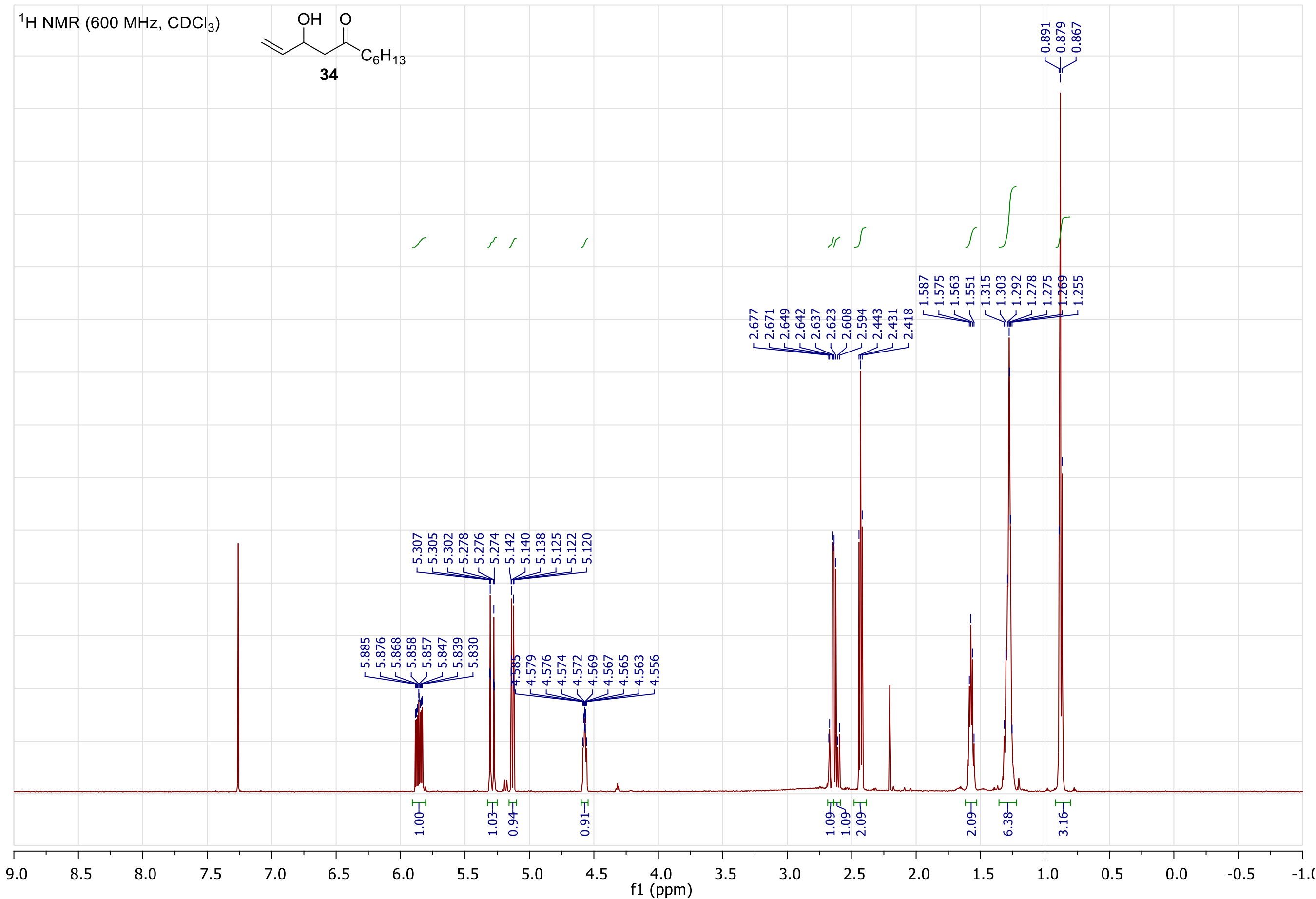

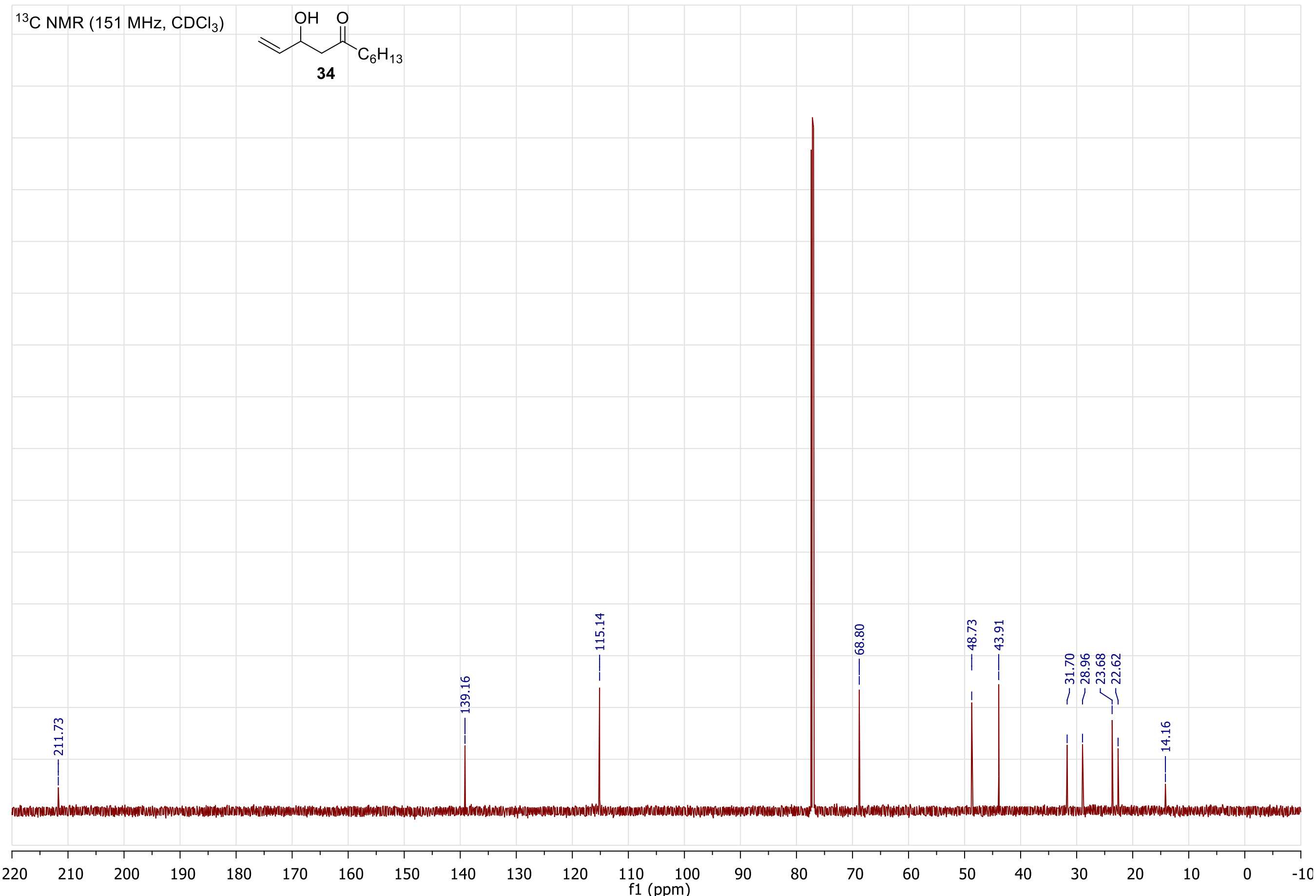

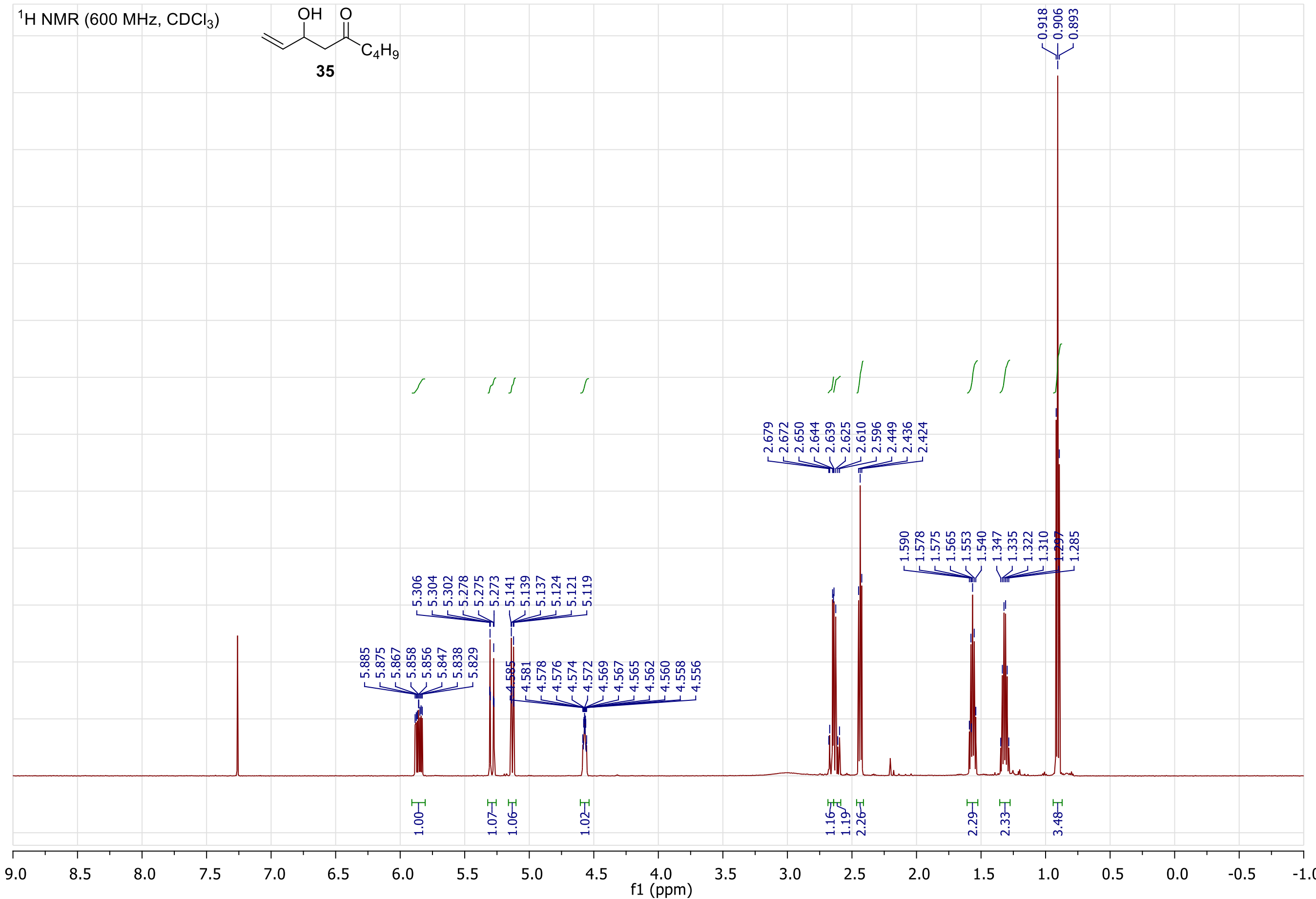

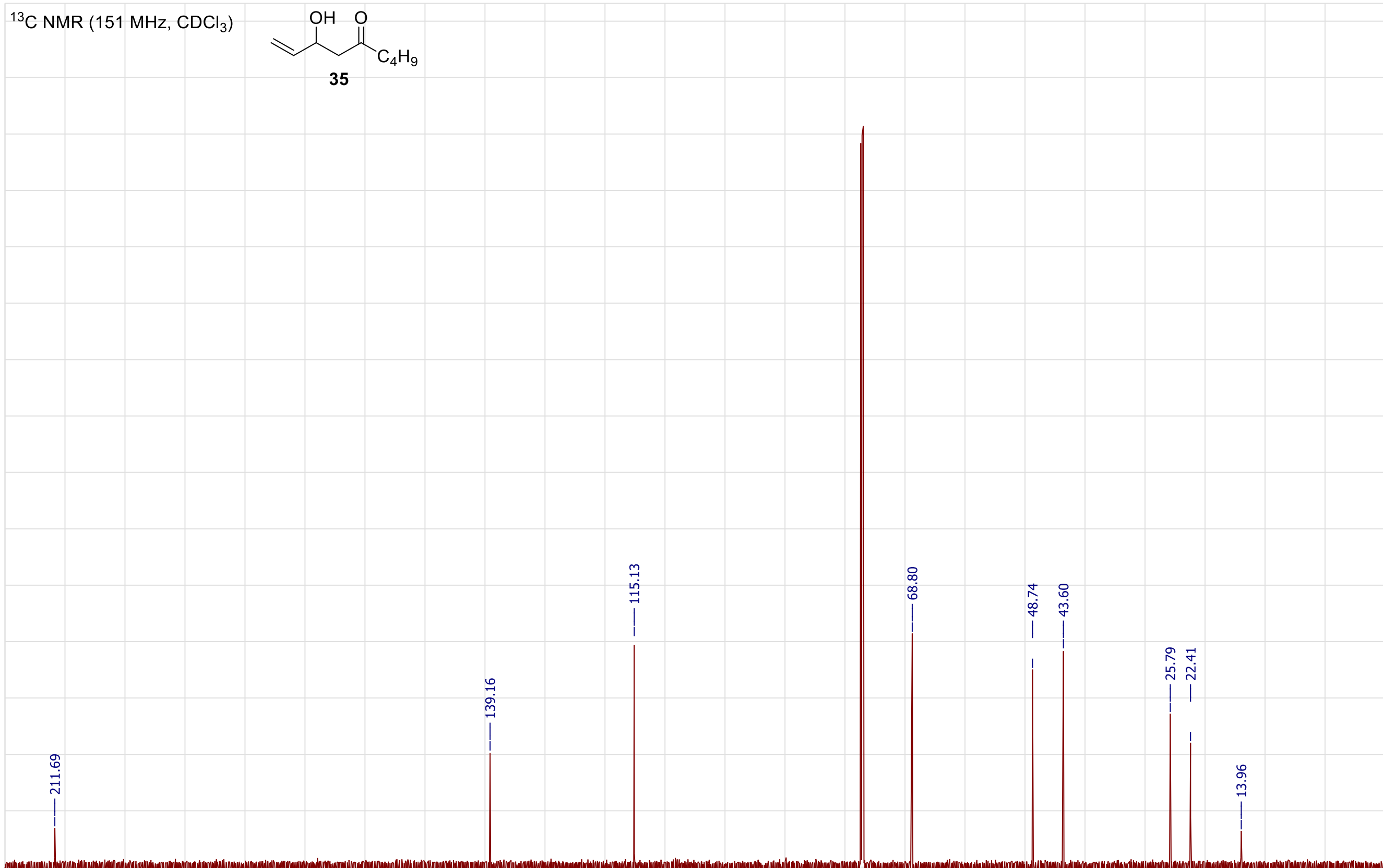

$\begin{array}{lllllllllllllllllllllll}220 & 210 & 200 & 190 & 180 & 170 & 160 & 150 & 140 & 130 & 120 & \begin{array}{c}110 \\ \mathrm{f} 1(\mathrm{ppm})\end{array} & 90 & 80 & 70 & 60 & 50 & 40 & 30 & 20 & 10 & 0 & -10\end{array}$




\section{${ }^{1} \mathrm{H} \mathrm{NMR}\left(600 \mathrm{MHz}, \mathrm{CDCl}_{3}\right)$}

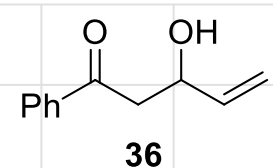

36

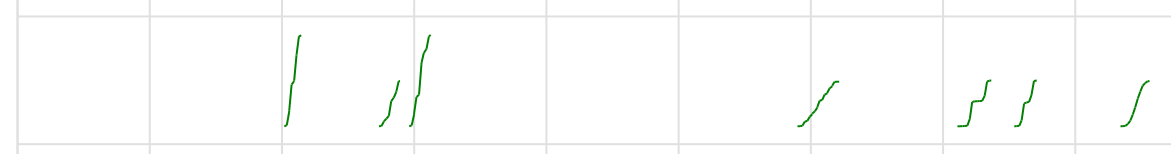

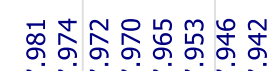

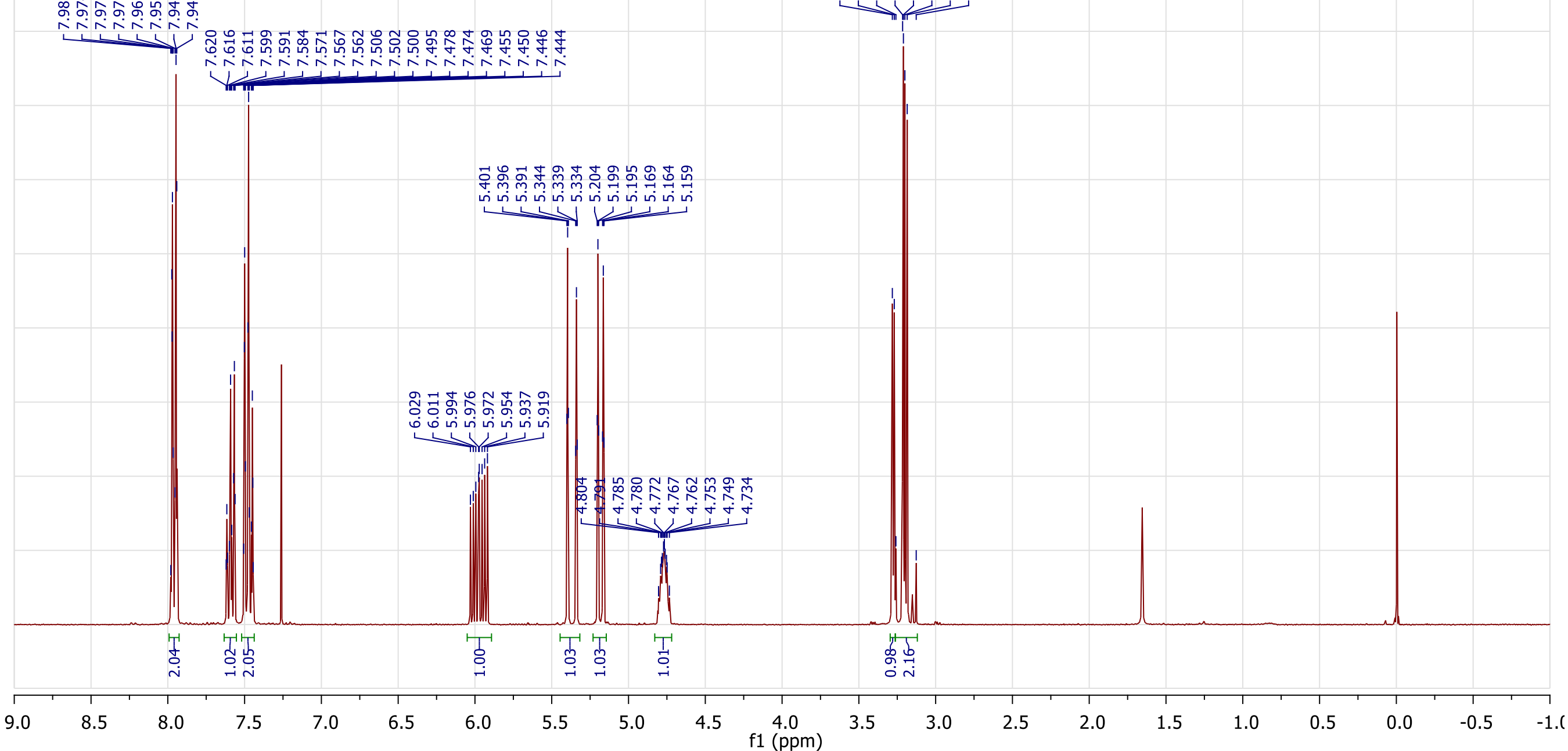



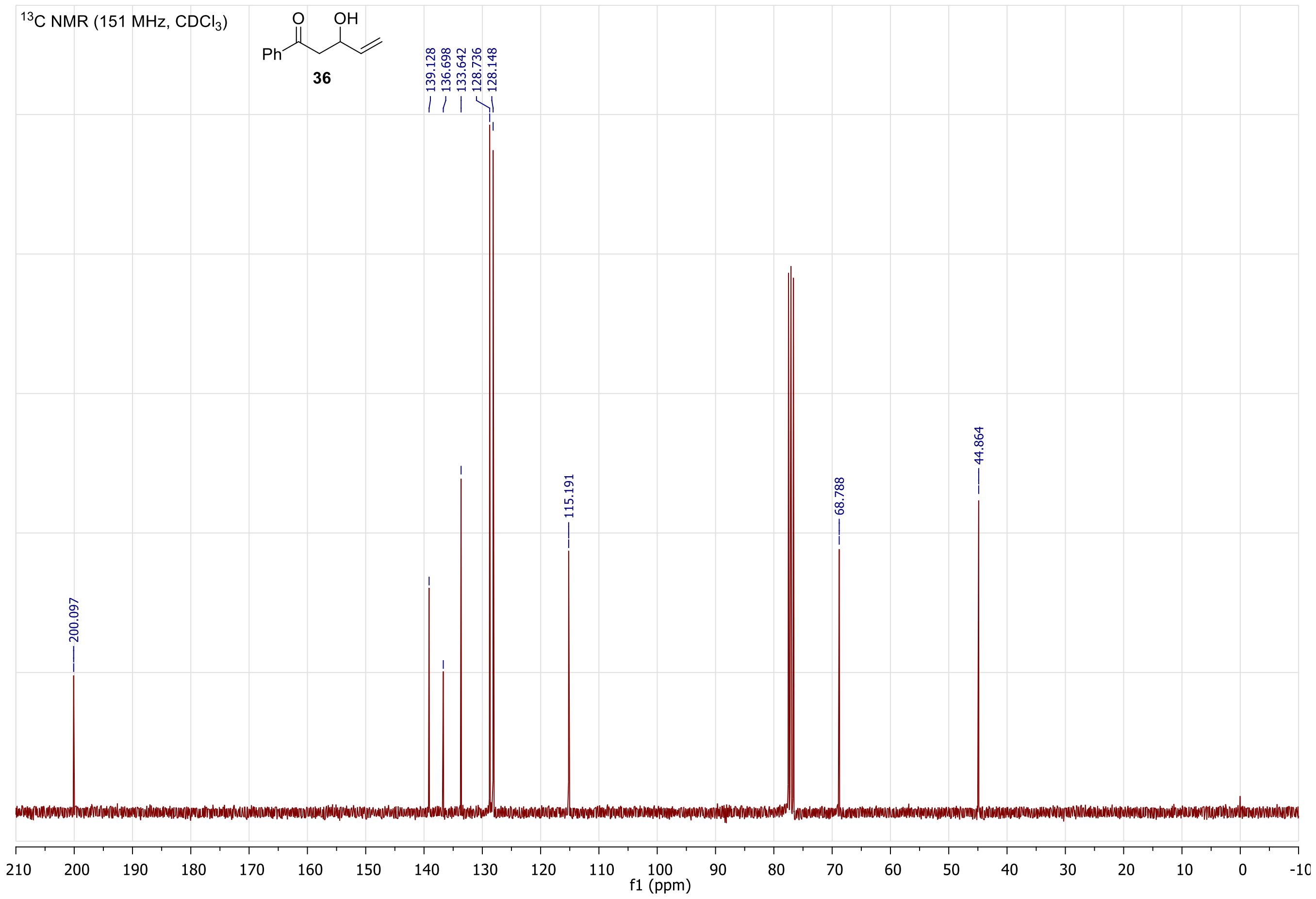

S95 